Published in final edited form as:

J Med Chem. 2019 November 14; 62(21): 9600-9617. doi:10.1021/acs.jmedchem.9b01030.

\title{
Structural Basis for Achieving GSK-3 $\beta$ Inhibition with High Potency, Selectivity, and Brain Exposure for Positron Emission Tomography Imaging and Drug Discovery
}

\author{
Vadim Bernard-Gauthier ${ }^{\star}, \dagger, \ddagger, \S$, Andrew V. Mossine ${ }^{\|}$, Ashley Knight ${ }^{\dagger, \ddagger, \perp}$, Debasis Patnaik ${ }^{\#}$, \\ Wen-Ning Zhao\#, Chialin Cheng\#, Hema S. Krishnan§, Lucius L. Xuan\#, Peter S. \\ Chindavong\#, Surya A. Reis\#, Jinshan Michael Chenף, Xia Shao", Jenelle Stauff", Janna \\ Arteaga", Phillip Sherman", Nicolas Salem ${ }^{\nabla}$, David Bonsall ${ }^{\circ}$, Brenda Amaral ${ }^{\nabla}$, Cassis \\ Varlow $^{\dagger}$, Lisa Wells ${ }^{\bigcirc}$, Laurent Martarello ${ }^{\nabla}$, Shil Patel ${ }^{\perp}$, Steven H. Liang ${ }^{\S}$, Ravi G. \\ Kurumbail ${ }^{\Uparrow}$, Stephen J. Haggarty", Peter J. H. Scott ${ }^{\|,}$, , Neil Vasdev ${ }^{\star}, \dagger, \ddagger, \S$ \\ ${ }^{\dagger}$ Azrieli Centre for Neuro-Radiochemistry, Research Imaging Centre, Centre for Addiction and \\ Mental Health, Toronto, Ontario M5T 1R8, Canada \\ ‡Department of Psychiatry/Institute of Medical Science, University of Toronto, Toronto, Ontario \\ M5T 1R8, Canada \\ §Division of Nuclear Medicine and Molecular Imaging, Massachusetts General Hospital and \\ Department of Radiology, Harvard Medical School, Boston, Massachusetts 02114, United States \\ "Division of Nuclear Medicine, Department of Radiology, The University of Michigan Medical \\ School, Ann Arbor, Michigan 48109, United States \\ ${ }^{\perp}$ Eisai AiM Institute, Boston, Massachusetts 01810, United States \\ \#Chemical Neurobiology Laboratory, Massachusetts General Hospital, Center for Genomic \\ Medicine, Departments of Neurology \& Psychiatry, Harvard Medical School, Boston, \\ Massachusetts 02114, United States \\ IPfizer Worldwide Research and Development, Groton Laboratories, Eastern Point Road, Groton, \\ Connecticut 06340, United States
}

\footnotetext{
*Corresponding Authors: vadimbgauthier@gmail.com (V.B.-G.); neil.vasdev@utoronto.ca (N.V.). Author Contributions

V.B.-G. and A.V.M. contributed equally to this work. V.B.-G., N.V., A.K., D.P., W.-N.Z., P.S.C., J.M.C., N.S., L.W., L.M., R.G.K., and S.J.H. designed research; V.B.-G., A.V.M., N.V., A.K., D.P., W.-N.Z., C.C., L.L.X, P.S.C., S.A.R., J.M.C., X.S., J.S., J.A., P.S., H.S.K., S.H.L., S.P.; N.S., L.W., and L.M. performed research; V.B.-G., N.V., N.S., L.M., R.G.K., S.J.H., and P.J.H.S. contributed new reagents/analytical tools; V.B.-G., A.V.M., N.V., A.K., D.P., W.-N.Z., C.C., P.S.C., J.M.C., S.P., N.S., C.V., L.W., L.M., R.G.K., and S.J.H. analyzed data; V.B.-G. wrote the paper.

Supporting Information

The Supporting Information is available free of charge on the ACS Publications website at DOI: 10.1021/acs.jmed-chem.9b01030. Additional figures illustrating chemistry and radiochemistry methods, HEK-293T P301S hTau cellular assay, in vitro autoradiography, kinase assays, MST assays, culturing and neural differentiation of human iPSC-derived NPCs, WNT signaling reporter assays, NPC proliferation assay with CellTiter-Glo, high-content neuronal assay for p-CRMP2 ${ }^{\mathrm{T} 514}$, pTau assay, western blot analysis, rodents and nonhuman primate PET imaging studied, neuropharmacokinetic analysis, and NMR spectra (PDF)

Molecular formula strings (XLSX)

Accession Codes

Existing PDBs 5KPK and 5K5N were used for in silico docking studies.

The authors declare no competing financial interest.
} 
${ }^{\nabla}$ Biogen, Research and Early Development Imaging, Cambridge, Massachusetts 02142, United States

OInvicro, London W12 0NN, U.K.

-The Interdepartmental Program in Medicinal Chemistry, University of Michigan, Ann Arbor, Michigan 48109, United States

\section{Abstract}

Using structure-guided design, several cell based assays, and microdosed positron emission tomography (PET) imaging, we identified a series of highly potent, selective, and brain-penetrant oxazole-4-carboxamide-based inhibitors of glycogen synthase kinase-3 (GSK-3). An isotopologue of our first-generation lead, $\left[{ }^{3} \mathrm{H}\right] \mathrm{PF}-367$, demonstrates selective and specific target engagement in vitro, irrespective of the activation state. We discovered substantial ubiquitous GSK-3-specific radioligand binding in $\mathrm{Tg} 2576$ Alzheimer's disease (AD), suggesting application for these compounds in AD diagnosis and identified $\left[{ }^{11} \mathrm{C}\right] \mathrm{OCM}-44$ as our lead GSK-3 radiotracer, with optimized brain uptake by PET imaging in nonhuman primates. GSK- $3 \beta$-isozyme selectivity was assessed to reveal OCM-51, the most potent $\left(\mathrm{IC}_{50}=0.030 \mathrm{nM}\right)$ and selective ( $>10$-fold GSK- $3 \beta /$ GSK-3 $a$ ) GSK- $3 \beta$ inhibitor known to date. Inhibition of CRMP2 ${ }^{\mathrm{T} 514}$ and tau phosphorylation, as well as favorable therapeutic window against WNT/ $\beta$-catenin signaling activation, was observed in cells.

\section{Graphical Abstract}

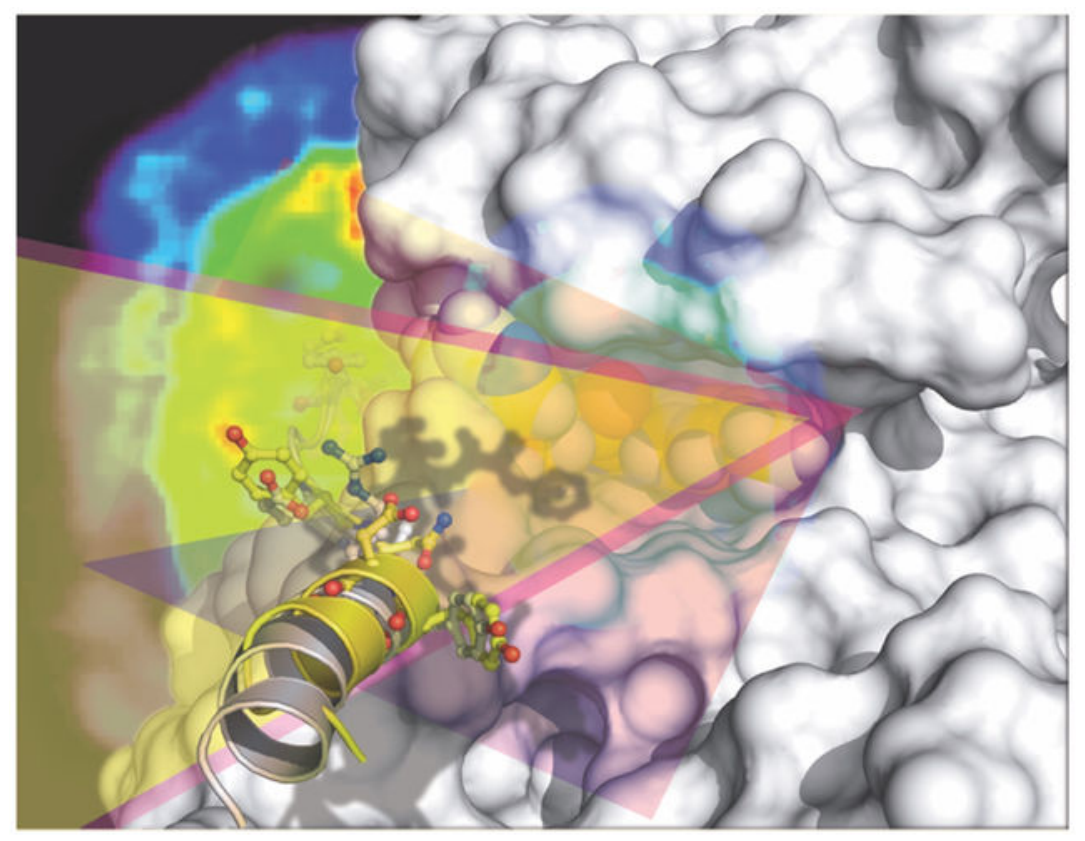

\section{INTRODUCTION}

Glycogen synthase kinase- $3 a$ and $\beta$ (GSK-3a/ $\beta$ ) are ubiquitous multifunctional serine/ threonine kinases, which regulate diverse physiological functions in peripheral organs and 
the central nervous system (CNS), ranging from metabolism and cell cycle regulation to brain development. Appropriately, balanced GSK-3 regulation and signaling is therefore central to human physiology but often compromised in diseases of diverse etiologies such as diabetes, cancers, and neurological disorders. ${ }^{1,2}$ GSK-3a/ $\beta$ are remarkably networked enzymes, with involvement in numerous pathways and over one hundred distinct putative substrates. ${ }^{3}$ Also, unusual among kinases are the fact that GSK- $3 a / \beta$ regulation occurs via inactivation while resting cells show high levels of catalytic activity. The inactivation of GSK-3 has been well-characterized and revealed primarily to proceed through N-terminus serine phosphorylation catalyzed by kinases such as AKT/PKB, p70RSK, and p90RSK (at serine 21 and serine 9 for GSK- $3 a$ and GSK-3 $\beta$, respectively). ${ }^{4,5}$ Of note, GSK-3 $a$ and GSK-3 $\beta$ present a significant kinase domain homology with one another, especially within the ATP-binding pocket (97\% identity). ${ }^{6,7}$ Congruently, GSK-3 $a / \beta$ also exhibits substantial functional overlap, although some elements of nonredundancy such as GSK-3 $\beta$-specific perinatal lethality have been characterized from paralogue-specific Gsk3 knockout model studies, which together support the notion that individual isozyme contributions should be defined carefully. ${ }^{7-9}$

Several immunohistochemical, cellular, transgenic, and preclinical studies have implicated GSK-3 deregulation as a contributive factor in neurological diseases including Alzheimer's disease (AD) and non-AD tauopathies. ${ }^{10-12}$ While in most cases, GSK-3 $\beta$ has been the central focus in these studies, both GSK-3a/ $\beta$ overexpression and inhibition/knockdown experiments have demonstrated roles in APP processing and correlated with increased or decreased amyloid- $\beta$ production, respectively. ${ }^{13,14}$ In turn, amyloid- $\beta$ injections in rat trigger APP-processing changes and pathological tau phosphorylation linked to GSK- $3 \beta$ overexpression and activation. ${ }^{15}$ The hyperphosphorylation of tau by GSK-3 $\alpha / \beta$ also suggests a central implication in neurofibrillary tangle formation, however whether an increase in GSK-3a/ $\beta$ levels or activities alone, rather than parallel changes in phosphatase activities, constitute the causative pathogenic factor in tau hyperphosphorylation and neurofibrillary tangle accumulation remains to be clarified. ${ }^{16-18}$ Both inhibition of GSK-3 in transgenic mice-overexpressing human tau and overexpressing conditional Gsk3b mice were associated with changes in hyperphosphorylated tau protein (pTau) levels tightly linked to GSK-3 levels and activity in vivo. ${ }^{19,20}$ Current evidence using human tissue suggests, as with most immunohistochemical cellular and transgenic rodent analyses, increases in total and active GSK-3 $\beta$ levels in AD compared to healthy controls. ${ }^{21-23}$ However, data gathered in human brains have been largely insufficient to substantiate in vitro preclinical findings while in vivo pharmacological intervention studies in transgenic mice as well as clinical trials aimed at validating GSK-3 in humans have been obscured by the systematic use of promiscuous inhibitors. ${ }^{24-26}$ Indeed, much of the inability to define whether GSK-3 is an actionable target in neurodegenerative diseases in vivo ensue from the lack of suitable molecular tools. To address this, highly potent, selective, and brain penetrant inhibitors of GSK-3 are needed. However, apart from rare exceptions where some of these criteria have been met, so far clinical and preclinical GSK-3 inhibitors have been both sub-optimal regarding potency or brain exposure and have displayed, despite sporadic claims of selectivity in restricted kinase subsets, poor overall kinome selectivity when systematically evaluated in large-scale screening. ${ }^{27-31}$ Moreover, only one recent report has described 
kinome-selective GSK-3 inhibitors also achieving $a / \beta$-isozyme selectivity, however, none of these isozyme-selective compounds exhibit single-digit nanomolar potencies or have been characterized in the CNS tissue. ${ }^{32}$

Herein, we used structure-guided design and microdosed positron emission tomography (PET) imaging to develop novel small-molecule inhibitors of GSK-3 achieving high potency, selectivity, and brain exposure. We describe the rational development of the novel GSK-3 $\beta$-isozyme selective inhibitor, named herein as OCM-51 (51), which to the best of our knowledge, constitutes the most potent and selective GSK-3 inhibitor identified thus far. Biochemical, biophysical, cellular, and brain tissue-based assays collectively were used to confirm highly selective target engagement. Further analysis also demonstrates differential binding profiles in $\mathrm{AD}$ transgenic mice brains versus wild-type. Together, our results describe finely characterized candidate inhibitors which can lead to the development of GSK-3 specific therapeutics as well as diagnostic GSK-3 PET molecular probes for neuroimaging.

\section{RESULTS}

\section{[ $\left.{ }^{3} \mathrm{H}\right]$ PF-367 Engages GSK-3 with High Specificity in Cells and Human Postmortem Brain.}

Previously, we reported the discovery of PF-04802367 (PF-367; 1), a potent type-I oxazole-4-carboxamide dual GSK-3a/ $\beta$ inhibitor, showing promising ADME properties combined with robust CNS/peripheral pTau and muscle pGS inhibition in vivo. ${ }^{28} \mathrm{Here}$, we first tested whether the lead compound, PF-367, displayed specific GSK-3 $\alpha / \beta$ binding in the mammalian brain tissue. We developed $\left[{ }^{3} \mathrm{H}\right] \mathbf{1}$ (Figure 1A) and observed, in rat, marmoset and human brains (frontal cortex and hippo-campus), high and nearly ubiquitous total binding in the gray matter, consistent with known GSK-3a/ $\beta$ mRNA and protein levels (Figures 1B-F and S1B,C). Highest radioligand binding was measured in the cerebral cortex and consistently more pronounced within gray matter regions compared to the white matter in all species (50-60\% difference). To confirm that our GSK-3 $\alpha / \beta$ marker provided saturable signal, homologous competition was performed on adjacent sections using unlabeled $\mathbf{1}$ at $10 \mu \mathrm{M}$ and resulted in significantly reduced binding compared to baseline experiments and overall low levels of nonsaturable binding (>87,66, and $85 \%$ reductions in rat, marmoset, and human cerebral cortices, respectively, Figure 1B,D,E). We then used two structurally unrelated and well-characterized heterologous pan-GSK-3 competitive inhibitors to determine on-target binding selectivity. Single concentration cotreatments with the advanced preclinical inhibitor CHIR99021 and the clinical lead LY2090314 both resulted in substantial decreases in tracer binding similar to unlabeled $\mathbf{1}$ incubations (Figures 1B-F and S1A). In all species and brain regions, there were no significant discrepancies in blocking effects between 1 and the heterologous probes, indicating that GSK-3a/ $\beta$ specificity could be determined using any of these inhibitors interchangeably.

To further assess our probe in a cellular context, $\left[{ }^{3} \mathrm{H}\right] \mathbf{1}$ binding in HEK293T P301S hTau cells overexpressing GSK- $3 a / \beta$, shRNA-induced GSK-3 $a / \beta$ knockdown, and nonoverexpressing HEK293T P301S hTau cells was compared (Figures 1H-J and S1F). Significantly higher binding was detected in overexpressing cells compared to GSK-3a/ $\beta$ knockdown (three-fold) and control (six-fold) while cold chase studies (where 
nonradioactive agent is administered following the radiotracer) resulted in consistent timedependent binding reductions in all cells (Figure 1H,I). Strong, dose-dependent, GSK-3 specific, and reversible binding was observed with overexpressing HEK293T P301S hTau cells when incubated with $\left[{ }^{3} \mathrm{H}\right] \mathbf{1}$ in competition (co-incubation, $85 \%$ displacement at $5 \mu \mathrm{M}$ ) and pulse chase (96\% displacement at $5 \mu \mathrm{M}$ for $5 \mathrm{~min}$ ) studies with a third heterologous inhibitor (e.g., AR79, Figure S1A,G). Further evidence of GSK-3 $a / \beta$ target engagement and selectivity on brain tissue was found when comparing tumor to normal CNS contralateral tissue binding of $\left[{ }^{3} \mathrm{H}\right] \mathbf{1}$ in rat brain sections with intracranial GS9L gliomas in vitro (>40\% binding differences, Figure S1D,E). We next performed binding saturation experiments using mouse brain homogenates which determined a GSK-3 $a / \beta$ binding site density $\left(B_{\max }\right)$ of $960.33 \pm 234 \mathrm{fmol} / \mathrm{mg}$ and an affinity in line with biochemical data $\left(K_{\mathrm{D}}=13.02 \pm 3.3\right.$ $\mathrm{nM}$ ) (Figure $1 \mathrm{G})$. Consistently throughout these cellular and whole tissue assays, the observed signals are predictably derived from GSK- $3 a$ and GSK-3 $\beta$ - as large-scale proteomic analyses have indicated only about two-to fourfold higher CNS $\mathrm{B}_{\max }$ for GSK-3 $\beta$ when compared to GSK-3 $a .{ }^{33,34}$ Together, these results also indicated that the $\mathbf{1}$ scaffold aligns suitable target engagement and selectivity properties to serve as a starting point in our lead-to-candidate optimization efforts.

\section{Development and Characterization of Selective Oxazole-4-carboxamide GSK-3 Inhibitors.}

The hit-to-lead screening campaign which led to 1 had pointed out the importance of three key fragments with regard to GSK-3 potency and selectivity: (1) the 1,2,4-triazol-1-yl group involved in a cation $-\pi$ interaction with $\operatorname{Arg} 141$; (2) a critical core oxazole-4-carboxamide moiety providing hinge domain-ligand hydrogen bond-pairing interactions around Val135/ Asp133; and (3) the 3-chloro-4-methoxyphenyl ring mainly involved in hydrophobic contacts within the deep pocket formed between the $\mathrm{N}$ - and C-lobes in the vicinity of Phe67, Lys85, and Leu132 (GSK-3 $\beta$ residues) and corresponding GSK-3 $a$ residues. ${ }^{28}$ On the basis of the aforementioned in vitro cell and tissue studies, and guided by structural data gained previously, we undertook structural refinement efforts to improve blood-brain barrier (BBB) permeation, which is suboptimal for $\mathbf{1},{ }^{28}$ and in vivo properties of our lead while retaining or improving physical and biochemical properties. ${ }^{28}$ To identify next-generation inhibitors, $>50$ optimized compounds were synthesized and underwent biochemical screening (Figure 2B, Supporting Information Materials and Methods and Tables S1-S4). In designing derivatives, emphasis was placed on the exploration of the effects of halogenation patterns, primarily fluorinations, and the modulation of the cation $-\pi$ interacting group while keeping the oxazole-4-carboxamide pharmacophore intact and minimizing changes detrimental to calculated Fu_p (unbound brain fraction) and CNS multiparameter optimization (MPO) scores (properties which are suboptimal for most known GSK-3 inhibitors, including PET probes evaluated to date, Figure 2B, Supporting Information Materials and Methods and Tables S1-S4). Our structure-activity relationship (SAR) study around the cation- $\pi$ interacting side chain amide substituents showed that 6-membered nitrogen-containing rings (pyrazine, pyridines) combined with 3-"chlorine-for-fluorine" substitutions at the 4methoxyphenyl fragment were well tolerated. We identified three inhibitors with such structural patterns displaying single-digit nanomolar potencies toward GSK-3 $\beta$ (namely, PF-618 (28), OCM-37 (37), and OCM-44 (44); $\mathrm{IC}_{50}<2.5 \mathrm{nM}$ ) in our ADPGlo screening assay (Figure 2A). Gratifyingly, these structural modifications were associated with 
profound CNS-penetration enhancements in vivo (vide infra). The high binding affinity and dual GSK-3a/ $\beta$ inhibitory nature of these analogues were confirmed in a second biochemical assay format, utilizing radiometric filtration $\left(\gamma-\left[{ }^{33} \mathrm{P}\right]\right.$-ATP kinase assay, Supporting Information Materials and Methods) (GSK-3a/ $\beta K_{\mathrm{i}}=3.3 / 4.2 \mathrm{nM}, 28$, Figure $2 \mathrm{C}, \mathrm{D})$. When assayed against a comprehensive panel of 371 kinase targets, these inhibitors displayed distinctive selectivity, inhibiting only two targets (DYRK1A and DYRK1B) beyond $\mathrm{IC}_{50}$ concentrations at $1.0 \mu \mathrm{M}$ (Figure 2F, Tables S5 and S6). While activity overlap between GSK-3 and the Down syndrome kinases DYRK1s is common across inhibitors aimed at both targets, we observed that in this case, it may be exacerbated by ligand-receptor $\pi-\pi$ interactions from a conserved DYRK1A/B Asp247/198, mimicking the guanidinium Arg 141 cation $-\pi$ interaction (Figure 2E,G-I). ${ }^{35}$ However, we found 40-170-fold selectivity for GSK-3a/ $\beta$ when measuring specific inhibitory constants even against the closest offtargets (DYRK1A/DYRK1B $K_{\mathrm{i}}=325 \mathrm{nM} / 171 \mathrm{nM}$; Figure 2C,D), well within the requirements expected for therapeutics and PET radioligand development.

\section{Oxazole-4-carboxamide Probes Engage npS9-GSK-3 $\beta$ and pS9-GSK-3 $\beta$ Irrespective of Activation States.}

Pathological changes associated with GSK- $3 \beta$ may be overexpression- or overactivationdriven, therefore it is crucial to address whether molecular probes engaging GSK- $3 \beta$ do so dependently or independently of the $S 9$ phosphorylation status in order to extrapolate valid imaging applications (S21 in GSK-3 $a$ isozyme). To elucidate possible target engagement discrepancies between npS9-GSK-3 $\beta$ (active nonphospho-S9) and pS9-GSK-3 $\beta$ (inactive phospho-S9), we developed a high-sensitivity microscale thermophoresis (MST)-based binding assay. Use of MST allows for high-precision determination of equilibrium dissociation constants by measuring temperature-induced changes in a fluorescently labeled target protein as a function of small amounts of nonfluorescent small molecules with minimal influence by ATP concentration and other such factors that impact functional cellbase assays. ${ }^{36}$ We generated catalytically inactive pS9-GSK- $3 \beta$ by treating nonphosphorylation active npS9-GSK-3 $\beta$ (Figure 3B) with AKT and ATP. When npS9GSK- $3 \beta$ was treated with AKT and high ATP concentration for $3 \mathrm{~h}$, complete loss of catalytic activity was observed, and high level of $\mathrm{pS} 9-\mathrm{GSK}-3 \beta$ was produced as determined by ADPGlo kinase and western blot analyses (Figure 3C,D). Under control conditions using ATP treatments alone, GSK-3 $\beta$ catalytic activity remained unaffected (Figure 3C,D).

Characterization by MST of the npS9-GSK- $3 \beta$ and pS9-GSK- $3 \beta$ proteins first confirmed the high binding affinities of oxazole-4-carboxamide inhibitors toward npS9-GSK-3 $\beta\left(K_{\mathrm{d}}=0.8\right.$ and $4.5 \mathrm{nM}$ for $\mathbf{1}$ and $\mathbf{4 4}$ respectively) and revealed only marginal differences versus inactive pS9-GSK-3 $\beta$ with regard to target engagement (<two-fold, Figure 3E-I). Under the same conditions, CHIR99021 GSK-3 $\beta$ binding was also largely independent of the S9 status ( $K_{\mathrm{d}}$ $=48.8$ and $28.3 \mathrm{nM}$ for pS9-GSK- $3 \beta$ and npS9-GSK-3 $\beta$, respectively, Figure S2A).

Consistent with these MST results, in silico experiments and X-ray crystal structure analyses indicated that conformational changes occurring upon N-terminal inhibitory peptide sequence binding do so remotely from the inhibitor-binding site (Figure 3A). GSK-3 $\beta$ structural remodeling following pS9 autoinhibition primarily affects the C-loop and activation segment, which are positioned distantly from the bound inhibitor and have 
negligible impact on the ATP-binding site conformations, thus having limited impact on the target engagement of type-I ATP competitive inhibitors as observed here (Figure 3A). ${ }^{37}$ On the basis of these results, we conclude that oxazole-4-carboxamide-based probes, such as $\left[{ }^{3} \mathrm{H}\right] \mathbf{1}$ and positron emitter-labeled derivatives thereof, provide a genuine assessment of total GSK-3 density irrespective of the S9 phosphorylation.

\section{Discovery of Structural Determinants To Generate Picomolar GSK-3 $\beta$-Isozyme Selective Inhibitors.}

In the course of the initial SAR study, we observed that the orientation of the 3-halogen phenyl substituents found in this series was optimal with respect to the deep hydrophobic cleft found in GSK-3 $\beta$ (Figures $2 \mathrm{~A}, \mathrm{H}$ and $\mathrm{C}, \mathrm{E}-\mathrm{G}$ ). Close inspection of GSK-3 $\beta-\mathbf{1}$ and other GSK- $3 \beta$ cocrystal structures indicated that, as recently noted by Wagner et al. 2018, the topology of this pocket is dependent on a complex backend hydrogen-bonding network involving the side chain of Asp133, the only nonconserved residue from the ATP-binding site between GSK-3 $a$ and GSK-3 $\beta$ (Glu196 in GSK-3 $a$ ). ${ }^{32}$ We observed that the hydrogen bond interactions in GSK-3 $\beta$ which bring the side chains of Asp133, Arg113, and Glu80 in close contact also directly impact proximal hydrophobic residues from $\beta$ sheets 5 and 6 (gatekeeper Leu132, Leu130 and Leu112), pulling these residues away from the ATPbinding site and forcing the opening a deep yet narrow hydrophobic pocket, wherein the paralogue GSK-3 $a$ residue Glu196 could lack the ability to secure such interactions in the first place. Finer tuning of the occupation of this pocket using subtle variation in halogen patterning, we reasoned, would allow for the possibility of achieving $\beta$-isozyme selectivity at high potency.

To assess this, we developed a subseries of derivatives leading to inhibitor $\mathbf{5 1}$, which gratifyingly provided not only 65 -fold improvement in potency compared to 44 (GSK-3 $\beta$ $\mathrm{IC}_{50}=0.031 \mathrm{nM}$ ) but also $>10$-fold selectivity for GSK-3 $\beta$ over GSK- $3 a$ (Figures 4A,C,E$\mathrm{G}$ and $\mathrm{S} 2 \mathrm{~B})$. Similar results were obtained using our confirmation $\left[{ }^{33} \mathrm{P}\right]$-radiometric binding assays $\left(K_{\mathrm{i}}=0.10 \mathrm{nM},>10\right.$-fold GSK- $3 \beta$ selectivity over GSK-3 $a$, Figure 4B,D). Increases in potency moving from 44 to 1 (via inhibitor OCM-47 (47)/49 (49)) could be attributed to both the incorporation of gradually bulky aryl halides substituents $(\mathrm{F}<\mathrm{Cl}<\mathrm{I})$ and the use of an optimized 5-fluoropyridin-3-yl cation- $\pi$ interacting moiety (Figure 4A,D). However, the $\beta$-isozyme selectivity occurred specifically with the iodoaryl substitution (Figure $4 \mathrm{E}-\mathrm{G}$ ). We confirmed this effect in a similar subseries wherein the cation $-\pi$ interacting fragment was kept untouched ([PF-618 (28), OCM-45 (45) and OCM-32 (32) Figure 4A,D, Tables S3 and S4]. As for previous compounds of this series, these inhibitors displayed exquisite selectivity in broad kinome coverages (Figure 4I). Inhibitor $\mathbf{5 1}$ demonstrated improved selectivity even compared to previous inhibitors from this series (vide supra), with $\geq 10000$-fold selectivity against all 371 kinases tested including the DYRK family. Further analysis of the potent inhibitor 49 as a representative example of this series in Kinativ proteomic screening using mouse brain lysate revealed extensive GSK-3a/ $\beta$ kinase engagement without any detectable off-target interactions ( $>215$ kinases, Figure $4 \mathrm{H})$. Notably, the Kinativ profiling used here included $>40$ CNS-enriched kinases not initially covered in recombinant enzyme panels. We obtained dissociation constants from MST analysis in line with biochemical data and showed, as previously noted, no quantifiable affinity differences between npS9-GSK-3 $\beta$ and 
pS9-GSK-3 $\beta$ enzymes (51 $K_{\mathrm{d}}=0.39 \mathrm{nM}$ and $K_{\mathrm{d}}=0.49 \mathrm{nM}$ for npS9-GSK-3 $\beta$ and pS9GSK-3 $\beta$, respectively; Figures $4 \mathrm{~L}, \mathrm{M}$ and S3A). Employing a high concentration cutoff of 10 $\mu \mathrm{M}$, we also found negligible off-target binding for the representative inhibitor $\mathbf{5 1}$ in a CNSrelated panel, with only 5 of 80 proteins (including receptors, ion channels and transporters), exhibiting $>70 \%$ inhibition at this concentration (Figure $4 \mathrm{~J}$ ). Follow-up inhibitory constant measurements revealed $>1000-20000$-fold gaps in favor of GSK-3 $\beta$ in all cases providing further proof of selectivity (Figure $4 \mathrm{~K}$ ). In addition, all representative compounds tested (28, 44, and 51) display low $\mathrm{hERG}$ inhibition $\left(\mathrm{EC}_{50}=1.7-62.6 \mu \mathrm{M}\right)$ and correspondingly significant in vitro margins with regard to GSK-3 $\beta$ inhibition (8320-56667-fold, Figure $\mathrm{S} 2 \mathrm{C})$.

\section{Bioactivities of the Oxazole-4-carboxamide Inhibitors in Cells.}

We next explored the effect of oxazole-4-carboxamide inhibitors on endogenous cellular GSK- $3 \beta$ signaling. To define the cellular effect on pTau, we used high-content imaging of human cortical-like glutamatergic neurons differentiated from induced pluripotent stem cells through induced expression of Ngn2 transgene. ${ }^{18,38}$ In agreement with high biochemical potency and cellular target engagement, we found that the two novel representative compounds tested (28 and 51) caused dose-dependent decreases in pTau levels with efficacy similar to 1 (Figures 5A-D and S3C). We employed as the negative control the low potency, trifluoromethoxy-bearing structural analogue OCM-29 (29; $\mathrm{IC}_{50}=4.1 \mu \mathrm{M}$, Figure S3A) and observed no appreciable decrease in pTau levels even with doses up to $30 \mu \mathrm{M}$ (Figure 5D). We further evaluated the oxazole-4-carboxamide inhibitors in another neuronal assay, in which the phosphorylation of collapsin response mediator protein 2 (CRMP2) at Thr-514 (pCRMP2 ${ }^{\mathrm{T} 514}$ ) is measured using immunostaining, following $24 \mathrm{~h}$ treatment with test compounds. ${ }^{39}$ CRMP2 is a direct protein substrate of GSK-3 $\beta$, not GSK-3 $a$, and important for diverse neuronal functions. ${ }^{40,41}$ Compounds were tested at six doses from $20 \mu \mathrm{M}$ with 2fold dilution. Robust dose-dependent effect with $\mathrm{EC}_{50}$ ranging from 0.5 to $1.5 \mu \mathrm{M}$ was detected for all active compounds, as well as the CHIR99021 control (Figures 5E-I and $\mathrm{S} 3 \mathrm{E}, \mathrm{H})$. In contrast, the low potency, negative control 29 failed to elicit detectable pCRMP2 $^{\mathrm{T} 514}$ reductions even at the highest concentration tested $(20 \mu \mathrm{M}$, Figure $5 \mathrm{H})$.

Given the role of GSK-3 in WNT/ $\beta$-catenin signaling, we also conducted Wnt-signaling gene transcription luciferase reporter assay (Figure $5 \mathrm{~N}$ ). ${ }^{43}$ Relative to pTau and pCRMP2 ${ }^{\mathrm{T} 514}$ inhibition, significantly higher concentrations of active oxazole-4carboxamide inhibitors were required to elicit gene transcription through the WNT/ $\beta$-catenin pathway (>10-25-fold selectivity for pCRMP2 $2^{\mathrm{T} 514}$ and 3-8-fold selectivity for pTau). Although $10 \mu \mathrm{M}$ CHIR99021 was sufficient to induce 10-fold changes in reporter induction (Figure S3D), oxazole-4-carboxamide inhibitor treatment only triggered two- to threefold changes under the same conditions. GSK-3 signaling is also essential for coordinating the proliferation and differentiation of progenitor cells during brain development. ${ }^{2,42}$ We used human neural progenitor cells (NPCs) and a proliferation assay to evaluate the proneurogenic potential of these GSK-3 inhibitors. In line with previous results, effect on cell proliferation was, however, minor and observed only at micromolar concentrations for all active inhibitors (Figures 5K,N and S3I,L). Together, these data indicate that these oxazole-4-carboxamide inhibitors can access endogenous GSK-3 in human neuronal cells 
and modulate functions of various GSK-3 complexes with differential efficacies, delineating a potential therapeutic window wherein the inhibition of GSK-3 $\beta$ leads to the modulation of key neuronal molecular targets while sparing putative mechanism-based $\beta$-catenin-driven effects.

\section{Oxazole-4-carboxamide Probes Display Increased Specific Binding in Tg2576 Mice Brains in Vitro and Robust BBB Permeation in Vivo.}

Given the implication of GSK-3a/ $\beta$ in APP processing and AD pathophysiology, we sought to determine in vitro GSK-3a/ $\beta$-targeted radioligand binding in the brain tissue from transgenic mice with significant amyloid plaque burden. Remarkably, comparison between Tg2576 mice (15 month) and old wild-type animals (21 and 28 months) revealed significant differences in $\left[{ }^{3} \mathrm{H}\right] \mathbf{1}$ total and specific binding in the brain tissue (Figure 6A). As previously, we carried out triple-blocking experiments (10 $\mu \mathrm{M}$ of 1, CHIR99021, or LY2090314) to confirm specificity and selectivity (80-91\% blocking) in these tissues (Figure 6A). Although we observed a suitable total and specific binding level correlation between old control mice and other normal brain tissue from rats, marmosets, and humans (vide supra), we discovered important increases in total/specific binding in $\mathrm{Tg} 2576$ mice compared to 21-month controls. Binding changes were drastic and most pronounced in regions known to exhibit high amyloid plaque deposition such as the cortex (17-fold increase) while binding in regions which present with lower or negligible plaque burden such as the hippocampus (six-fold increase) or the caudate putamen (four-fold increase), respectively, were significantly less affected (Figure 6C) ${ }^{44,45}$ In addition, regional distribution aligned well with previously characterized ubiquitous tracer binding in the brains of all other species (Ctx $\geq \mathrm{HP} \geq \mathrm{CB} \geq$ $\mathrm{CPu}>\mathrm{WM})$ (Figure 1).

With the dual goals of identifying putative GSK-3-targeted therapeutics for CNS diseases and defining structural basis to achieve PET neuroimaging for this target, we coupled the initial SAR component of this study with iterative micro-dosing rodent PET imaging experiments to gain insight into structural factors associated with BBB permeation. As referred to previously (vide supra), this led to the early realization that six-membered nitrogen-containing replacements of the triazole ring found in $\mathbf{1}$, while upholding or improving other properties from our lead, triggered significant increases in brain exposure. Among selected inhibitors $\left(\left[{ }^{11} \mathrm{C}\right] \mathrm{PF}-627(\mathbf{2 0}),\left[{ }^{11} \mathrm{C}\right] \mathbf{2 8},\left[{ }^{11} \mathrm{C}\right] \mathbf{3 7},\left[{ }^{11} \mathrm{C}\right] \mathbf{4 4}\right.$, and $\left[{ }^{11} \mathrm{C}\right] \mathbf{5 1}$, Supporting Information Materials and Methods Scheme S5 and Figures S5-S21), uptake of radioactivity in the rat brain following microdosed peripheral injection (total dose $=0.19$ $1.75 \mu$ i.v.) was variable but in all cases non-negligible with whole brain $\mathrm{SUV}_{\text {peak }}$ ranging from0.84 to 3.08 followed by rapid washout (Figure S4A). We observed the highest BBB permeation with $\left[{ }^{11} \mathrm{C}\right] \mathbf{2 8}$ and $\left[{ }^{11} \mathrm{C}\right] \mathbf{4 4}$ while the lowest radioactivity brain uptake was seen with the most potent inhibitor, $\left[{ }^{11} \mathrm{C}\right] \mathbf{5 1}$, which may indicate substantial GSK-3 binding in the periphery prior to reaching the CNS. Alternatively, this may be because of a faster clearance from the blood. Consistently, regional brain radioactivity following administration was largely homogeneous as observed in vitro. Next, to measure brain kinetics and determine brain exposure in higher species as a mean to assess the potential of our inhibitors as therapeutics and radioligands, we performed micro-dosed PET imaging experiments in nonhuman primates (one female rhesus macaque, total dose $\leq 1.1 \mu \mathrm{g}$ i.v.). The selected 
radiolabeled inhibitors, $\left[{ }^{11} \mathrm{C}\right] \mathbf{2 8},\left[{ }^{11} \mathrm{C}\right] \mathbf{3 7}$, and $\left[{ }^{11} \mathrm{C}\right] \mathbf{4 4}$, displayed in rhesus monkey both significant brain exposure with a $\mathrm{SUV}_{\text {peak }}$ of 3.0-4.0 SUV and favorable brain kinetic for imaging and CNS-targeted therapy following injection (Figures 2B,D and S4B,C).

Radioactivity uptake was equally high in all gray matter regions but lower in the white matter. These results are in contrast with the in vivo profile of $\left[{ }^{11} \mathrm{C}\right] \mathbf{1}$, previously reported and retested here, which demonstrate only low brain uptake in rhesus macaques (Figure $6 \mathrm{~B}, \mathrm{E}) .{ }^{28}$ Finally, we conducted neuropharmacokinetic studies with our lead $\mathbf{4 4}$ to assess brain exposure over time. Conforming with microdosing data, we noted rapid $\left(T_{\max }=1.0 \mathrm{~h}\right)$ excellent brain exposure (unbound $C_{\max }=941 \mathrm{nM}$ ), following single oral administration in mice $(50 \mathrm{mg} / \mathrm{kg}$ p.o.; Figure $6 \mathrm{~F})$. The unbound brain-to-plasma ratio $\left(K_{\mathrm{p}, \mathrm{uu}}\right.$, unbound $\mathrm{AUC}_{\text {last }}$ ratios) was 1.06 and largely consistent between animals, indicating continued distribution equilibrium between brain and blood compartments and no efflux liability (Figure 6F). No clinical sign following oral administration of $\mathbf{4 4}$ at therapeutic dose in mice or micro-dosing in nonhuman primates for imaging was observed, and both procedures were well tolerated.

\section{DISCUSSION AND CONCLUSIONS}

Modulating molecular targets efficiently and with high accuracy, particularly in the brain, where the BBB severely limits the capacity of peripherally administered small molecules to achieve target engagement, is a significant challenge in CNS drug discovery. To date, the roles of GSK-3 $3 a / \beta$ in neurodevelopmental and neurodegenerative diseases have been difficult to probe pharmacologically, owing to the complex nature of GSK-3a/ $\beta$-mediated cellular pathways and the lack of suitable inhibitors. The data presented here describe brainpenetrant selective inhibitors, which potently engage and inhibit GSK-3 in the mammalian brain tissue, including human brains, and modulate GSK-3-regulated neuronal pathways in cells. We also define the structural underpinning for achieving picomolar-selective GSK- $3 \beta$ inhibition as well as $\beta$-isozymespecificity, exploiting discrete topological variations from the deep ATP-binding site of GSK-3 isozymes as previously noted by Wagner et al. 2018. ${ }^{32}$ While exhibiting GSK- $3 \beta$ selectivity in ranges described for the few previously known GSK-3 isozyme selective inhibitors, the compounds described here do so at over two orders of magnitude higher potencies - which may be crucial in achieving a favorable therapeutic window for this target. ${ }^{26,32}$ Whether the maintenance of about the same fold-differences in isozyme selectivity observed here with double-digit picomolar versus previously described double-digit nanomolar potency inhibitors represents an intrinsic limitation ensuing from GSK-3 $a / \beta$ binding sites dissimilarities, or isozyme selectivity can be improved further, will require further detailed SAR work based on the results presented here. Previous studies have shown that deletion in embryonic stem cells of GSK-3 $a$ or GSK-3 $\beta$ do not individually alter $\beta$-catenin/TCF-mediated transcription, and that isoform-selective pharmacological inhibition of GSK-3 $a$ in acute myeloid leukemia cells prevents $\mathrm{Wnt} / \beta$-catenin changes at specific concentrations where dual inhibition lacks such ability—thus providing strong rationale for isozyme-selective inhibition in mitigating putative mechanism-based toxicity driven by $\beta$ catenin translocation. ${ }^{9,32}$ In the current study, the lack of dual inhibitors equipotent to $\mathbf{5 1}$ (or 32) has impeded the determination of such effect directly. It is important to note however, that inhibitors $\mathbf{5 1}$ and $\mathbf{3 2}$ only trigger $\mathrm{Wnt} / \beta$-catenin transcriptional changes at higher 
concentrations than dual and drastically lower potency controls (1 and CHIR99021). This suggests that the GSK-3 $\beta$-isozyme selective inhibitors identified here may in fact cause similar shifts wherein GSK-3 $\beta$ inhibition is achieved while sparing $\mathrm{Wnt} / \beta$-catenin. Irrespective of this effect, we note for all inhibitors identified here, a significant and favorable gap between the doses required to trigger $\mathrm{Wnt} / \beta$-catenin signaling changes and modulate $\mathrm{p}-\mathrm{CRMP} 2^{\mathrm{T} 514}$ and pTau levels.

Kinase-targeted in vivo noninvasive neuroimaging to explore intractable neurodegenerative and psychiatric conditions constitutes an unmet objective despite tremendous progress in the therapeutic and imaging use of kinase inhibitors outside the CNS. ${ }^{46,47}$ Our preclinical data demonstrate that radiolabeled oxazole-4-carboxamide inhibitors such as $\left[{ }^{11} \mathrm{C}\right] 28$ and $\left[{ }^{11} \mathrm{C}\right] \mathbf{4 4}$ align suitable physicochemical parameters, kinetics, and in vitro/in vivo properties to serve as leads to achieve GSK-3 PET imaging. ${ }^{48}$ Our analysis of GSK-3 $a / \beta$ brain density further supports the view that this target can be imaged using PET. Findings indicating that oxazole-4-carboxamide probes provide total GSK-3 density readouts and display severe binding increases in AD mice compared to wild-type animals also provide a basis for studying the potential of these probes to measure changes in GSK-3 expression in neurodegeneration. A possible caveat pertaining to the characterization of the activationindependent binding mode of the (radio)ligands described here is the report of an alternative p38 MAPK-mediated GSK-3 $\beta$ inactivation phosphorylation at the $\mathrm{S} 389 \mathrm{C}$ terminus residue. ${ }^{48}$ The relevance of this inactivation pathway has, however, only been characterized in mice and may not be relevant in human. Based on the results presented here, detailed PET quantification studies are currently underway. The major finding of this work is the discovery of orally available inhibitors with unique pharmacological profiles showing robust GSK-3 target engagement in the cell and tissue as well as high brain exposure, which warrants future intervention and imaging investigations.

\section{EXPERIMENTAL SECTION}

\section{Chemical Synthesis.}

Compound PF-04802367 ( $\mathbf{9 8 \%} \%$ ) (hereafter called 1), CHIR99021 ( $\mathbf{9 8 \%} \%$ ), and LY2090314 ( $₫ 98 \%$ ) were purchased from Millipore Sigma. Alternatively, 1 was synthesized as described previously. ${ }^{28}$ Detailed synthetic procedures for all inhibitors are provided in Schemes S1-S3 and Supporting Information Materials and Methods. Briefly, the lead inhibitor (target compound), 5-(3-fluoro-4-methoxyphenyl)- $N$-(3-(pyridin-3-yl)propyl)oxazole-4carboxamide (44, OCM-44), was synthesized as follows: the title compound was synthesized from 5-(3-fluoro-4-methoxyphenyl)-oxazole-4-carboxylic acid $(0.2 \mathrm{~g}, 0.84 \mathrm{mmol})$ and 3(pyridin-3-yl)propan-1-amine (0.137 g, $1.00 \mathrm{mmol})$ to give $44(0.145 \mathrm{~g}, 48 \%)$ as an offwhite solid. LCMS (ESI): $\mathrm{m} / z=356.10[\mathrm{M}+\mathrm{H}]^{+}$. HRMS (ESI $\left.{ }^{+}\right): \mathrm{m} / z$ calcd for $\mathrm{C}_{19} \mathrm{H}_{19} \mathrm{FN}_{3} \mathrm{O}_{3}{ }^{+}(\mathrm{M}+\mathrm{H})^{+}, 356.1410$; found, 356.1402. ${ }^{1} \mathrm{H}$ NMR (400 MHz, DMSO- $\left.d_{6}\right): \delta$ $8.55(\mathrm{~s}, 1 \mathrm{H}), 8.49$ (t, $J=5.60 \mathrm{~Hz}, 1 \mathrm{H}), 8.45(\mathrm{~d}, J=2.00 \mathrm{~Hz}, 1 \mathrm{H}), 8.39$ (dd, $J=1.40,4.80 \mathrm{~Hz}$, $1 \mathrm{H}), 8.35$ (dd, $J=2.20,13.20 \mathrm{~Hz}, 1 \mathrm{H}), 7.97$ (d, $J=8.40 \mathrm{~Hz}, 1 \mathrm{H}), 7.66-7.64(\mathrm{~m}, 1 \mathrm{H}), 7.32-$ $7.28(\mathrm{~m}, 2 \mathrm{H}), 3.91(\mathrm{~s}, 3 \mathrm{H}), 3.34-3.26(\mathrm{~m}, 2 \mathrm{H}), 2.63(\mathrm{t}, J=7.80 \mathrm{~Hz}, 2 \mathrm{H}), 1.84$ (quin, $J=7.34$ $\mathrm{Hz}, 2 \mathrm{H}) .{ }^{13} \mathrm{C}$ NMR (100 MHz, DMSO- $\left.d_{6}\right): \delta 161.09,160.65,150.78$ (d, $J=241.3 \mathrm{~Hz}$ ), 149.80, 149.74, 148.92, 148.30 (d, $J=10.6 \mathrm{~Hz}), 136.38,128.31,124.44(\mathrm{~d}, J=3 \mathrm{~Hz})$, 
122.67, 121.15, 119.68 (d, $J=8.1 \mathrm{~Hz}), 115.49$ (d, $J=21.5 \mathrm{~Hz}), 113.64,56.08,38.20,30.38$, 29.58. Purity: HPLC $t_{\mathrm{R}}=5.55 \mathrm{~min}$, HPLC purity $>95.4 \%$.

All final compounds were determined to have purity > 95\% by HPLC. A X-select CSH C18 $(4.6 \times 150 \mathrm{~mm} 3.5 \mu \mathrm{m})$ HPLC column was used to determine the chemical purity. The radiosynthesis quality control was performed on a Phenomenex Luna C18, $5 \mathrm{~mm}, 4.6 \times 150$ $\mathrm{mm}$ column. Liquid chromatography mass spectroscopy utilized a X-select CSH C18 $(3.0 \times$ $50 \mathrm{~mm} 2.5 \mu \mathrm{m})$ column.

\section{General Procedure for Synthesis of Inhibitors 6-37, 39-42, and 44-51 (OCM-6-OCM-37, OCM-39-OCM-42, and OCM-44-OCM-51).}

To a mixture of an appropriate carboxylic acid (74-84, 1 equiv), the corresponding primary amine (1.2 equiv) and 1-[bis(dimethylamino)methylene]- $1 H-1,2,3$-triazolo[4,5-b]pyridinium 3-oxide hexafluorophosphate (1.2 equiv) in dimethylformamide (DMF; 10 vol) were added triethylamine (5 equiv) in dropwise manner. The reaction mixture was stirred at room temperature for $2 \mathrm{~h}$. The progress of the reaction was monitored by thin-layer chromatography (50\% EtOAc in hexane). After completion, the reaction mass was taken into water and extracted with EtOAc, combined organic extracts were dried over anhydrous sodium sulfate, filtered, and concentrated in vacuum to get crude mass. Unless otherwise noted, purification by reverse phase column chromatography using acetonitrile-water as the mobile phase was used to obtain purified final products, which were then triturated with pentane, filtered, and dried under vacuum to afford 6-37, 39-42, and 44-51 (OCM-6OCM-37, OCM-39-OCM-42 and OCM-44-OCM-51).

\section{N-(3-(1H-1,2,4-Triazol-1-yl)propyl)-5-(3,4-difluorophenyl)-oxazole-4-} carboxamide (6, OCM-6).-The title compound was synthesized from 5-(3,4difluorophenyl)oxazole-4-carboxylic acid $(0.15 \mathrm{~g}, 0.66 \mathrm{mmol})$ and 3-(1 $H$-1,2,4-triazol-1yl)propan-1-amine $(0.10 \mathrm{~g}, 0.80 \mathrm{mmol})$ to give $6(0.12 \mathrm{~g}, 51 \%)$ as an off-white solid. LCMS (ESI): $\mathrm{m} / z=334.10[\mathrm{M}+\mathrm{H}]^{+}$. HRMS $\left(\mathrm{ESI}^{+}\right): \mathrm{m} / \mathrm{z}$ calcd for $\mathrm{C}_{15} \mathrm{H}_{14} \mathrm{~F}_{2} \mathrm{~N}_{5} \mathrm{O}_{2}{ }^{+}(\mathrm{M}+\mathrm{H})^{+}$, 334.1116; found, 334.1112. ${ }^{1} \mathrm{H}$ NMR (400 MHz, DMSO- $\left.d_{6}\right): \delta 8.66-8.64(\mathrm{~m}, 2 \mathrm{H}), 8.56-$ $8.50(\mathrm{~m}, 2 \mathrm{H}), 8.04-8.02(\mathrm{~m}, 1 \mathrm{H}), 7.96(\mathrm{~s}, 1 \mathrm{H}), 7.63-7.56(\mathrm{~m}, 1 \mathrm{H}), 4.22(\mathrm{t}, J=6.80 \mathrm{~Hz}, 2 \mathrm{H})$, 3.27 (q, $J=6.40 \mathrm{~Hz}, 2 \mathrm{H}$ ), 2.04 (quin, $J=6.80 \mathrm{~Hz}, 2 \mathrm{H}$ ).

${ }^{13} \mathrm{C}$ NMR (100 MHz, DMSO- $\left.d_{6}\right): \delta 160.62,151.37,150.43(\mathrm{~m}), 148.96(\mathrm{~m}), 148.76(\mathrm{~m})$, $147.83(\mathrm{~m}), 144.05,129.36,124.93(\mathrm{~m}), 124.3(\mathrm{dd}, J=11$ and $3.48 \mathrm{~Hz}), 117.83$ (d, $J=17.3$ $\mathrm{Hz}), 117.27(\mathrm{~d}, J=20.6 \mathrm{~Hz}), 46.52,36.00,29.39$. Purity: HPLC $t_{\mathrm{R}}=7.17 \mathrm{~min}$, HPLC purity $>99.7 \%$.

\section{N-(3-(1H-1,2,4-Triazol-1-yl)propyl)-5-(perfluorophenyl)oxazole-4-carboxamide} (7, OCM-7).-The title compound was synthesized from 5-(perfluorophenyl)oxazole-4carboxylic acid $(0.4 \mathrm{~g}, 1.43 \mathrm{mmol})$ and 3-(1 H-1,2,4-triazol-1-yl)propan-1-amine (0.108 g, $0.85 \mathrm{mmol})$ to give $7(0.06 \mathrm{~g}, 11 \%)$ as an pink-colored solid after SFC purification. LCMS (ESI): $\mathrm{m} / \mathrm{z}=388.4[\mathrm{M}+\mathrm{H}]^{+}$. HRMS $\left(\mathrm{ESI}^{+}\right): \mathrm{m} / z$ calcd for $\mathrm{C}_{15} \mathrm{H}_{11} \mathrm{~F}_{5} \mathrm{~N}_{5} \mathrm{O}_{2}{ }^{+}(\mathrm{M}+\mathrm{H})^{+}$, 388.0833; found, 388.0825. ${ }^{1} \mathrm{H}$ NMR (400 MHz, chloroform- $d$ ): $\delta 8.14(\mathrm{~s}, 1 \mathrm{H}), 8.05(\mathrm{~s}, 1 \mathrm{H})$, $7.96(\mathrm{~s}, 1 \mathrm{H}), 7.34(\mathrm{br} \mathrm{s}, 1 \mathrm{H}), 4.28(\mathrm{t}, J=6.40 \mathrm{~Hz}, 2 \mathrm{H}$ ), 3.47 (q, $J=6.8 \mathrm{~Hz}, 2 \mathrm{H}$ ), 2.20 (quin, $J$ $=6.80 \mathrm{~Hz}, 2 \mathrm{H})$. 
${ }^{13} \mathrm{C}$ NMR not assigned. Purity: HPLC $t_{\mathrm{R}}=7.21 \mathrm{~min}$, HPLC purity $>96.5 \%$.

N-(3-(1H-1,2,4-Triazol-1-yl)propyl)-5-(3,4,5-trifluorophenyl)-oxazole-4carboxamide (8, OCM-8).- The title compound was synthesized from 5-(3,4,5trifluorophenyl)oxazole-4-carboxylic acid $(0.1 \mathrm{~g}, 0.41 \mathrm{mmol})$ and 3-(1 H-1,2,4-triazol-1yl)propan-1-amine $(0.06 \mathrm{~g}, 0.48 \mathrm{mmol})$ to give $\mathbf{8}(0.058 \mathrm{~g}, 41 \%)$ as an off-white solid. LCMS (ESI): $m / z=352.10[\mathrm{M}+\mathrm{H}]^{+}$. HRMS $\left(\mathrm{ESI}^{+}\right): \mathrm{m} / z$ calcd for $\mathrm{C}_{15} \mathrm{H}_{13} \mathrm{~F}_{3} \mathrm{~N}_{5} \mathrm{O}_{2}{ }^{+}(\mathrm{M}+$ $\mathrm{H})^{+}, 352.1021$; found, 352.1020. ${ }^{1} \mathrm{H}$ NMR $(400 \mathrm{MHz}$, chloroform- $d$ ): $\delta 8.19-8.14(\mathrm{~m}, 3 \mathrm{H})$, 7.95 (s, 1H), 7.85 (s, 1H), 7.53 (br s, 1H), 4.28 (t, $J=6.40 \mathrm{~Hz}, 2 \mathrm{H}), 3.49$ (q, $J=6.40 \mathrm{~Hz}$, 2H), 2.21 (quin, $J=6.80 \mathrm{~Hz}, 2 \mathrm{H}$ ).

${ }^{13} \mathrm{C}$ NMR (100 MHz, $\left.\mathrm{CDCl}_{3}\right): \delta 160.96,152.18(\mathrm{~m}), 149.75(\mathrm{~m}), 148.23(\mathrm{~m}), 143.29$, 141.96, 139.48 (d, $J=15.7 \mathrm{~Hz}), 129.65,122.62(\mathrm{~m}), 112.69$ (m), 47.06, 36.35, 29.92. Purity: HPLC $t_{\mathrm{R}}=7.55 \mathrm{~min}$, HPLC purity $>99.6 \%$.

N-(3-(1H-1,2,4-Triazol-1-yl)propyl)-5-(3-fluoro-4(trifluoromethoxy)phenyl)oxazole-4-carboxamide (9, OCM-9).-The title compound was synthesized from 5-(3-fluoro-4-(trifluoromethoxy)phenyl)oxazole-4carboxylic acid $(0.15 \mathrm{~g}, 0.51 \mathrm{mmol})$ and 3-(1 H-1,2,4-triazol-1-yl)propan-1-amine $(0.09 \mathrm{~g}$, $0.61 \mathrm{mmol})$ to give $9(0.090 \mathrm{~g}, 44 \%)$ as an off-white solid. LCMS (ESI): $\mathrm{m} / z=400.15[\mathrm{M}+$ $\mathrm{H}]^{+}$. HRMS $\left(\mathrm{ESI}^{+}\right): \mathrm{m} / \mathrm{z}$ calcd for $\mathrm{C}_{16} \mathrm{H}_{14} \mathrm{~F}_{4} \mathrm{~N}_{5} \mathrm{O}_{3}{ }^{+}(\mathrm{M}+\mathrm{H})^{+}$, 400.1033; found, 400.1026. ${ }^{1} \mathrm{H}$ NMR (400 MHz, DMSO- $d_{6}$ ): $\delta 8.69$ (br s, 2H), 8.54 (br s, 2H), 8.11 (br s, 1H), 7.97 (br s, 1H), 7.74 (br s, 1H), 4.23 (br s, 2H), 3.30-3.21 (m, 2H), 2.05 (br s, 2H).

${ }^{13} \mathrm{C}$ NMR not assigned. Purity: HPLC $t_{\mathrm{R}}=8.05 \mathrm{~min}$, HPLC purity $>98.2 \%$.

\section{$\mathrm{N}$-(3-(1H-1,2,4-Triazol-1-yl)propyl)-5-(3-fluoro-4-} (trifluoromethyl)phenyl)oxazole-4-carboxamide (10, OCM-10).-The title compound was synthesized from 5-(3-fluoro-4-(trifluoromethyl)phenyl)oxazole-4carboxylic acid $(0.2 \mathrm{~g}, 0.73 \mathrm{mmol})$ and 3-(1H-1,2,4-triazol-1-yl)propan-1-amine $(0.11 \mathrm{~g}$, $0.87 \mathrm{mmol})$ to give $10(0.09 \mathrm{~g}, 33 \%)$ as an off-white solid. LCMS (ESI): $\mathrm{m} / \mathrm{z}=384.15[\mathrm{M}+$ $\mathrm{H}]^{+}$. HRMS $\left(\mathrm{ESI}^{+}\right): \mathrm{m} / z$ calcd for $\mathrm{C}_{16} \mathrm{H}_{14} \mathrm{~F}_{4} \mathrm{~N}_{5} \mathrm{O}_{2}{ }^{+}(\mathrm{M}+\mathrm{H})^{+}, 384.1084$; found, 384.1068. ${ }^{1} \mathrm{H}$ NMR $(400 \mathrm{MHz}$, chloroform- $d$ ): $\delta 8.37(\mathrm{~d}, J=11.6 \mathrm{~Hz}, 1 \mathrm{H}), 8.26(\mathrm{~d}, J=8.0 \mathrm{~Hz}, 1 \mathrm{H})$, $8.18(\mathrm{~s}, 1 \mathrm{H}), 7.99(\mathrm{~s}, 1 \mathrm{H}), 7.94(\mathrm{~s}, 1 \mathrm{H}), 7.73-7.69(\mathrm{~m}, 1 \mathrm{H}), 7.59$ (br s, $1 \mathrm{H}), 4.32(\mathrm{t}, J=6.80$ $\mathrm{Hz}, 2 \mathrm{H}$ ), 3.53 (q, $J=6.40 \mathrm{~Hz}, 2 \mathrm{H}), 2.25$ (quin, $J=6.40 \mathrm{~Hz}, 2 \mathrm{H}) .{ }^{13} \mathrm{C} \mathrm{NMR}(100 \mathrm{MHz}$, $\mathrm{CDCl}_{3}$ ): $\delta 160.85,158.26,152.20,149.96,148.75(\mathrm{~d}, J=2.9 \mathrm{~Hz}), 143.30,132.32(\mathrm{~d}, J=9.3$ $\mathrm{Hz}), 130.68,127.29,123.54(\mathrm{~m}), 120.92,119.30(\mathrm{dd}, J=45.5$ and $12.6 \mathrm{~Hz}), 116.60(\mathrm{~d}, J=$ $24.5 \mathrm{~Hz}), 47.07,36.39$, 29.93. Purity: HPLC $t_{\mathrm{R}}=7.90 \mathrm{~min}$, HPLC purity $>99.4 \%$.

\section{N-(3-(1H-1,2,4-Triazol-1-yl)propyl)-5-(3,5-difluoro-4-methoxyphenyl)oxazole-4- carboxamide (11, OCM-11).-The title compound was synthesized from 5-(3,5-} difluoro-4-methoxyphenyl)-oxazole-4-carboxylic acid (0.2 g, $0.78 \mathrm{mmol})$ and 3-(1 H-1,2,4triazol-1-yl)propan-1-amine $(0.118 \mathrm{~g}, 0.93 \mathrm{mmol})$ to give $\mathbf{1 1}(0.09 \mathrm{~g}, 32 \%)$ as an off-white solid. LCMS (ESI): $\mathrm{m} / z=386.25[\mathrm{M}+\mathrm{Na}]^{+}$. HRMS (ESI $\left.{ }^{+}\right): \mathrm{m} / z$ calcd for $\mathrm{C}_{16} \mathrm{H}_{16} \mathrm{~F}_{2} \mathrm{~N}_{5} \mathrm{O}_{3}{ }^{+}$ $(\mathrm{M}+\mathrm{H})^{+}, 364.1221$; found, 364.1214. ${ }^{1} \mathrm{H}$ NMR $\left(400 \mathrm{MHz}\right.$, DMSO- $\left.d_{6}\right): \delta 8.65-8.69(\mathrm{~m}$, $1 \mathrm{H}), 8.64(\mathrm{~s}, 1 \mathrm{H}), 8.53(\mathrm{~s}, 1 \mathrm{H}), 8.19-8.10(\mathrm{~m}, 2 \mathrm{H}), 7.96(\mathrm{~s}, 1 \mathrm{H}), 4.21(\mathrm{t}, J=6.80 \mathrm{~Hz}, 2 \mathrm{H})$, 
4.01 (s, 3H), 3.27 (q, $J=6.40 \mathrm{~Hz}, 2 \mathrm{H}), 1.98-2.09$ (m, 2H). ${ }^{13} \mathrm{C}$ NMR (100 MHz, DMSO$\left.d_{6}\right): \delta 160.58,154.39(\mathrm{dd}, J=251$ and $6.8 \mathrm{~Hz}), 151.39,150.67,150.40,148.64,144.07$, 129.52, $121.59(\mathrm{~m}), 112.08(\mathrm{~m}), 61.76,46.52,36.03,29.38$. Purity: HPLC $t_{\mathrm{R}}=7.38 \mathrm{~min}$, HPLC purity $>96.6 \%$.

N-(3-(1H-1,2,4-Triazol-1-yl)propyl)-5-(3-fluoro-4-methoxyphenyl)oxazole-4carboxamide (12, OCM-12).-The title compound was synthesized from 5-(3-fluoro-4methoxyphenyl)-oxazole-4-carboxylic acid $(0.2 \mathrm{~g}, 0.84 \mathrm{mmol})$ and 3-( $1 \mathrm{H}-1,2,4-$ triazol-1yl)propan-1-amine $(0.127 \mathrm{~g}, 1.00 \mathrm{mmol})$ to give $\mathbf{1 2}(0.09 \mathrm{~g}, 31 \%)$ as an off-white solid. LCMS (ESI): $m / z=346.25[\mathrm{M}+\mathrm{H}]^{+}$. HRMS (ESI $\left.{ }^{+}\right): m / z$ calcd for $\mathrm{C}_{16} \mathrm{H}_{17} \mathrm{FN}_{5} \mathrm{O}_{3}{ }^{+}(\mathrm{M}+\mathrm{H})$ ${ }^{+}, 346.1315$; found, 346.1302. ${ }^{1} \mathrm{H}$ NMR $\left(400 \mathrm{MHz}\right.$, DMSO- $\left.d_{6}\right): \delta 8.59-8.51(\mathrm{~m}, 3 \mathrm{H}), 8.36-$ $8.33(\mathrm{~m}, 1 \mathrm{H}), 7.98(\mathrm{~s}, 1 \mathrm{H}), 7.96(\mathrm{~s}, 1 \mathrm{H}), 7.30(\mathrm{t}, J=8.80 \mathrm{~Hz}, 1 \mathrm{H}), 4.22(\mathrm{t}, J=6.80 \mathrm{~Hz}, 2 \mathrm{H})$, $3.91(\mathrm{~s}, 3 \mathrm{H}), 3.22-3.31(\mathrm{~m}, 2 \mathrm{H}), 2.04$ (quin, $J=6.80 \mathrm{~Hz}, 2 \mathrm{H}) .{ }^{13} \mathrm{C}$ NMR (100 MHz, DMSO$\left.d_{6}\right): \delta 160.87,151.39,150.78(\mathrm{~d}, J=241.3 \mathrm{~Hz}), 149.99,149.80,148.36(\mathrm{~d}, J=10.6 \mathrm{~Hz})$, $144.04,128.15,124.51,119.63(\mathrm{~d}, J=7.6 \mathrm{~Hz}), 115.53(\mathrm{~d}, J=21.5 \mathrm{~Hz}), 113.65,56.13$, 46.54, 35.96, 29.48. Purity: HPLC $t_{\mathrm{R}}=6.95 \mathrm{~min}$, HPLC purity $>96.7 \%$.

(Z)-5-(3-Fluoro-4-methoxyphenyl)-N-(3-phenylallyl)oxazole-4-carboxamide (13, OCM-13).-The title compound was synthesized from 5-(3-fluoro-4methoxyphenyl)oxazole-4-carboxylic acid $(0.2 \mathrm{~g}, 0.84 \mathrm{mmol})$ and $(Z)$-3-phenylprop-2-en-1amine $(0.134 \mathrm{~g}, 1.01 \mathrm{mmol})$ to get $\mathbf{1 3}(0.040 \mathrm{~g}, 13 \%)$ as an off-white solid. LCMS (ESI): $\mathrm{m} / \mathrm{z}=353.15[\mathrm{M}+\mathrm{H}]^{+}$. HRMS $\left(\mathrm{ESI}^{+}\right): \mathrm{m} / \mathrm{z}$ calcd for $\mathrm{C}_{20} \mathrm{H}_{18} \mathrm{FN}_{2} \mathrm{O}_{3}{ }^{+}(\mathrm{M}+\mathrm{H})^{+}, 353.1301$; found, 353.1294. ${ }^{1} \mathrm{H}$ NMR (400 MHz, $\left.\mathrm{CDCl}_{3}\right): \delta 8.21-8.18(\mathrm{~m}, 2 \mathrm{H}), 7.81(\mathrm{~s}, 1 \mathrm{H}), 7.40-7.28$ $(\mathrm{m}, 5 \mathrm{H}), 7.04(\mathrm{t}, J=8.8 \mathrm{~Hz}, 1 \mathrm{H}), 6.65(\mathrm{~d}, J=11.6 \mathrm{~Hz}, 1 \mathrm{H}), 5.83-5.77(\mathrm{~m}, 1 \mathrm{H}), 4.38(\mathrm{t}, J=6$ $\mathrm{Hz}, 2 \mathrm{H}), 3.96$ (s, 3H). ${ }^{13} \mathrm{C}$ NMR (100 MHz, $\left.\mathrm{CDCl}_{3}\right): \delta 160.95,151.84$ (d, $\left.J=243.8 \mathrm{~Hz}\right)$, $151.73,149.12(\mathrm{~d}, J=10.6 \mathrm{~Hz}), 147.48(\mathrm{~d}, J=4.6 \mathrm{~Hz}), 136.29,131.85,128.76,128.36$, 128.03, 127.75, 127.29, 124.91, 119.90 (d, $J=7.6 \mathrm{~Hz}), 116.04$ (d, $J=22 \mathrm{~Hz}), 112.83,56.22$, 37.68. Purity: HPLC $t_{\mathrm{R}}=8.99 \mathrm{~min}$, HPLC purity $>97.0 \%$.

\section{N-Cinnamyl-5-(3-fluoro-4-methoxyphenyl)oxazole-4-carboxamide (14, OCM-14).-The title compound was synthesized from 5-(3-fluoro-4-} methoxyphenyl)oxazole-4-carboxylic acid $(0.2 \mathrm{~g}, 0.84 \mathrm{mmol})$ and (E)-3-phenylprop-2-en-1amine $(0.134 \mathrm{~g}, 1.01 \mathrm{mmol})$ to get $\mathbf{1 4}(0.065 \mathrm{~g}, 21 \%)$ as an off-white solid. LCMS (ESI): $\mathrm{m} / \mathrm{z}=353.20[\mathrm{M}+\mathrm{H}]^{+}$. HRMS $\left(\mathrm{ESI}^{+}\right): \mathrm{m} / z$ calcd for $\mathrm{C}_{20} \mathrm{H}_{18} \mathrm{FN}_{2} \mathrm{O}_{3}{ }^{+}(\mathrm{M}+\mathrm{H})^{+}, 353.1301$; found, 353.1287. ${ }^{1} \mathrm{H}$ NMR (400 MHz, $\left.\mathrm{CDCl}_{3}\right): \delta 8.22-8.18(\mathrm{~m}, 2 \mathrm{H}), 7.82(\mathrm{~s}, 1 \mathrm{H}), 7.48-7.23$ $(\mathrm{m}, 6 \mathrm{H}), 7.05(\mathrm{t}, J=8.4 \mathrm{~Hz}, 1 \mathrm{H}), 6.63(\mathrm{~d}, J=16 \mathrm{~Hz}, 1 \mathrm{H}), 6.34-6.27(\mathrm{~m}, 1 \mathrm{H}), 4.25(\mathrm{t}, J=6$ $\mathrm{Hz}, 2 \mathrm{H}), 3.96$ (s, 3H). ${ }^{13} \mathrm{C} \mathrm{NMR}\left(100 \mathrm{MHz}, \mathrm{CDCl}_{3}\right): \delta 160.92,151.87(\mathrm{~d}, J=243.9 \mathrm{~Hz})$, 151.81, 149.15 (d, $J=10.5 \mathrm{~Hz}), 147.50$ (m), 136.55, 132.26, 128.56, 128.09, 127.70, 126.41, 125.32, 124.97 (d, $J=3.4 \mathrm{~Hz}), 119.93$ (d, $J=7.6 \mathrm{~Hz}), 116.08$ (d, $J=19.5 \mathrm{~Hz})$, 112.86, 56.24, 41.26. Purity: HPLC $t_{\mathrm{R}}=9.31 \mathrm{~min}$, HPLC purity $>99.4 \%$.

\section{5-(3-Fluoro-4-methoxyphenyl)-N-phenethyloxazole-4-carboxamide (15,} OCM-15).-The title compound was synthesized from 5-(3-fluoro-4methoxyphenyl)oxazole-4-carboxylic acid $(0.1 \mathrm{~g}, 0.42 \mathrm{mmol})$ and (2-phenylethan-1-amine $(0.061 \mathrm{~g}, 0.504 \mathrm{mmol})$ to get $\mathbf{1 5}(0.110 \mathrm{~g}, 76 \%)$ as an off-white solid. LCMS (ESI): $\mathrm{m} / \mathrm{z}=$ 
$341.10[\mathrm{M}+\mathrm{H}]^{+}$. HRMS $\left(\mathrm{ESI}^{+}\right): \mathrm{m} / z$ calcd for $\mathrm{C}_{19} \mathrm{H}_{18} \mathrm{FN}_{2} \mathrm{O}_{3}{ }^{+}(\mathrm{M}+\mathrm{H})^{+}, 341.1301$; found, 341.1295. ${ }^{1} \mathrm{H}$ NMR (400 MHz, DMSO- $\left.d_{6}\right): \delta 8.53(\mathrm{~s}, 1 \mathrm{H}), 8.44-8.41(\mathrm{~m}, 1 \mathrm{H}), 8.33(\mathrm{dd}, J=$ $1.6 \& 13.2 \mathrm{~Hz}, 1 \mathrm{H}), 7.95(\mathrm{~d}, J=8.8 \mathrm{~Hz}, 1 \mathrm{H}), 7.32-7.18(\mathrm{~m}, 6 \mathrm{H}), 3.91(\mathrm{~s}, 3 \mathrm{H}), 3.53-3.48(\mathrm{~m}$, $2 \mathrm{H}), 2.84(\mathrm{t}, J=7.2 \mathrm{~Hz}, 2 \mathrm{H}) .{ }^{13} \mathrm{C}$ NMR $\left(100 \mathrm{MHz}\right.$, DMSO- $\left.d_{6}\right): \delta 160.57,150.77$ (d, $J=$ $241.3 \mathrm{~Hz}), 149.88,149.76,148.33$ (d, $J=10.5 \mathrm{~Hz}), 139.33,128.59,128.32,128.18,126.08$, $124.44,119.60(\mathrm{~d}, J=8 \mathrm{~Hz}), 115.47(\mathrm{~d}, J=21.5 \mathrm{~Hz}), 113.64,56.09,35.08$. Purity: HPLC $t_{\mathrm{R}}$ $=9.26 \mathrm{~min}$, HPLC purity $>99.6 \%$.

5-(3-Fluoro-4-methoxyphenyl)-N-((1R,2R)-2-phenylcyclopropyl)-oxazole-4carboxamide and 5-(3-Fluoro-4-methoxyphenyl)-N-((1S,2S)-2-

phenylcyclopropyl)oxazole-4-carboxamide (16, OCM-16).-The title compound was synthesized from 5-(3-fluoro-4-methoxyphenyl)oxazole-4-carboxylic acid (0.2 g, 0.84 $\mathrm{mmol})$ and rac-cis-2-phenylcyclopropan-1-amine $(0.134 \mathrm{~g}, 1.01 \mathrm{mmol})$ to get $\mathbf{1 6}(0.110 \mathrm{~g}$, $36 \%$ ) as an off-white solid. LCMS (ESI): $\mathrm{m} / z=353.10[\mathrm{M}+\mathrm{H}]^{+}$. HRMS $\left(\mathrm{ESI}^{+}\right): \mathrm{m} / \mathrm{z}$ calcd for $\mathrm{C}_{20} \mathrm{H}_{18} \mathrm{FN}_{2} \mathrm{O}_{3}{ }^{+}(\mathrm{M}+\mathrm{H})^{+}, 353.1301$; found, 353.1290. ${ }^{1} \mathrm{H} \mathrm{NMR}\left(400 \mathrm{MHz}, \mathrm{CDCl}_{3}\right): \delta$ 8.16-8.13 (m, 2H), $7.65(\mathrm{~s}, 1 \mathrm{H}), 7.35-7.22(\mathrm{~m}, 5 \mathrm{H}), 7.04-6.97(\mathrm{~m}, 2 \mathrm{H}), 3.95(\mathrm{~s}, 3 \mathrm{H}), 3.34-$ $3.28(\mathrm{~m}, 1 \mathrm{H}), 2.47-2.41(\mathrm{~m}, 1 \mathrm{H}), 1.53-1.47(\mathrm{~m}, 1 \mathrm{H}), 1.20-1.16(\mathrm{~m}, 1 \mathrm{H}) .{ }^{13} \mathrm{C}$ NMR $(100$ $\left.\mathrm{MHz}, \mathrm{CDCl}_{3}\right): \delta 162.39,151.81(J=242 \mathrm{~Hz}), 151.38,149.05(\mathrm{~d}, J=11 \mathrm{~Hz}), 147.33(\mathrm{~d}, J=$ $5 \mathrm{~Hz}), 136.24,128.69,128.36,127.86,126.58,124.80,119.91$ (d, $J=8 \mathrm{~Hz}), 116.01$ (d, $J=$ $20 \mathrm{~Hz}), 112.79,56.19,28.57,21.85,12.71$. Purity: HPLC $t_{\mathrm{R}}=8.96 \mathrm{~min}$, HPLC purity > $99.8 \%$.

5-(3-Fluoro-4-methoxyphenyl)-N-((1S,2R)-2-phenylcyclopropyl)-oxazole-4carboxamide and 5-(3-Fluoro-4-methoxyphenyl)-N-((1R,2S)-2phenylcyclopropyl)oxazole-4-carboxamide (17, OCM-17).-The title compound was synthesized from 5-(3-fluoro-4-methoxyphenyl)oxazole-4-carboxylic acid (0.2 g, 0.84 $\mathrm{mmol})$ and rac-trans-2-phenylcyclopropan-1-amine $(0.134 \mathrm{~g}, 1 \mathrm{mmol})$ to get $17(0.100 \mathrm{~g}$, $33 \%$ ) as an off-white solid. LCMS (ESI): $\mathrm{m} / z=353.00[\mathrm{M}+\mathrm{H}]^{+}$. HRMS $\left(\mathrm{ESI}^{+}\right): \mathrm{m} / \mathrm{z}$ calcd for $\mathrm{C}_{20} \mathrm{H}_{18} \mathrm{FN}_{2} \mathrm{O}_{3}{ }^{+}(\mathrm{M}+\mathrm{H})^{+}, 353.1301$; found, 353.1297. ${ }^{1} \mathrm{H} \mathrm{NMR}\left(400 \mathrm{MHz}, \mathrm{CDCl}_{3}\right): \delta$ 8.25-8.17 (m, 2H), $7.81(\mathrm{~s}, 1 \mathrm{H}), 7.47(\mathrm{~s}, 1 \mathrm{H}), 7.33-7.29(\mathrm{~m}, 2 \mathrm{H}), 7.23-7.20(\mathrm{~m}, 3 \mathrm{H}), 7.05(\mathrm{t}$, $J=2.2 \mathrm{~Hz}, 1 \mathrm{H}), 3.96(\mathrm{~s}, 3 \mathrm{H}), 3.15-3.10(\mathrm{~m}, 1 \mathrm{H}), 2.24-2.19(\mathrm{~m}, 1 \mathrm{H}), 1.40-1.31(\mathrm{~m}, 2 \mathrm{H}) .{ }^{13} \mathrm{C}$ NMR (100 MHz, $\left.\mathrm{CDCl}_{3}\right): \delta 162.16,151.86(\mathrm{~d}, J=244.2 \mathrm{~Hz}), 151.77,149.18(\mathrm{~d}, J=10.6$ $\mathrm{Hz}), 147.43$ (d, $J=4.3 \mathrm{~Hz}), 140.41,128.41,127.91,126.42,126.16,125.01,119.83$ (d, $J=$ $8.1 \mathrm{~Hz}), 116.02(\mathrm{~d}, J=21.5 \mathrm{~Hz}), 112.86,56.23,31.88,24.70,16.38$. Purity: HPLC $t_{\mathrm{R}}=9.31$ min, HPLC purity $>99.9 \%$.

5-(3-Fluoro-4-methoxyphenyl)-N-(3-phenylpropyl)oxazole-4-carboxamide (18, OCM-18).-The title compound was synthesized from 5-(3-fluoro-4-

methoxyphenyl)oxazole-4-carboxylic acid $(0.2 \mathrm{~g}, 0.84 \mathrm{mmol})$ using commercial available amine 3-phenylpropan-1-amine (0.136 g, $1.01 \mathrm{mmol})$ to give $\mathbf{1 8}(0.09 \mathrm{~g}, 31 \%)$ as an offwhite solid. LCMS (ESI): $\mathrm{m} / z=355.10[\mathrm{M}+\mathrm{H}]^{+}$. HRMS $\left(\mathrm{ESI}^{+}\right): \mathrm{m} / z$ calcd for $\mathrm{C}_{20} \mathrm{H}_{20} \mathrm{FN}_{2} \mathrm{O}_{3}{ }^{+}(\mathrm{M}+\mathrm{H})^{+}, 355.1458$; found, 355.1453. ${ }^{1} \mathrm{H}$ NMR (400 MHz, DMSO- $\left.d_{6}\right): \delta$ $8.54(\mathrm{~s}, 1 \mathrm{H}), 8.47-8.44(\mathrm{~m}, 1 \mathrm{H}), 8.36-8.32(\mathrm{~m}, 1 \mathrm{H}), 7.96(\mathrm{~d}, J=8.8 \mathrm{~Hz}, 1 \mathrm{H}), 7.31-7.25(\mathrm{~m}$, $3 \mathrm{H}), 7.20-7.14(\mathrm{~m}, 3 \mathrm{H}), 3.89$ (s, 3H), 3.32-3.26 (m, 2H), $2.6(\mathrm{t}, J=7.20 \mathrm{~Hz}, 2 \mathrm{H}), 1.86-1.78$ (m, 2H). ${ }^{13} \mathrm{C} \mathrm{NMR}\left(100 \mathrm{MHz}, \mathrm{CDCl}_{3}\right): \delta 161.09,151.84(\mathrm{~d}, J=243.6 \mathrm{~Hz}), 151.58,149.08$ 
(d, $J=10.9 \mathrm{~Hz}), 147.45,141.35,128.44,128.37,128.21,125.96,124.93(\mathrm{~d}, J=3.4 \mathrm{~Hz})$, $119.98(\mathrm{~d}, J=7.5 \mathrm{~Hz}), 116.06$ (d, $J=21.5 \mathrm{~Hz}), 112.83,56.21,38.87,33.27,31.21$. Purity: HPLC $t_{\mathrm{R}}=8.83 \mathrm{~min}, \mathrm{HPLC}$ purity $>99.4 \%$.

5-(3-Fluoro-4-methoxyphenyl)-N-(3-(2-methyl-1H-imidazole-1yl)propyl)oxazole-4-carboxamide (19, PF-981).-PF-981 was graciously provided by Pfizer (HPLC purity > 95\%). Physical state: white solid. HRMS $\left(\mathrm{ESI}^{+}\right): \mathrm{m} / \mathrm{z}$ calcd for $\mathrm{C}_{18} \mathrm{H}_{20} \mathrm{FN}_{4} \mathrm{O}_{3}{ }^{+}(\mathrm{M}+\mathrm{H})^{+}$, 359.1519; found, 359.1514. ${ }^{1} \mathrm{H}$ NMR (400 MHz, $\left.\mathrm{CDCl}_{3}\right): \delta 8.14$ $(\mathrm{dt}, J=2.1,10.8 \mathrm{~Hz}, 2 \mathrm{H}), 7.82(\mathrm{~s}, 1 \mathrm{H}), 7.43(\mathrm{t}, J=5.8 \mathrm{~Hz}, 1 \mathrm{H}), 7.12(\mathrm{~d}, J=6.4 \mathrm{~Hz}, 2 \mathrm{H}), 7.05$ (t, $J=8.5 \mathrm{~Hz}, 1 \mathrm{H}), 4.05(\mathrm{t}, J=7.1 \mathrm{~Hz}, 2 \mathrm{H}), 3.95(\mathrm{~s}, 3 \mathrm{H}), 3.51(\mathrm{~m}, 2 \mathrm{H}), 2.62(\mathrm{~s}, 3 \mathrm{H}), 2.12(\mathrm{t}$, $J=6.8 \mathrm{~Hz}, 2 \mathrm{H}) .{ }^{13} \mathrm{C}$ NMR $\left(100 \mathrm{MHz}, \mathrm{CDCl}_{3}\right): \delta 161.8,153.1-150.6(\mathrm{~d}, J=245.5 \mathrm{~Hz})$, $152.1(\mathrm{~d}, J=2.7 \mathrm{~Hz}), 149.3(\mathrm{~d}, J=10.8 \mathrm{~Hz}), 147.7,144.1,127.5,124.9(\mathrm{~d}, J=3.6 \mathrm{~Hz})$, $122.3,119.9,119.5$ (d, $J=7.9 \mathrm{~Hz}), 116.0$ (d, $J=21.6 \mathrm{~Hz}), 112.9,56.3,44.5,36.1,30.7,11.7$.

5-(3-Fluoro-4-methoxyphenyl)-N-(3-(2-oxopyridin-1(2H)-yl)-propyl)oxazole-4carboxamide (20, PF-627).-PF-627 was graciously provided by Pfizer (HPLC purity > 95\%). Physical state: white solid. HRMS $\left(\mathrm{ESI}^{+}\right): \mathrm{m} / \mathrm{z}$ calcd for $\mathrm{C}_{19} \mathrm{H}_{19} \mathrm{FN}_{3} \mathrm{O}_{4}{ }^{+}(\mathrm{M}+\mathrm{H})^{+}$, 372.1360; found, 372.1367. ${ }^{1} \mathrm{H} \mathrm{NMR}\left(400 \mathrm{MHz}, \mathrm{CDCl}_{3}\right): \delta 8.21(\mathrm{dd}, J=12.8,2.1 \mathrm{~Hz}, 1 \mathrm{H})$, $8.12(\mathrm{~d}, J=8.8 \mathrm{~Hz}, 1 \mathrm{H}), 8.1(\mathrm{~s}, 1 \mathrm{H}), 7.84(\mathrm{~s}, 1 \mathrm{H}), 7.55(\mathrm{~d}, J=7.6 \mathrm{~Hz}, 1 \mathrm{H}), 7.49$ (d, $J=7.7$ $\mathrm{Hz}, 1 \mathrm{H}), 7.09-6.99(\mathrm{~m}, 2 \mathrm{H}), 6.45(\mathrm{t}, J=6.3 \mathrm{~Hz}, 1 \mathrm{H}), 4.17(\mathrm{t}, J=6.2 \mathrm{~Hz}, 2 \mathrm{H}), 3.94(\mathrm{~s}, 3 \mathrm{H})$, $3.47(\mathrm{~m}, 2 \mathrm{H}), 2.08(\mathrm{t}, J=6.1 \mathrm{~Hz}, 2 \mathrm{H}) .{ }^{13} \mathrm{C} \mathrm{NMR}\left(100 \mathrm{MHz}, \mathrm{CDCl}_{3}\right): \delta 163.1,161.5,153.1-$ $150.6(\mathrm{~d}, J=245.0 \mathrm{~Hz}), 151.5(\mathrm{~d}, J=2.4 \mathrm{~Hz}), 149.0(\mathrm{~d}, J=10.8 \mathrm{~Hz}), 147.8,140.4,137.7$, $128.3,124.8(\mathrm{~d}, J=3.6 \mathrm{~Hz}), 120.56,120.1(\mathrm{~d}, J=7.9 \mathrm{~Hz}), 116.2(\mathrm{~d}, J=21.7 \mathrm{~Hz}), 112.8$, $107.9,56.2,47.3,35.4,29.8$.

\section{5-(3-Fluoro-4-methoxyphenyl)-N-(3-(1-methyl-1H-pyrazol-4-yl)-} propyl)oxazole-4-carboxamide (21, PF-024).-PF-024 was graciously provided by Pfizer (HPLC purity > 95\%). Physical state: white solid. HRMS $\left(\mathrm{ESI}^{+}\right): \mathrm{m} / z$ calcd for $\mathrm{C}_{18} \mathrm{H}_{20} \mathrm{FN}_{4} \mathrm{O}_{3}{ }^{+}(\mathrm{M}+\mathrm{H})^{+}$, 359.1519; found, 359.1530. ${ }^{1} \mathrm{H}$ NMR (400 MHz, $\left.\mathrm{CDCl}_{3}\right): \delta$ $8.20-8.13(\mathrm{~m}, 2 \mathrm{H}), 7.79(\mathrm{~s}, 1 \mathrm{H}), 7.40(\mathrm{~s}, 1 \mathrm{H}), 7.31$ (br s, $1 \mathrm{H}), 7.02(\mathrm{t}, J=8.5 \mathrm{~Hz}, 1 \mathrm{H}) 3.94$ (s, 3H), $3.91(\mathrm{~s}, 3 \mathrm{H}), 3.46(\mathrm{~m}, 2 \mathrm{H}), 2.57(\mathrm{t}, J=7.5 \mathrm{~Hz}, 2 \mathrm{H}), 1.89(\mathrm{t}, J=7.3 \mathrm{~Hz}, 2 \mathrm{H}) .{ }^{13} \mathrm{C}$ NMR (100 MHz, $\left.\mathrm{CDCl}_{3}\right): \delta 161.1,153.1-150.6(\mathrm{~d}, J=245.1 \mathrm{~Hz}), 151.6(\mathrm{~d}, J=2.4 \mathrm{~Hz})$, $149.1(\mathrm{~d}, J=10.8 \mathrm{~Hz}), 147.5,137.5,129.0,128.2,124.9(\mathrm{~d}, J=3.6 \mathrm{~Hz}), 121.0,119.9(\mathrm{~d}, J=$ $7.9 \mathrm{~Hz}), 116.1$ (d, $J=21.7 \mathrm{~Hz}), 112.8(\mathrm{~d}, J=2.1 \mathrm{~Hz}), 56.2,38.7,38.6,30.8,21.5$.

\section{5-(3-Fluoro-4-methoxyphenyl)-N-(3-(2-methyl-1H-imidazol-4-} yl)propyl)oxazole-4-carboxamide (22, PF-760).-PF-760 was graciously provided by Pfizer (HPLC purity > 95\%). Physical state: White solid. HRMS $\left(\mathrm{ESI}^{+}\right): \mathrm{m} / \mathrm{z}$ calcd for $\mathrm{C}_{18} \mathrm{H}_{20} \mathrm{FN}_{4} \mathrm{O}_{3}{ }^{+}(\mathrm{M}+\mathrm{H})^{+}$, 359.1519; found, 359.1514. ${ }^{1} \mathrm{H} \mathrm{NMR}\left(400 \mathrm{MHz}, \mathrm{CDCl}_{3}\right): \delta 8.20$ $(\mathrm{dd}, J=12.9,2.0 \mathrm{~Hz}, 1 \mathrm{H}), 8.01(\mathrm{~d}, J=8.6 \mathrm{~Hz}, 1 \mathrm{H}), 7.84(\mathrm{~s}, 1 \mathrm{H}), 7.62(\mathrm{t}, J=6.3 \mathrm{~Hz}, 1 \mathrm{H})$, $7.02(\mathrm{t}, J=8.6 \mathrm{~Hz}, 1 \mathrm{H}), 6.87(\mathrm{~s}, 1 \mathrm{H}), 3.94(\mathrm{~s}, 3 \mathrm{H}), 3.50(\mathrm{~m}, 2 \mathrm{H}), 2.71(\mathrm{~s}, 3 \mathrm{H}), 1.98(\mathrm{t}, J=6.5$ $\mathrm{Hz}, 2 \mathrm{H}), 1.25$ (s, 2H). ${ }^{13} \mathrm{C} \mathrm{NMR}\left(100 \mathrm{MHz}, \mathrm{CDCl}_{3}\right): \delta 162.3,153.0-150.5$ (d, $J=245.1$ $\mathrm{Hz}), 152.0(\mathrm{~d}, J=2.4 \mathrm{~Hz}), 149.3(\mathrm{~d}, J=10.7 \mathrm{~Hz}), 147.8,143.3,132.2,127.5,124.8$ (d, $J=$ $3.4 \mathrm{~Hz}), 119.6(\mathrm{~d}, J=8.0 \mathrm{~Hz}), 116.3(\mathrm{~d}, J=21.8 \mathrm{~Hz}), 155.0,112.9,56.3,38.0,29.0,21.5$, 11.2 . 
5-(3-Fluoro-4-(trifluoromethyl)phenyl)-N-(3-(pyrazin-2-yl)-propyl)oxazole-4carboxamide (23, OCM-23).-The title compound was synthesized from 5-(3-fluoro-4(trifluoromethyl)phenyl)oxazole-4-carboxylic acid $(0.2 \mathrm{~g}, 0.72 \mathrm{mmol})$ and 3-(pyrazin-2yl)propan-1-amine $(0.12 \mathrm{~g}, 0.87 \mathrm{mmol})$ to give $23(0.085 \mathrm{~g}, 30 \%)$ as an off-white solid. LCMS (ESI): $m / z=395.15[\mathrm{M}+\mathrm{H}]^{+}$. HRMS $\left(\mathrm{ESI}^{+}\right): \mathrm{m} / z$ calcd for $\mathrm{C}_{18} \mathrm{H}_{15} \mathrm{~F}_{4} \mathrm{~N}_{4} \mathrm{O}_{4}{ }^{+}(\mathrm{M}+$ $\mathrm{H})^{+}, 395.1131$; found, 395.1138. ${ }^{1} \mathrm{H}$ NMR (400 MHz, chloroform- $d$ ): $\delta 8.52-8.50(\mathrm{~m}, 2 \mathrm{H})$, $8.42(\mathrm{~s}, 1 \mathrm{H}), 8.38(\mathrm{~d}, J=12.00 \mathrm{~Hz}, 1 \mathrm{H}), 8.25(\mathrm{~d}, J=8.00 \mathrm{~Hz}, 1 \mathrm{H}), 7.91(\mathrm{~s}, 1 \mathrm{H}), 7.68(\mathrm{t}, J=$ $7.60 \mathrm{~Hz}, 1 \mathrm{H}), 7.59$ (br s, 1H), 3.55 (q, $J=6.80 \mathrm{~Hz}, 2 \mathrm{H}), 2.95$ (t, $J=7.60 \mathrm{~Hz}, 2 \mathrm{H}), 2.13$ (quin, $J=7.20 \mathrm{~Hz}, 2 \mathrm{H}) .{ }^{13} \mathrm{C} \mathrm{NMR}\left(100 \mathrm{MHz}, \mathrm{CDCl}_{3}\right): \delta 160.52,159.52(\mathrm{~d}, J=253 \mathrm{~Hz}$ ), 156.61, 149.80, 148.65, 144.61, 144.04, 142.43, 132.46 (d, $J=9.3 \mathrm{~Hz}), 130.68,127.19$, $123.54(\mathrm{~m}), 120.92,119.30$ (dd, $J=45.5$ and $12.6 \mathrm{~Hz}), 116.60(\mathrm{~d}, J=24.4 \mathrm{~Hz}), 38.83$, 32.63 , 28.67. Purity: HPLC $t_{\mathrm{R}}=8.52 \mathrm{~min}$, HPLC purity $>95.3 \%$.

N-(3-(Pyrazin-2-yl)propyl)-5-(3,4,5-trifluorophenyl)oxazole-4-carboxamide (24, OCM-24).-The title compound was synthesized from 5-(3,4,5-trifluorophenyl)oxazole-4carboxylic acid $(0.1 \mathrm{~g}, 0.41 \mathrm{mmol})$ and 3-(pyrazin-2-yl)propan-1-amine $(0.068 \mathrm{~g}, 0.49$ $\mathrm{mmol})$ to give $24(0.042 \mathrm{~g}, 29 \%)$ as an off-white solid. LCMS (ESI): $\mathrm{m} / \mathrm{z}=363.15[\mathrm{M}+\mathrm{H}]$ + . HRMS $\left(\mathrm{ESI}^{+}\right): \mathrm{m} / \mathrm{z}$ calcd for $\mathrm{C}_{17} \mathrm{H}_{14} \mathrm{~F}_{3} \mathrm{~N}_{4} \mathrm{O}_{2}{ }^{+}(\mathrm{M}+\mathrm{H})^{+}, 369.1069$; found, 369.1075. ${ }^{1} \mathrm{H}$ NMR (400 MHz, DMSO- $\left.d_{6}\right): \delta 8.52-8.46(\mathrm{~m}, 2 \mathrm{H}), 8.41$ (br s, $\left.1 \mathrm{H}\right), 8.20-8.15(\mathrm{~m}, 2 \mathrm{H}), 7.84$ (s, 1H), 7.55 (s, 1H), 3.53 (q, $J=6.8 \mathrm{~Hz}, 2 \mathrm{H}), 2.92(\mathrm{t}, J=7.6 \mathrm{~Hz}, 2 \mathrm{H}), 2.14-2.07$ (m, 2H). ${ }^{13} \mathrm{C}$ NMR $\left(100 \mathrm{MHz}, \mathrm{CDCl}_{3}\right): \delta 160.66,156.61,152.25(\mathrm{~m}), 149.74(\mathrm{~m}), 148.15(\mathrm{~m})$, 144.62, 144.04, 142.44, 134.64 (dt, $J=252.5$ and $16.0 \mathrm{~Hz}), 129.94,122.78(\mathrm{~m}), 112.71(\mathrm{~m})$, 38.81, 32.62, 28.70. Purity: HPLC $t_{\mathrm{R}}=8.20 \mathrm{~min}$, $\mathrm{HPLC}$ purity $>99.7 \%$.

\section{5-(3,4-Difluorophenyl)-N-(3-(pyrazin-2-yl)propyl)oxazole-4-carboxamide (25,} OCM-25).-The title compound was synthesized from 5-(3,4-difluorophenyl)oxazole-4carboxylic acid $(0.15 \mathrm{~g}, 0.66 \mathrm{mmol})$ and 3-(pyrazin-2-yl)propan-1-amine $(0.11 \mathrm{~g}, 0.80$ mmol) to give 25 was obtained ( $0.1 \mathrm{~g}, 43 \%$ ), as an off-white solid. LCMS (ESI): $\mathrm{m} / z=$ $345.30[\mathrm{M}+\mathrm{H}]^{+}$. HRMS $\left(\mathrm{ESI}^{+}\right): \mathrm{m} / z$ calcd for $\mathrm{C}_{17} \mathrm{H}_{15} \mathrm{~F}_{2} \mathrm{~N}_{4} \mathrm{O}_{2}{ }^{+}(\mathrm{M}+\mathrm{H})^{+}, 345.1163$; found, 345.1161. ${ }^{1} \mathrm{H}$ NMR (400 MHz, DMSO- $\left.d_{6}\right): \delta 8.52-8.50(\mathrm{~m}, 2 \mathrm{H}), 8.42(\mathrm{~d}, J=2.4 \mathrm{~Hz}, 1 \mathrm{H})$, 8.39-8.34 (m, 1H), 8.16-8.13 (m, 1H), $7.85(\mathrm{~s}, 1 \mathrm{H}), 7.60-7.50(\mathrm{~m}, 1 \mathrm{H}), 7.30-7.20(\mathrm{~m}, 1 \mathrm{H})$, $3.58-3.53(\mathrm{~m}, 2 \mathrm{H}), 2.95(\mathrm{t}, J=7.60 \mathrm{~Hz}, 2 \mathrm{H}), 2.13$ (quin, $J=7.20 \mathrm{~Hz}, 2 \mathrm{H}) .{ }^{13} \mathrm{C}$ NMR $(100$ MHz, DMSO- $\left.d_{6}\right): \delta 160.46,156.78,150.42,150.20(\mathrm{~m}), 148.96(\mathrm{~m}), 147.83,144.46,143.98$ (d, $J=13.1 \mathrm{~Hz}), 142.28,129.47,124.88(\mathrm{~m}), 124.3$ (dd, $J=11$ and $3.48 \mathrm{~Hz}), 117.83$ (d, $J=$ 17.7 Hz), 117.24 (d, $J=20.3 \mathrm{~Hz}), 38.32,32.05,28.46$. Purity: HPLC $t_{\mathrm{R}}=7.79 \mathrm{~min}, \mathrm{HPLC}$ purity $>99.0 \%$.

\section{N-(3-(Pyrazin-2-yl)propyl)-5-(3,4,5-trifluorophenyl)oxazole-4-carboxamide (26,} OCM-26).-The title compound was synthesized from 5-(3,5-difluoro-4methoxyphenyl)oxazole-4-carboxylic acid $(0.2 \mathrm{~g}, 0.78 \mathrm{mmol})$ and 3-(pyrazin-2-yl)propan-1amine $(0.128 \mathrm{~g}, 0.93 \mathrm{mmol})$ to give $\mathbf{2 6}(0.070 \mathrm{~g}, 24 \%)$ as an off-white solid. LCMS (ESI): $\mathrm{m} / \mathrm{z}=375.15[\mathrm{M}+\mathrm{H}]^{+}$. HRMS $\left(\mathrm{ESI}^{+}\right): \mathrm{m} / \mathrm{z}$ calcd for $\mathrm{C}_{18} \mathrm{H}_{17} \mathrm{~F}_{2} \mathrm{~N}_{4} \mathrm{O}_{3}{ }^{+}(\mathrm{M}+\mathrm{H})^{+}, 375.1269$; found, 375.1267. ${ }^{1} \mathrm{H}$ NMR (400 MHz, DMSO- $\left.d_{6}\right): \delta 8.60(\mathrm{~s}, 1 \mathrm{H}), 8.58-8.56(\mathrm{~m}, 1 \mathrm{H}), 8.54$ $8.50(\mathrm{~m}, 1 \mathrm{H}), 8.44-8.42(\mathrm{~m}, 1 \mathrm{H}), 8.15-8.08(\mathrm{~m}, 2 \mathrm{H}), 4.00(\mathrm{~s}, 3 \mathrm{H}), 3.36-3.31(\mathrm{~m}, 2 \mathrm{H}), 2.80$ (t, $J=7.60 \mathrm{~Hz}, 2 \mathrm{H}), 1.98-1,91(\mathrm{~m}, 2 \mathrm{H}) .{ }^{13} \mathrm{C}$ NMR (100 MHz, DMSO- $\left.d_{6}\right): \delta 160.41,156.77$, 
$154.38(\mathrm{dd}, J=244.9$ and $6.4 \mathrm{~Hz}), 150.34,148.51,144.45,143.95,142.27,129.62,121.66$ (m), $112.02(\mathrm{~m}), 61.78,38.33,32.04,28.43$. Purity: HPLC $t_{\mathrm{R}}=7.98 \mathrm{~min}$, HPLC purity > 95.3\%.

5-(3-Fluoro-4-hydroxyphenyl)-N-(3-(pyrazin-2-yl)propyl)-oxazole-4carboxamide (27, PF-796).-PF-796 was graciously provided by Pfizer (HPLC purity > 95\%). Physical state: white solid. HRMS (ESI $\left.{ }^{+}\right): m / z$ calcd for $\mathrm{C}_{18} \mathrm{H}_{20} \mathrm{FN}_{4} \mathrm{O}_{3}{ }^{+}(\mathrm{M}+\mathrm{H})^{+}$, 343.1206; found, 343.1206. ${ }^{1} \mathrm{H}$ NMR $\left(400 \mathrm{MHz}, \mathrm{CD}_{3} \mathrm{OD}\right): \delta 8.74-8.66(\mathrm{~m}, 1 \mathrm{H}), 8.55(\mathrm{~d}, J$ $=2.7 \mathrm{~Hz}, 1 \mathrm{H}), 8.23-8.07(\mathrm{~m}, 2 \mathrm{H}), 7.83(\mathrm{~d}, J=6.6 \mathrm{~Hz}, 1 \mathrm{H}), 6.99(\mathrm{t}, J=8.8 \mathrm{~Hz}, 1 \mathrm{H}), 3.47(\mathrm{t}$, $J=6.8 \mathrm{~Hz}, 2 \mathrm{H}), 3.01(\mathrm{t}, J=7.5 \mathrm{~Hz}, 2 \mathrm{H}), 2.11(\mathrm{t}, J=7.6 \mathrm{~Hz}, 2 \mathrm{H}) .{ }^{13} \mathrm{C} \mathrm{NMR}(100 \mathrm{MHz}$, $\left.\mathrm{CD}_{3} \mathrm{OD}\right): \delta 162.2,157.6,151.8-149.9(\mathrm{~d}, J=239.8 \mathrm{~Hz}), 151.6(\mathrm{~d}, J=2.8 \mathrm{~Hz}), 148.7,146.9$, 143.5, 143.3, 140.7, 127.2, 124.4, 118.7 (d, $J=7.7 \mathrm{~Hz}), 117.2,115.9$ (d, $J=22.1 \mathrm{~Hz}), 38.2$, $31.8,28.4$.

5-(3-Fluoro-4-methoxyphenyl)-N-(3-(pyrazin-2-yl)propyl)-oxazole-4carboxamide (28, OCM-28).-The title compound was synthesized from 5-(3-fluoro-4methoxyphenyl)oxazole-4-carboxylic acid $(0.2 \mathrm{~g}, 0.84 \mathrm{mmol})$ and 3-(pyrazin-2-yl)propan-1amine $(0.138 \mathrm{~g}, 1.00 \mathrm{mmol})$ to give $\mathbf{2 8}(0.060 \mathrm{~g}, 20 \%)$ as an off-white solid. LCMS (ESI): $\mathrm{m} / \mathrm{z}=357.10[\mathrm{M}+\mathrm{H}]^{+}$. HRMS $\left(\mathrm{ESI}^{+}\right): \mathrm{m} / \mathrm{z}$ calcd for $\mathrm{C}_{18} \mathrm{H}_{18} \mathrm{FN}_{4} \mathrm{O}_{3}{ }^{+}(\mathrm{M}+\mathrm{H})^{+}, 357.1363$; found, 357.1369. ${ }^{1} \mathrm{H}$ NMR (400 MHz, DMSO- $d_{6}$ ): $\delta 8.58(\mathrm{~s}, 1 \mathrm{H}), 8.54(\mathrm{~s}, 2 \mathrm{H}), 8.50(\mathrm{t}, J=$ $6.00 \mathrm{~Hz}, 1 \mathrm{H}), 8.45(\mathrm{~d}, J=2.40 \mathrm{~Hz}, 1 \mathrm{H}), 8.35(\mathrm{dd}, J=2.00,13.60 \mathrm{~Hz}, 1 \mathrm{H}), 7.96(\mathrm{~d}, J=8.80$ $\mathrm{Hz}, 1 \mathrm{H}), 7.29$ (t, $J=8.80 \mathrm{~Hz}, 1 \mathrm{H}), 3.91(\mathrm{~s}, 3 \mathrm{H}), 3.35-3.30(\mathrm{~m}, 2 \mathrm{H}), 2.81(\mathrm{t}, J=7.6 \mathrm{~Hz}, 2 \mathrm{H})$, 1.95 (quin, $J=7.20 \mathrm{~Hz}, 2 \mathrm{H}$ ). ${ }^{13} \mathrm{C}$ NMR (100 MHz, DMSO- $\left.d_{6}\right): \delta 160.69,156.86,150.77(\mathrm{~d}$, $J=241.3 \mathrm{~Hz}), 149.84,149.75,148.31$ (d, $J=10.9 \mathrm{~Hz}), 144.45,143.96,142.27,128.25$, $124.45,119.66$ (d, $J=8 \mathrm{~Hz}), 115.48$ (d, $J=21.5 \mathrm{~Hz}), 113.65,56.18,38.26,32.06,28.51$. Purity: HPLC $t_{\mathrm{R}}=7.53 \mathrm{~min}$, HPLC purity $>99.7 \%$.

5-(3-Fluoro-4-(trifluoromethoxy)phenyl)-N-(3-(pyrazin-2-yl)-propyl)oxazole-4carboxamide (29, OCM-29).-The title compound was synthesized from 5-(3-fluoro-4(trifluoromethoxy)phenyl)-oxazole-4-carboxylic acid (0.15 g, $0.51 \mathrm{mmol})$ and 3-(pyrazin-2yl)propan-1-amine $(0.08 \mathrm{~g}, 0.61 \mathrm{mmol})$ to give $29(0.11 \mathrm{~g}, 52 \%)$ as an off-white solid. LCMS (ESI): $m / z=411.20[\mathrm{M}+\mathrm{H}]^{+}$. HRMS $\left(\mathrm{ESI}^{+}\right): \mathrm{m} / z$ calcd for $\mathrm{C}_{18} \mathrm{H}_{15} \mathrm{~F}_{4} \mathrm{~N}_{4} \mathrm{O}_{3}{ }^{+}(\mathrm{M}+$ $\mathrm{H})^{+}, 411.1080$; found, 411.1077. ${ }^{1} \mathrm{H}$ NMR $(400 \mathrm{MHz}$, chloroform- $d$ ): $\delta 8.53-8.51(\mathrm{~m}, 2 \mathrm{H})$, $8.43(\mathrm{~d}, J=2.40 \mathrm{~Hz}, 1 \mathrm{H}), 8.40(\mathrm{dd}, J=1.60,11.60 \mathrm{~Hz}, 1 \mathrm{H}), 8.19(\mathrm{~d}, J=8.80 \mathrm{~Hz}, 1 \mathrm{H}), 7.89$ (s, 1H), 7.58 (br s, 1H), 7.40 (t, $J=8.00 \mathrm{~Hz}, 1 \mathrm{H}), 3.56(\mathrm{q}, J=7.2 \mathrm{~Hz}, 2 \mathrm{H}), 2.96$ (t, $J=7.60$ $\mathrm{Hz}, 2 \mathrm{H}), 2.17-2.10(\mathrm{~m}, 2 \mathrm{H}) .{ }^{13} \mathrm{C}$ NMR (100 MHz, DMSO- $\left.d_{6}\right): \delta 160.35,156.78(\mathrm{~d}, J=$ $246.8 \mathrm{~Hz}), 150.88,148.40,144.47,143.98,142.30,135.76$ (d, $J=14.4 \mathrm{~Hz}), 130.33,128.01$, 127.92, $124.81(\mathrm{~m}), 124.09,120.15(\mathrm{~d}, J=210 \mathrm{~Hz}), 117.05(\mathrm{~d}, J=22.3 \mathrm{~Hz}), 38.35,32.06$, 28.44. Purity: HPLC $t_{\mathrm{R}}=8.66 \mathrm{~min}, \mathrm{HPLC}$ purity $>98.9 \%$.

5-(4-Cyano-3-fluorophenyl)-N-(3-(pyrazin-2-yl)propyl)oxazole-4-carboxamide (30, OCM-30).-The title compound was synthesized from 5-(4-cyano-3fluorophenyl)oxazole-4-carboxylic acid $(0.15 \mathrm{~g}, 0.65 \mathrm{mmol})$ and 3-(pyrazin-2-yl)propan-1amine $(0.11 \mathrm{~g}, 0.78 \mathrm{mmol})$ to give $\mathbf{3 0}(0.07 \mathrm{~g}, 31 \%)$ as an off-white solid. LCMS (ESI): $\mathrm{m} / \mathrm{z}$ $=352.10[\mathrm{M}+\mathrm{H}]^{+}$. HRMS $\left(\mathrm{ESI}^{+}\right): \mathrm{m} / \mathrm{z}$ calcd for $\mathrm{C}_{18} \mathrm{H}_{15} \mathrm{FN}_{5} \mathrm{O}_{2}{ }^{+}(\mathrm{M}+\mathrm{H})^{+}, 352.1210$; 
found, 352.1206. ${ }^{1} \mathrm{H}$ NMR (400 MHz, chloroform- $d$ ): $\delta 8.53-8.43$ (m, 4H), 8.32 (dd, $J=$ $1.47,8.31 \mathrm{~Hz}, 1 \mathrm{H}), 7.93(\mathrm{~s}, 1 \mathrm{H}), 7.70(\mathrm{dd}, J=6.85,8.31 \mathrm{~Hz}, 1 \mathrm{H}), 7.63(\mathrm{br} \mathrm{s}, 1 \mathrm{H}), 3.55$ (q, $J$ $=6.36 \mathrm{~Hz}, 2 \mathrm{H}), 2.94(\mathrm{t}, J=7.58 \mathrm{~Hz}, 2 \mathrm{H}), 2.17-2.10(\mathrm{~m}, 2 \mathrm{H}) .{ }^{13} \mathrm{C} \mathrm{NMR}(100 \mathrm{MHz}$, chloroform- $d$ ): $\delta 162.9$ (d, $J=257 \mathrm{~Hz}), 160.4,156.5,149.0,148.9(\mathrm{~d}, J=3 \mathrm{~Hz}), 144.6$, 144.1, 142.5, 133.4, 133.26, 131.7, 124.0 (d, $J=4 \mathrm{~Hz}), 116.0$ (d, $J=24 \mathrm{~Hz}), 113.7,102.1$ (d, $J=15.6 \mathrm{~Hz}), 38.9,32.6,28.6$. Purity: HPLC $t_{\mathrm{R}}=7.53 \mathrm{~min}$, HPLC purity $>99.4 \%$.

\section{5-(3-Cyano-4-fluorophenyl)-N-(3-(pyrazin-2-yl)propyl)oxazole-4-carboxamide (31, OCM-31).-The title compound was synthesized from 5-(3-cyano-4-}

fluorophenyl)oxazole-4-carboxylic acid (0.15 g, $0.65 \mathrm{mmol})$ and 3-(pyrazin-2-yl)propan-1amine $(0.11 \mathrm{~g}, 0.78 \mathrm{mmol})$ to give $31(0.045 \mathrm{~g}, 20 \%)$ as an off-white solid. LCMS (ESI) = $352.05[\mathrm{M}+\mathrm{H}]^{+}$. HRMS $\left(\mathrm{ESI}^{+}\right): \mathrm{m} / z$ calcd for $\mathrm{C}_{18} \mathrm{H}_{15} \mathrm{FN}_{5} \mathrm{O}_{2}{ }^{+}(\mathrm{M}+\mathrm{H})^{+}, 352.1210$; found, 352.1216. ${ }^{1} \mathrm{H}$ NMR (400 MHz, chloroform- $d$ ): $\delta 8.77-8.73(\mathrm{~m}, 1 \mathrm{H}), 8.65(\mathrm{dd}, J=2.20,6.11$ $\mathrm{Hz}, 1 \mathrm{H}), 8.53-8.48(\mathrm{~m}, 2 \mathrm{H}), 8.42(\mathrm{~d}, J=2.45 \mathrm{~Hz}, 1 \mathrm{H}), 7.89(\mathrm{~s}, 1 \mathrm{H}), 7.60(\mathrm{br} \mathrm{s}, 1 \mathrm{H}), 7.32(\mathrm{t}$, $J=8.56 \mathrm{~Hz}, 1 \mathrm{H}), 3.55(\mathrm{q}, J=6.85 \mathrm{~Hz}, 2 \mathrm{H}), 2.94(\mathrm{t}, J=7.58 \mathrm{~Hz}, 2 \mathrm{H}), 2.17-2.09(\mathrm{~m}, 2 \mathrm{H})$.

${ }^{13} \mathrm{C}$ NMR (100 MHz, chloroform- $d$ ): $\delta 163.4(\mathrm{~d}, J=262.4 \mathrm{~Hz}), 160.6,156.6,149.5,148.4$ (d, $J=4 \mathrm{~Hz}), 144.6,144.0,142.5,135.1$ (d, $J=9 \mathrm{~Hz}), 133.4,129.9,124.4$ (d, $J=3 \mathrm{~Hz})$, $116.7(\mathrm{~d}, J=20 \mathrm{~Hz}), 113.4,102.0(\mathrm{~d}, J=16 \mathrm{~Hz}), 38.8,32.6,28.7$. Purity: HPLC $t_{\mathrm{R}}=7.45$ min, HPLC Purity $>99.7 \%$.

\section{5-(3-lodo-4-methoxyphenyl)-N-(3-(pyrazin-2-yl)propyl)oxazole-4-carboxamide (32, OCM-32).-The title compound was synthesized from 5-(3-iodo-4-} methoxyphenyl)oxazole-4-carboxylic acid $(0.35 \mathrm{~g}, 1.00 \mathrm{mmol})$ and 3-(pyrazin-2yl)propan-1-amine $(0.16 \mathrm{~g}, 1.20 \mathrm{mmol})$ to give $32(0.18 \mathrm{~g}, 38 \%)$ as a pale yellow solid. LCMS $($ ESI $)=465.05[\mathrm{M}+\mathrm{H}]^{+}$. HRMS $\left(\mathrm{ESI}^{+}\right): \mathrm{m} / z$ calcd for $\mathrm{C}_{18} \mathrm{H}_{18} \mathrm{IN}_{4} \mathrm{O}_{3}{ }^{+}(\mathrm{M}+\mathrm{H})^{+}$, 465.0424; found, 465.0410. ${ }^{1} \mathrm{H}$ NMR (400 MHz, chloroform- $d$ ): $\delta 8.64(\mathrm{~d}, J=1.96 \mathrm{~Hz}, 1 \mathrm{H})$, $8.53-8.48(\mathrm{~m}, 3 \mathrm{H}), 8.41(\mathrm{~d}, J=2.45 \mathrm{~Hz}, 1 \mathrm{H}), 7.80(\mathrm{~s}, 1 \mathrm{H}), 7.48$ (br s, $1 \mathrm{H}), 6.89$ (d, $J=8.80$ $\mathrm{Hz}, 1 \mathrm{H}), 3.94$ (s, 3H), 3.54 (q, $J=6.85 \mathrm{~Hz}, 2 \mathrm{H}), 2.93$ (t, $J=7.58 \mathrm{~Hz}, 2 \mathrm{H}), 2.15-2.06$ (m, $2 \mathrm{H}) .{ }^{13} \mathrm{C}$ NMR (100 MHz, chloroform- $d$ ): $\delta 160.5,158.6,156.1,150.6,146.9,143.9,143.4$, 141.7, 138.2, 129.7, 127.3, 120.8, 109.6, 84.8, 55.8, 38.0, 32.0, 28.2. Purity: HPLC $t_{\mathrm{R}}=8.24$ min, HPLC Purity $>98.8 \%$.

\section{5-(3-Fluoro-4-(trifluoromethyl)phenyl)-N-(3-(pyridin-2-yl)-propyl)oxazole-4-} carboxamide (33, OCM-33).-The title compound was synthesized from 5-(3-fluoro-4(trifluoromethyl)phenyl)oxazole-4-carboxylic acid $(0.2 \mathrm{~g}, 0.72 \mathrm{mmol})$ and 3-(pyridin-2yl)propan-1-amine $(0.12 \mathrm{~g}, 0.87 \mathrm{mmol})$ to give $33(0.1 \mathrm{~g}, 35 \%)$ as an off-white solid. LCMS (ESI): $m / z=394.15[\mathrm{M}+\mathrm{H}]^{+}$. HRMS $\left(\mathrm{ESI}^{+}\right): \mathrm{m} / z$ calcd for $\mathrm{C}_{19} \mathrm{H}_{16} \mathrm{~F}_{4} \mathrm{~N}_{3} \mathrm{O}_{2}{ }^{+}(\mathrm{M}+\mathrm{H})^{+}$, 394.1179; found, 394.1166. ${ }^{1} \mathrm{H}$ NMR (400 MHz, chloroform- $d$ ): $\delta 8.56$ (d, $\left.J=4.40 \mathrm{~Hz}, 1 \mathrm{H}\right)$, $8.39(\mathrm{~d}, J=12.00 \mathrm{~Hz}, 1 \mathrm{H}), 8.26(\mathrm{~d}, J=8.00 \mathrm{~Hz}, 1 \mathrm{H}), 7.92(\mathrm{~s}, 1 \mathrm{H}), 7.76(\mathrm{br} \mathrm{s}, 1 \mathrm{H}), 7.71$ (t, $J$ $=14.2 \mathrm{~Hz}, 1 \mathrm{H}), 7.60(\mathrm{dt}, J=1.60,7.60 \mathrm{~Hz}, 1 \mathrm{H}), 7.19(\mathrm{~d}, J=8.00 \mathrm{~Hz}, 1 \mathrm{H}), 7.15-7.12(\mathrm{~m}$, $1 \mathrm{H}$ ), $3.53\left(\mathrm{q}, J=6.50 \mathrm{~Hz}, 2 \mathrm{H}\right.$ ), $2.92\left(\mathrm{t}, J=7.40 \mathrm{~Hz}, 2 \mathrm{H}\right.$ ), 2.11 (quin, $J=7.10 \mathrm{~Hz}, 2 \mathrm{H}$ ). ${ }^{13} \mathrm{C}$ NMR not assigned. Purity: HPLC $t_{\mathrm{R}}=6.27 \mathrm{~min}$, HPLC purity $>96.5 \%$.

N-(3-(Pyridin-2-yl)propyl)-5-(3,4,5-trifluorophenyl)oxazole-4-carboxamide (34, OCM-34).-The title compound was synthesized from 5-(3,4,5-trifluorophenyl)oxazole-4- 
carboxylic acid (0.2 g, $0.82 \mathrm{mmol})$ and 3-(pyridin-2-yl)propan-1-amine $(0.135 \mathrm{~g}, 1.00$ $\mathrm{mmol})$ to give $34(0.030 \mathrm{~g}, 10 \%)$ as an off-white solid. LCMS (ESI): $\mathrm{m} / \mathrm{z}=362.10[\mathrm{M}+\mathrm{H}]$ ${ }^{+}$. HRMS $\left(\mathrm{ESI}^{+}\right): \mathrm{m} / z$ calcd for $\mathrm{C}_{18} \mathrm{H}_{15} \mathrm{~F}_{3} \mathrm{~N}_{3} \mathrm{O}_{2}{ }^{+}(\mathrm{M}+\mathrm{H})^{+}, 362.1116$; found, 362.1109. ${ }^{1} \mathrm{H}$ NMR (400 MHz, chloroform- $d$ ): $\delta 8.56(\mathrm{~d}, J=4.4 \mathrm{~Hz}, 1 \mathrm{H}), 8.30-8.15(\mathrm{~m}, 2 \mathrm{H}), 7.86(\mathrm{~s}, 1 \mathrm{H})$, 7.73 (br s, 1H), 7.64-7.60 (m, 1H), 7.21-7.19 (m, 1H), 7.15-7.12 (m, 1H), 3.53 (q, $J=6.50$, $2 \mathrm{H}$ ), $2.93\left(\mathrm{t}, J=7.6 \mathrm{~Hz}, 2 \mathrm{H}\right.$ ), 2.11 (quin, $J=10.6 \mathrm{~Hz}, 2 \mathrm{H}$ ) (one $\mathrm{CH}_{2}$ merged in solvent peak). ${ }^{13} \mathrm{C}$ NMR not assigned. Purity: HPLC $t_{\mathrm{R}}=5.94 \mathrm{~min}$, HPLC purity $>99.0 \%$.

\section{5-(3,4-Difluorophenyl)-N-(3-(pyridin-2-yl)propyl)oxazole-4-carboxamide (35,} OCM-35).-The title compound was synthesized from 5-(3,4-difluorophenyl)oxazole-4carboxylic acid $(0.12 \mathrm{~g}, 0.53 \mathrm{mmol})$ and 3-(pyridin-2-yl)propan-1-amine $(0.087 \mathrm{~g}, 0.64$ mmol) to give $35(0.09 \mathrm{~g}, 49 \%)$ as an off-white solid. LCMS (ESI): $\mathrm{m} / \mathrm{z}=344.05[\mathrm{M}+\mathrm{H}]^{+}$. HRMS $\left(\mathrm{ESI}^{+}\right): \mathrm{m} / \mathrm{z}$ calcd for $\mathrm{C}_{18} \mathrm{H}_{16} \mathrm{~F}_{2} \mathrm{~N}_{3} \mathrm{O}_{2}{ }^{+}(\mathrm{M}+\mathrm{H})^{+}, 344.1211$; found, 344.1214. ${ }^{1} \mathrm{H}$ NMR (400 MHz, chloroform- $d$ ): $\delta 8.56(\mathrm{~d}, J=3.2 \mathrm{~Hz}, 1 \mathrm{H}), 8.40-8.34(\mathrm{~m}, 1 \mathrm{H}), 8.17-8.13$ (m, 1H), $7.85(\mathrm{~s}, 1 \mathrm{H}), 7.67$ (br s, 1H), $7.60(\mathrm{dt}, J=2.00,7.80 \mathrm{~Hz}, 1 \mathrm{H}), 7.26-7.11(\mathrm{~m}, 3 \mathrm{H})$, $3.52(\mathrm{q}, J=6.50 \mathrm{~Hz}, 2 \mathrm{H}), 2.93(\mathrm{t}, J=7.40 \mathrm{~Hz}, 2 \mathrm{H}), 2.18-2.06(\mathrm{~m}, 2 \mathrm{H}) .{ }^{13} \mathrm{C}$ NMR $(100$ MHz, DMSO- $\left.d_{6}\right): \delta 160.06,160.42,150.77(\mathrm{~m}), 150.41,148.91,148.29(\mathrm{~m}), 136.42$, 129.53, $124.92(\mathrm{~m}), 124.42(\mathrm{~m}), 122.69,121.17,117.84(\mathrm{~d}, J=17.7 \mathrm{~Hz}), 117.24(\mathrm{~d}, J=20.7$ $\mathrm{Hz}), 38.54,35.01,28.94$. Purity: $\mathrm{HPLC} t_{\mathrm{R}}=5.69 \mathrm{~min}$, HPLC purity $>96.6 \%$.

\section{5-(3,5-Difluoro-4-methoxyphenyl)-N-(3-(pyridin-2-yl)propyl)-oxazole-4-} carboxamide (36, OCM-36).-The title compound was synthesized from 5-(3,5difluoro-4-methoxyphenyl)oxazole-4-carboxylic acid $(0.140 \mathrm{~g}, 0.55 \mathrm{mmol})$ and 3(pyridin-2-yl)propan-1-amine (0.07 g, $0.55 \mathrm{mmol})$ to give $36(0.060 \mathrm{~g}, 29 \%)$ as an off-white solid after silica gel column purification. LCMS (ESI): $\mathrm{m} / z=374.10[\mathrm{M}+\mathrm{H}]^{+}$. HRMS (ESI $\left.{ }^{+}\right): \mathrm{m} / \mathrm{z}$ calcd for $\mathrm{C}_{19} \mathrm{H}_{18} \mathrm{~F}_{2} \mathrm{~N}_{3} \mathrm{O}_{3}{ }^{+}(\mathrm{M}+\mathrm{H})^{+}, 374.1316$; found, 374.1309. ${ }^{1} \mathrm{H}$ NMR (400 MHz, chloroform- $d$ ): $\delta 8.55(\mathrm{~d}, J=5.2 \mathrm{~Hz}, 1 \mathrm{H}), 8.12-8.02(\mathrm{~m}, 2 \mathrm{H}), 7.82(\mathrm{~s}, 1 \mathrm{H}), 7.66(\mathrm{br} \mathrm{s}$, $1 \mathrm{H}), 7.62-7.56(\mathrm{~m}, 1 \mathrm{H}), 7.18(\mathrm{~d}, J=7.60 \mathrm{~Hz}, 1 \mathrm{H}), 7.13-7.10(\mathrm{~m}, 1 \mathrm{H}), 4.07(\mathrm{~s}, 3 \mathrm{H}), 3.51(\mathrm{q}$, $J=6.50 \mathrm{~Hz}, 2 \mathrm{H}), 2.91(\mathrm{t}, J=7.40 \mathrm{~Hz}, 2 \mathrm{H}), 2.14-2.05(\mathrm{~m}, 2 \mathrm{H}) .{ }^{13} \mathrm{C} \mathrm{NMR}(100 \mathrm{MHz}$, $\left.\mathrm{CDCl}_{3}\right): \delta 161.02,160.80,155.04(\mathrm{dd}, J=246$ and $6.7 \mathrm{~Hz}), 150.27,149.28,147.84,137.74$ (m), 136.49, 129.44, 122.92, 121.41 (m), 121.19, 112.38 (m), 61.73, 38.94, 35.57, 29.13. Purity: HPLC $t_{\mathrm{R}}=5.81 \mathrm{~min}$, HPLC purity $>99.0 \%$.

\section{5-(3-Fluoro-4-methoxyphenyl)-N-(3-(pyridin-2-yl)propyl)-oxazole-4-}

carboxamide (37, OCM-37).-The title compound was synthesized from 5-(3-fluoro-4methoxyphenyl)oxazole-4-carboxylic acid (0.2 g, $0.84 \mathrm{mmol}$ ) and 3-(pyridin-2-yl)propan-1amine $(0.37 \mathrm{~g}, 1.00 \mathrm{mmol})$ to give $37(0.130 \mathrm{~g}, 44 \%)$, as an off-white solid. LCMS (ESI): $\mathrm{m} / \mathrm{z}=356.10[\mathrm{M}+\mathrm{H}]^{+}$. HRMS $\left(\mathrm{ESI}^{+}\right): \mathrm{m} / z$ calcd for $\mathrm{C}_{19} \mathrm{H}_{19} \mathrm{FN}_{3} \mathrm{O}_{3}{ }^{+}(\mathrm{M}+\mathrm{H})^{+}, 356.1410$; found, 356.1404. ${ }^{1} \mathrm{H}$ NMR (400 MHz, DMSO- $\left.d_{6}\right): \delta 8.54(\mathrm{~s}, 1 \mathrm{H}), 8.52-8.46(\mathrm{~m}, 2 \mathrm{H}), 8.35$ (dd, $J=2.00,13.60 \mathrm{~Hz}, 1 \mathrm{H}), 7.96(\mathrm{~d}, J=8.40 \mathrm{~Hz}, 1 \mathrm{H}), 7.68(\mathrm{dt}, J=1.60,7.60 \mathrm{~Hz}, 1 \mathrm{H})$, 7.32-7.26 (m, 2H), 7.20-7.17 (m, 1H), 3.90 (s, 3H), 3.34-3.29 (m, 2H), 2.78-2.74 (m, 2H), 1.92 (quin, $J=7.40 \mathrm{~Hz}, 2 \mathrm{H}) .{ }^{13} \mathrm{C}$ NMR $\left(100 \mathrm{MHz}\right.$, DMSO- $\left.d_{6}\right): \delta 161.09,160.65,150.78(\mathrm{~d}$, $J=241.3 \mathrm{~Hz}), 149.80,149.74,148.92,148.30$ (d, $J=10.6 \mathrm{~Hz}), 136.38,128.31,124.44$ (d, $J$ $=3 \mathrm{~Hz}), 122.67,121.15,119.68(\mathrm{~d}, J=8.1 \mathrm{~Hz}), 115.49(\mathrm{~d}, J=21.5 \mathrm{~Hz}), 113.63,56.10$, 38.48, 35.03, 29.01. Purity: HPLC $t_{\mathrm{R}}=5.51 \mathrm{~min}$, $\mathrm{HPLC}$ purity $>99.2 \%$. 
5-(3-Fluoro-4-(trifluoromethyl)phenyl)-N-(3-(pyridin-3-yl)-propyl)oxazole-4carboxamide (39, OCM-39).-The title compound was synthesized from 5-(3-fluoro-4(trifluoromethyl)phenyl)oxazole-4-carboxylic acid $(0.2 \mathrm{~g}, 0.72 \mathrm{mmol})$ and 3-(pyridin-3yl)propan-1-amine $(0.12 \mathrm{~g}, 0.87 \mathrm{mmol})$ to give $39(0.045 \mathrm{~g}, 15 \%)$ as an off-white solid. LCMS (ESI): $m / z=394.10[\mathrm{M}+\mathrm{H}]^{+}$. HRMS $\left(\mathrm{ESI}^{+}\right): \mathrm{m} / z$ calcd for $\mathrm{C}_{19} \mathrm{H}_{16} \mathrm{~F}_{4} \mathrm{~N}_{3} \mathrm{O}_{2}{ }^{+}(\mathrm{M}+$ $\mathrm{H})^{+}, 394.1179$; found, 394.1172. ${ }^{1} \mathrm{H}$ NMR (400 MHz, chloroform- $d$ ): $\delta 8.60$ (br s, $\left.1 \mathrm{H}\right), 8.39$ $(\mathrm{d}, J=11.6 \mathrm{~Hz}, 1 \mathrm{H}), 8.26(\mathrm{~d}, J=8 \mathrm{~Hz}, 1 \mathrm{H}), 7.92(\mathrm{~s}, 1 \mathrm{H}), 7.70(\mathrm{t}, J=8.00 \mathrm{~Hz}, 1 \mathrm{H}), 7.57(\mathrm{~d}, J$ $=8.00 \mathrm{~Hz}, 1 \mathrm{H}$ ), $7.42(\mathrm{~m}, 1 \mathrm{H}), 3.52(\mathrm{q}, J=6.8 \mathrm{~Hz}, 2 \mathrm{H}), 2.76(\mathrm{t}, J=7.8 \mathrm{~Hz}, 2 \mathrm{H}$ ), 2.00 (quin, $J$ $=7.40 \mathrm{~Hz}, 2 \mathrm{H})(1 \mathrm{H}$ merged in the solvent peak $) .{ }^{13} \mathrm{C}$ NMR not assigned. Purity: HPLC $t_{\mathrm{R}}=$ $6.34 \mathrm{~min}$, HPLC purity $>97.7 \%$.

N-(3-(Pyridin-3-yl)propyl)-5-(3,4,5-trifluorophenyl)oxazole-4-car-boxamide (40, OCM-40).-The title compound was synthesized from 5-(3,4,5-trifluorophenyl)oxazole-4carboxylic acid ( $0.2 \mathrm{~g}, 0.82 \mathrm{mmol})$ and 3-(pyridin-3-yl)propan-1-amine $(0.135 \mathrm{~g}, 0.98$ mmol) to give $40(0.070 \mathrm{~g}, 23 \%)$ as an off-white solid. LCMS (ESI): $\mathrm{m} / \mathrm{z}=362.65[\mathrm{M}+\mathrm{H}]$ ${ }^{+}$. HRMS $\left(\mathrm{ESI}^{+}\right): \mathrm{m} / \mathrm{z}$ calcd for $\mathrm{C}_{18} \mathrm{H}_{15} \mathrm{~F}_{3} \mathrm{~N}_{3} \mathrm{O}_{2}{ }^{+}(\mathrm{M}+\mathrm{H})^{+}, 362.1116$; found, 362.1116. ${ }^{1} \mathrm{H}$ NMR (400 MHz, chloroform- $d$ ): $\delta 8.50(\mathrm{~s}, 1 \mathrm{H}), 8.47(\mathrm{~d}, J=4.40 \mathrm{~Hz}, 1 \mathrm{H}), 8.25-8.17(\mathrm{~m}$, 2H), $7.87(\mathrm{~s}, 1 \mathrm{H}), 7.56(\mathrm{~d}, J=8.00 \mathrm{~Hz}, 1 \mathrm{H}), 7.40(\mathrm{~m}, 1 \mathrm{H}), 7.26-7.23(\mathrm{~m}, 1 \mathrm{H}), 3.52(\mathrm{q}, J=$ $6.90 \mathrm{~Hz}, 2 \mathrm{H}$ ), 2.76-2.73 (m, 2H), 2.00 (quin, $J=7.40 \mathrm{~Hz}, 2 \mathrm{H}) .{ }^{13} \mathrm{C}$ NMR not assigned. Purity: HPLC $t_{\mathrm{R}}=6.01 \mathrm{~min}$, HPLC purity $>99.6 \%$.

5-(3,4-Difluorophenyl)-N-(3-(pyridin-3-yl)propyl)oxazole-4-carboxamide (41, OCM-41).-The title compound was synthesized from 5-(3,4-difluorophenyl)oxazole-4carboxylic acid $(0.150 \mathrm{~g}, 0.66 \mathrm{mmol})$ and 3-(pyridin-3-yl)propan-1-amine $(0.11 \mathrm{~g}, 0.80$ mmol) to give $41(0.10 \mathrm{~g}, 43.5 \%)$ as an off white solid. LCMS (ESI): $\mathrm{m} / \mathrm{z}=344.05[\mathrm{M}+\mathrm{H}$ ] ${ }^{+}$. HRMS $\left(\mathrm{ESI}^{+}\right): \mathrm{m} / \mathrm{z}$ calcd for $\mathrm{C}_{18} \mathrm{H}_{16} \mathrm{~F}_{2} \mathrm{~N}_{3} \mathrm{O}_{2}{ }^{+}(\mathrm{M}+\mathrm{H})^{+}, 344.1211$; found, 344.1201. ${ }^{1} \mathrm{H}$ NMR (400 MHz, chloroform- $d$ ): $\delta 8.49-8.46(\mathrm{~m}, 2 \mathrm{H}), 8.39-8.34(\mathrm{~m}, 1 \mathrm{H}), 8.16-8.13(\mathrm{~m}$, $1 \mathrm{H}), 7.85(\mathrm{~s}, 1 \mathrm{H}), 7.54(\mathrm{~d}, J=8.00 \mathrm{~Hz}, 1 \mathrm{H}), 7.38(\mathrm{br} \mathrm{s}, 1 \mathrm{H}), 7.30-7.20(\mathrm{~m}, 2 \mathrm{H}), 3.51(\mathrm{q}, J=$ $6.70 \mathrm{~Hz}, 2 \mathrm{H}), 2.74(\mathrm{t}, J=7.80 \mathrm{~Hz}, 2 \mathrm{H}), 2.03-1.95(\mathrm{~m}, 2 \mathrm{H}) .{ }^{13} \mathrm{C}$ NMR not assigned. Purity: HPLC $t_{\mathrm{R}}=5.72 \mathrm{~min}, \mathrm{HPLC}$ purity $>97.3 \%$.

5-(3,5-Difluoro-4-methoxyphenyl)-N-(3-(pyridin-3-yl)propyl)-oxazole-4carboxamide (42, OCM-42).-The title compound was synthesized from 5-(3,5difluoro-4-methoxyphenyl)oxazole-4-carboxylic acid $(0.2 \mathrm{~g}, 0.78 \mathrm{mmol})$ and 3-(pyridin-3yl)propan-1-amine $(0.127 \mathrm{~g}, 0.93 \mathrm{mmol})$ to give $\mathbf{4 2}(0.1 \mathrm{~g}, 34.6 \%)$ as an off-white solid. LCMS (ESI): $\mathrm{m} / z=374.15[\mathrm{M}+\mathrm{H}]^{+}$. HRMS $\left(\mathrm{ESI}^{+}\right): \mathrm{m} / z$ calcd for $\mathrm{C}_{19} \mathrm{H}_{18} \mathrm{~F}_{2} \mathrm{~N}_{3} \mathrm{O}_{3}{ }^{+}(\mathrm{M}+$ $\mathrm{H})^{+}$, 374.1316; found, 374.1321. ${ }^{1} \mathrm{H}$ NMR (400 MHz, DMSO- $\left.d_{6}\right): \delta 8.61(\mathrm{~s}, 1 \mathrm{H}), 8.60-8.56$ (m, 1H), 8.50-8.35 (m, 2H), 8.18-8.06 (m, 2H), $7.64(\mathrm{~d}, J=7.60 \mathrm{~Hz}, 1 \mathrm{H}), 7.29(\mathrm{~m}, 1 \mathrm{H})$, $4.00(\mathrm{~s}, 3 \mathrm{H}), 3.31-3.26(\mathrm{~m}, 2 \mathrm{H}), 2.62(\mathrm{t}, J=7.60 \mathrm{~Hz}, 2 \mathrm{H}), 1.90-1.80(\mathrm{~m}, 2 \mathrm{H}) .{ }^{13} \mathrm{C}$ NMR $\left(100 \mathrm{MHz}\right.$, DMSO- $\left.d_{6}\right): \delta 160.42,154.39$ (dd, $J=244$ and $\left.6.7 \mathrm{~Hz}\right), 150.37,149.57,148.50$, 147.10, 136.96 (m), 136.79 (d, $J=13.9 \mathrm{~Hz}), 135.75,129.66,123.45,121.64(\mathrm{~m}), 112.09$ (m), 61.75, 38.29, 30.35, 29.60. Purity: HPLC $t_{\mathrm{R}}=5.90 \mathrm{~min}$, HPLC purity $>99.4 \%$.

5-(3-Fluoro-4-methoxyphenyl)-N-(3-(pyridin-3-yl)propyl)-oxazole-4carboxamide (44, OCM-44).-The title compound was synthesized from 5-(3-fluoro-4- 
methoxyphenyl)oxazole-4-carboxylic acid (0.2 g, $0.84 \mathrm{mmol}$ ) and 3-(pyridin-3-yl)propan-1amine $(0.137 \mathrm{~g}, 1.00 \mathrm{mmol})$ to give $\mathbf{4 4}(0.145 \mathrm{~g}, 48 \%)$ as an off-white solid. LCMS (ESI): $\mathrm{m} / \mathrm{z}=356.10[\mathrm{M}+\mathrm{H}]^{+}$. HRMS $\left(\mathrm{ESI}^{+}\right): \mathrm{m} / z$ calcd for $\mathrm{C}_{19} \mathrm{H}_{19} \mathrm{FN}_{3} \mathrm{O}_{3}{ }^{+}(\mathrm{M}+\mathrm{H})^{+}, 356.1410$; found, 356.1402. ${ }^{1} \mathrm{H}$ NMR (400 MHz, DMSO- $\left.d_{6}\right): \delta 8.55(\mathrm{~s}, 1 \mathrm{H}), 8.49(\mathrm{t}, J=5.60 \mathrm{~Hz}, 1 \mathrm{H})$, $8.45(\mathrm{~d}, J=2.00 \mathrm{~Hz}, 1 \mathrm{H}), 8.39$ (dd, $J=1.40,4.80 \mathrm{~Hz}, 1 \mathrm{H}), 8.35(\mathrm{dd}, J=2.20,13.20 \mathrm{~Hz}$, $1 \mathrm{H}), 7.97(\mathrm{~d}, J=8.40 \mathrm{~Hz}, 1 \mathrm{H}), 7.66-7.64(\mathrm{~m}, 1 \mathrm{H}), 7.32-7.28(\mathrm{~m}, 2 \mathrm{H}), 3.91(\mathrm{~s}, 3 \mathrm{H}), 3.34$ $3.26(\mathrm{~m}, 2 \mathrm{H}), 2.63(\mathrm{t}, J=7.80 \mathrm{~Hz}, 2 \mathrm{H}), 1.84$ (quin, $J=7.34 \mathrm{~Hz}, 2 \mathrm{H}) .{ }^{13} \mathrm{C} \mathrm{NMR}(100 \mathrm{MHz}$, DMSO- $\left.d_{6}\right): \delta 161.09,160.65,150.78(\mathrm{~d}, J=241.3 \mathrm{~Hz}), 149.80,149.74,148.92,148.30(\mathrm{~d}, J$ $=10.6 \mathrm{~Hz}), 136.38,128.31,124.44(\mathrm{~d}, J=3 \mathrm{~Hz}), 122.67,121.15,119.68(\mathrm{~d}, J=8.1 \mathrm{~Hz})$, $115.49(\mathrm{~d}, J=21.5 \mathrm{~Hz}), 113.64,56.08,38.20,30.38,29.58$. Purity: $\mathrm{HPLC} t_{\mathrm{R}}=5.55 \mathrm{~min}$, HPLC purity $>95.4 \%$.

\section{5-(3-Chloro-4-methoxyphenyl)-N-(3-(pyrazin-2-yl)propyl)-oxazole-4-} carboxamide (45, OCM-45).-The title compound was synthesized from 5-(3-chloro-4methoxyphenyl)oxazole-4-carboxylic acid $(0.12 \mathrm{~g}, 0.5 \mathrm{mmol})$ and 3-(pyrazin-2-yl)propan-1amine $(0.084 \mathrm{~g}, 0.61 \mathrm{mmol})$ to give $45(0.060 \mathrm{~g}, 31 \%)$ as an off-white solid. LCMS (ESI): $\mathrm{m} / \mathrm{z}=373.15[\mathrm{M}+\mathrm{H}]^{+}$. HRMS $\left(\mathrm{ESI}^{+}\right): \mathrm{m} / z$ calcd for $\mathrm{C}_{18} \mathrm{H}_{18} \mathrm{ClN}_{4} \mathrm{O}_{3}{ }^{+}(\mathrm{M}+\mathrm{H})^{+}, 373.1067$; found, 373.1064. ${ }^{1} \mathrm{H}$ NMR (400 MHz, chloroform- $d$ ): $\delta 8.51-8.50(\mathrm{~m}, 2 \mathrm{H}), 8.41(\mathrm{~d}, J=2.00$ $\mathrm{Hz}, 1 \mathrm{H}), 8.38(\mathrm{dd}, J=2.0,8.80 \mathrm{~Hz}, 1 \mathrm{H}), 8.33(\mathrm{~d}, J=2.00 \mathrm{~Hz}, 1 \mathrm{H}), 7.81(\mathrm{~s}, 1 \mathrm{H}), 7.48(\mathrm{br} \mathrm{s}$, $1 \mathrm{H}), 7.01(\mathrm{~d}, J=8.80 \mathrm{~Hz}, 1 \mathrm{H}), 3.96(\mathrm{~s}, 3 \mathrm{H}), 3.54(\mathrm{q}, J=6.80 \mathrm{~Hz}, 2 \mathrm{H}), 2.94(\mathrm{t}, J=7.60 \mathrm{~Hz}$, 2H), 2.16-2.09 (m, 2H). $\left.{ }^{13} \mathrm{C} \mathrm{NMR} \mathrm{(100} \mathrm{MHz,} \mathrm{CDCl}_{3}\right): \delta 161.12,156.71,156.16,151.45$, $147.52,144.60,144.01,142.36,129.82,128.44,128.11,122.45,120.36,111.53,56.21$, 38.66, 32.65, 28.84. Purity: $\mathrm{HPLC} t_{\mathrm{R}}=7.84 \mathrm{~min}$, $\mathrm{HPLC}$ purity $>99.8 \%$.

\section{5-(3-Chloro-4-methoxyphenyl)-N-(3-(pyridin-2-yl)propyl)-oxazole-4-} carboxamide (46, OCM-46).- The title compound was synthesized from 5-(3-chloro-4methoxyphenyl)oxazole-4-carboxylic acid $(0.2 \mathrm{~g}, 0.79 \mathrm{mmol})$ and 3-(pyridin-2-yl)propan-1amine $(0.13 \mathrm{~g}, 0.95 \mathrm{mmol})$ to give $\mathbf{4 6}(0.090 \mathrm{~g}, 30 \%)$ as an off-white solid. LCMS (ESI): $\mathrm{m} / \mathrm{z}=372.25[\mathrm{M}+\mathrm{H}]^{+}$. HRMS $\left(\mathrm{ESI}^{+}\right): \mathrm{m} / z$ calcd for $\mathrm{C}_{19} \mathrm{H}_{19} \mathrm{ClN}_{3} \mathrm{O}_{3}{ }^{+}(\mathrm{M}+\mathrm{H})^{+}, 372.1115$; found, 372.1116. ${ }^{1} \mathrm{H}$ NMR (400 MHz, DMSO- $d_{6}$ ): $\delta 8.53-8.45$ (m, 4H), 8.10 (dd, $J=2.00$, $8.60 \mathrm{~Hz}, 1 \mathrm{H}), 7.66(\mathrm{dt}, J=2.00,7.60 \mathrm{~Hz}, 1 \mathrm{H}), 7.28-7.25(\mathrm{~m}, 2 \mathrm{H}), 7.18-7.15(\mathrm{~m}, 1 \mathrm{H}), 3.91$ (s, 3H), 3.33-3.28 (m, 2H), $2.74(\mathrm{t}, J=7.40 \mathrm{~Hz}, 2 \mathrm{H}), 1.91$ (quin, $J=7.30 \mathrm{~Hz}, 2 \mathrm{H}) .{ }^{13} \mathrm{C}$ NMR (100 MHz, DMSO- $\left.d_{6}\right): \delta 161.10,160.63,155.47,149.83,149.65,148.94,136.40$, 129.31, 128.33, 127.91, 122.69, 121.17, 120.93, 120.30, 112.64, 56.31, 38.48, 35.04, 29.05. Purity: HPLC $t_{\mathrm{R}}=5.82 \mathrm{~min}$, HPLC purity $>98.8 \%$.

\section{5-(3-Chloro-4-methoxyphenyl)-N-(3-(pyridin-3-yl)propyl)-oxazole-4-} carboxamide (47, OCM-47).-The title compound was synthesized from 5-(3-chloro-4methoxyphenyl)oxazole-4-carboxylic acid $(0.2 \mathrm{~g}, 0.79 \mathrm{mmol})$ and 3-(pyridin-3-yl)propan-1amine $(0.13 \mathrm{~g}, 0.95 \mathrm{mmol})$ to give $47(0.12 \mathrm{~g}, 40.5 \%)$ as an off-white solid. LCMS (ESI): $\mathrm{m} / \mathrm{z}=372.20[\mathrm{M}+\mathrm{H}]^{+}$. HRMS $\left(\mathrm{ESI}^{+}\right): \mathrm{m} / z$ calcd for $\mathrm{C}_{19} \mathrm{H}_{19} \mathrm{ClN}_{3} \mathrm{O}_{3}{ }^{+}(\mathrm{M}+\mathrm{H})^{+}, 372.1115$; found, 372.1107. ${ }^{1} \mathrm{H}$ NMR (400 MHz, DMSO- $\left.d_{6}\right): \delta 8.55(\mathrm{~s}, 1 \mathrm{H}), 8.53(\mathrm{~d}, J=2.00 \mathrm{~Hz}, 1 \mathrm{H})$, $8.50-8.45(\mathrm{~m}, 2 \mathrm{H}), 8.39(\mathrm{~d}, J=3.60 \mathrm{~Hz}, 1 \mathrm{H}), 8.11(\mathrm{dd}, J=2.00,8.80 \mathrm{~Hz}, 1 \mathrm{H}), 7.65(\mathrm{~d}, J=$ $7.60 \mathrm{~Hz}, 1 \mathrm{H}), 7.31-7.28(\mathrm{~m}, 2 \mathrm{H}), 3.92(\mathrm{~s}, 3 \mathrm{H}), 3.26-3.27(\mathrm{~m}, 2 \mathrm{H}), 2.63(\mathrm{t}, J=7.80 \mathrm{~Hz}, 2 \mathrm{H})$, 1.84 (quin, $J=7.30 \mathrm{~Hz}, 2 \mathrm{H}$ ). ${ }^{13} \mathrm{C}$ NMR (100 MHz, DMSO- $\left.d_{6}\right)$ : $\delta=160.67,155.47,149.80$, 
$149.67,149.58,147.09,137.03,135.75,129.29,128.30,127.90,123.36,120.93,120.28$, $112.63,56.30,38.22,30.44,29.61$. Purity: HPLC $t_{\mathrm{R}}=5.88 \mathrm{~min}$, HPLC purity $>98.8 \%$.

5-(3-Chloro-4-methoxyphenyl)-N-(2-(5-fluoro-1H-indol-1-yl)-ethyl)oxazole-4carboxamide (48, OCM-48).- The title compound was synthesized from 5-(3-chloro-4methoxyphenyl)oxazole-4-carboxylic acid $(0.2 \mathrm{~g}, 0.79 \mathrm{mmol})$ and 2-(5-fluoro- $1 H$-indol-1yl)ethan-1-amine $(0.17 \mathrm{~g}, 0.95 \mathrm{mmol})$, and the crude residue obtained was purified by the Grace reverse phase system ( $40 \mathrm{~g} \mathrm{C}-18$ cartridge, gradient $0-70 \% \mathrm{ACN}$ in $\mathrm{H}_{2} \mathrm{O}$ ) and lyophilized to give $48(0.09 \mathrm{~g}, 28 \%)$ as an off-white solid. LCMS (ESI): $\mathrm{m} / \mathrm{z}=414.10[\mathrm{M}+$ $\mathrm{H}]^{+}$. HRMS $\left(\mathrm{ESI}^{+}\right): \mathrm{m} / z$ calcd for $\mathrm{C}_{21} \mathrm{H}_{18} \mathrm{ClFN}_{3} \mathrm{O}_{3}{ }^{+}(\mathrm{M}+\mathrm{H})^{+}, 414.1021$; found, 414.1009. ${ }^{1} \mathrm{H}$ NMR (400 MHz, chloroform- $d$ ): $\delta 8.37-8.32(\mathrm{~m}, 2 \mathrm{H}), 7.75(\mathrm{~s}, 1 \mathrm{H}), 7.34-7.28(\mathrm{~m}, 2 \mathrm{H})$, $7.15(\mathrm{~d}, J=3.42 \mathrm{~Hz}, 1 \mathrm{H}), 7.04$ (d, $J=9.29 \mathrm{~Hz}, 1 \mathrm{H}), 6.97-6.91(\mathrm{~m}, 1 \mathrm{H}), 6.47$ (d, $J=2.93 \mathrm{~Hz}$, $1 \mathrm{H}), 4.37$ (t, $J=6.11 \mathrm{~Hz}, 2 \mathrm{H}), 3.98(\mathrm{~s}, 3 \mathrm{H}), 3.81(\mathrm{q}, J=6.20 \mathrm{~Hz}, 2 \mathrm{H}) .{ }^{13} \mathrm{C}$ NMR $(100 \mathrm{MHz}$, chloroform- $d$ ): $\delta 161.5,157.9(\mathrm{~d}, J=234 \mathrm{~Hz}), 156.3,151.8,147.6$ (d, $J=3.82 \mathrm{~Hz}), 132.6$, $129.9,129.5,128.9(\mathrm{~d}, J=10.60 \mathrm{~Hz}), 128.4,127.7,122.6,120.2,111.6,110.1(\mathrm{~d}, J=26$ Hz), $109.9(\mathrm{~d}, J=26 \mathrm{~Hz}), 105.8(\mathrm{~d}, J=9 \mathrm{~Hz}), 101.7,56.3,46.0$, 39.6. Purity: HPLC $t_{\mathrm{R}}=$ 8.99 min, HPLC purity $>98.8 \%$.

5-(3-Chloro-4-methoxyphenyl)-N-(3-(5-fluoropyridin-3-yl)-propyl)oxazole-4carboxamide (49, OCM-49).-The title compound was synthesized from 5-(3-chloro-4methoxyphenyl)oxazole-4-carboxylic acid $(0.1 \mathrm{~g}, 0.39 \mathrm{mmol})$ and 3-(5-fluoropyridin-3yl)propan-1-amine $(0.07 \mathrm{~g}, 0.47 \mathrm{mmol})$, and the crude residue obtained was purified by column chromatography (230-400 mesh silica gel gradient 1-50\% EtOAc in $n$-hexane) to give $49(0.077 \mathrm{~g}, 50 \%)$ as an off-white solid. LCMS (ESI): $\mathrm{m} / \mathrm{z}=390.05[\mathrm{M}+\mathrm{H}]^{+}$. HRMS $\left(\mathrm{ESI}^{+}\right): \mathrm{m} / \mathrm{z}$ calcd for $\mathrm{C}_{19} \mathrm{H}_{18} \mathrm{ClFN}_{3} \mathrm{O}_{3}{ }^{+}(\mathrm{M}+\mathrm{H})^{+}, 390.1021$; found, 390.1026. ${ }^{1} \mathrm{H}$ NMR $(400 \mathrm{MHz}$, chloroform- $d$ ): $\delta 8.37$ (dd, $J=2.20,8.56 \mathrm{~Hz}, 1 \mathrm{H}), 8.35-8.28(\mathrm{~m}, 3 \mathrm{H}), 7.80(\mathrm{~s}$, 1H), 7.37-7.27 (m, 2H), 7.01 (d, $J=8.80 \mathrm{~Hz}, 1 \mathrm{H}), 3.96$ (s, 3H), 3.51 (q, $J=6.68 \mathrm{~Hz}, 2 \mathrm{H}$ ), $2.75(\mathrm{t}, J=7.83 \mathrm{~Hz}, 2 \mathrm{H}), 2.04-1.96(\mathrm{~m}, 2 \mathrm{H}) .{ }^{13} \mathrm{C} \mathrm{NMR}$ (100 MHz, chloroform- $d$ ): $\delta 161.1$, $159.46(\mathrm{~d}, J=255 \mathrm{~Hz}), 156.2,151.5,147.5(\mathrm{~d}, J=4 \mathrm{~Hz}), 145.6(\mathrm{~d}, J=3 \mathrm{~Hz}), 138.5(\mathrm{~d}, J=4$ $\mathrm{Hz}), 135.9$ (d, $J=23 \mathrm{~Hz}), 129.8,128.4,128.0,122.5$ (d, $J=17 \mathrm{~Hz}), 122.4,120.3,111.5$, $56.2,38.6,30.8,29.9$. Purity: HPLC $t_{\mathrm{R}}=8.61 \mathrm{~min}$, HPLC purity $>99.3 \%$.

5-(3-Chloro-4-methoxyphenyl)-N-(3-(6-fluoropyridin-3-yl)-propyl)oxazole-4carboxamide (50, OCM-50).-The title compound was synthesized from 5-(3-chloro-4methoxyphenyl)oxazole-4-carboxylic acid $(0.1 \mathrm{~g}, 0.39 \mathrm{mmol})$ and 3-(6-fluoropyridin-3yl)propan-1-amine $(0.07 \mathrm{~g}, 0.47 \mathrm{mmol})$, and the crude residue obtained was purified by column chromatography (gradient $0-70 \%$ EtOAc in $n$-hexane) to give $\mathbf{5 0}(0.05 \mathrm{~g}, 33 \%)$ as an off-white solid. LCMS (ESI): $\mathrm{m} / z=390.05[\mathrm{M}+\mathrm{H}]^{+}$. HRMS $\left(\mathrm{ESI}^{+}\right): \mathrm{m} / \mathrm{z}$ calcd for $\mathrm{C}_{19} \mathrm{H}_{18} \mathrm{ClFN}_{3} \mathrm{O}_{3}{ }^{+}(\mathrm{M}+\mathrm{H})^{+}$, 390.1021; found, 390.1015. ${ }^{1} \mathrm{H}$ NMR (400 MHz, chloroformd): $\delta 8.38(\mathrm{dd}, J=1.96,8.80 \mathrm{~Hz}, 1 \mathrm{H}), 8.35(\mathrm{~d}, J=1.96 \mathrm{~Hz}, 1 \mathrm{H}), 8.07(\mathrm{~s}, 1 \mathrm{H}), 7.82(\mathrm{~s}, 1 \mathrm{H})$, 7.67-7.62 (m, 1H), $7.34(\mathrm{br} \mathrm{s}, 1 \mathrm{H}), 7.02(\mathrm{~d}, J=8.80 \mathrm{~Hz}, 1 \mathrm{H}), 6.87(\mathrm{dd}, J=2.45,8.31 \mathrm{~Hz}$, $1 \mathrm{H}), 3.97(\mathrm{~s}, 3 \mathrm{H}), 3.51(\mathrm{q}, J=6.85 \mathrm{~Hz}, 2 \mathrm{H}), 2.73(\mathrm{t}, J=7.83 \mathrm{~Hz}, 2 \mathrm{H}), 2.01-1.93(\mathrm{~m}, 2 \mathrm{H})$. ${ }^{13} \mathrm{C}$ NMR (100 MHz, chloroform- $d$ ): $\delta 162.5$ (d, $\left.J=237.6 \mathrm{~Hz}\right), 161.2,156.3,151.6,147.6$ (d, $J=4.24 \mathrm{~Hz}), 147.0(\mathrm{~d}, J=12.29 \mathrm{~Hz}), 141.0(\mathrm{~d}, J=7.21 \mathrm{~Hz}), 134.2(\mathrm{~d}, J=4.24 \mathrm{~Hz})$, 
$129.9,128.5,128.0,122.5,120.3,111.6,109.3(\mathrm{~d}, J=37.1 \mathrm{~Hz}), 56.2(\mathrm{~d}, J=3.39 \mathrm{~Hz}), 38.6$, 31.1, 29.5. Purity: HPLC $t_{\mathrm{R}}=8.91 \mathrm{~min}$, HPLC purity $>99.8 \%$.

N-(3-(5-Fluoropyridin-3-yl)propyl)-5-(3-iodo-4-methoxyphenyl)-oxazole-4carboxamide (51, OCM-51).-The title compound was synthesized from 5-(3-chloro-4methoxyphenyl)oxazole-4-carboxylic acid $(0.10 \mathrm{~g}, 0.29 \mathrm{mmol})$, and the crude residue obtained was purified by column chromatography (gradient 0-70\% EtOAc in $n$-hexane) to give $51(0.041 \mathrm{~g}, 29 \%)$ as an off-white solid. LCMS (ESI): $\mathrm{m} / \mathrm{z}=482.00[\mathrm{M}+\mathrm{H}]^{+}$. HRMS $\left(\mathrm{ESI}^{+}\right): \mathrm{m} / \mathrm{z}$ calcd for $\mathrm{C}_{19} \mathrm{H}_{18} \mathrm{FIN}_{3} \mathrm{O}_{3}{ }^{+}(\mathrm{M}+\mathrm{H})^{+}, 482.0377$; found, 482.0370. ${ }^{1} \mathrm{H}$ NMR (400 $\mathrm{MHz}$, chloroform- $d$ ): $\delta 8.64(\mathrm{~d}, J=1.96 \mathrm{~Hz}, 1 \mathrm{H}), 8.50(\mathrm{dd}, J=1.96,8.80 \mathrm{~Hz}, 1 \mathrm{H}), 8.33-$ 8.29 (m, 2H), $7.80(\mathrm{~s}, 1 \mathrm{H}), 7.33$ (br s, $1 \mathrm{H}), 7.28(\mathrm{br} \mathrm{s}, 1 \mathrm{H}), 6.90(\mathrm{~d}, J=8.80 \mathrm{~Hz}, 1 \mathrm{H}), 3.94(\mathrm{~s}$, $3 \mathrm{H}), 3.51$ (q, $J=6.85 \mathrm{~Hz}, 2 \mathrm{H}), 2.75(\mathrm{t}, J=7.83 \mathrm{~Hz}, 2 \mathrm{H}), 2.02-1.93(\mathrm{~m}, 2 \mathrm{H}) .{ }^{13} \mathrm{C}$ NMR $(100$ MHz, chloroform- $d$ ): $\delta 160.7,159.5(\mathrm{~d}, J=255.0 \mathrm{~Hz}), 159.3,150.8,147.1(\mathrm{~d}, J=4.66 \mathrm{~Hz})$, $145.2,138.4,138.1$ (d, $J=3.39 \mathrm{~Hz}), 135.8(\mathrm{~d}, J=23.2 \mathrm{~Hz}), 129.9,127.4,122.5(\mathrm{~d}, J=17.70$ $\mathrm{Hz}), 120.9,109.8,85.1,56.0,38.2,30.4,29.5$. Purity: HPLC $t_{\mathrm{R}}=8.94 \mathrm{~min}, \mathrm{HPLC}$ purity > $99.8 \%$.

\section{Radioligand Saturation Binding Assay.}

[ $\left.{ }^{3} \mathrm{H}\right] 1$ (81 Ci/mmol (3.0 TBq/mmol) molar activity, $99.9 \%$ radiochemical purity) was provided by MedChem Imaging, Inc. For additional details, see Supporting Information Materials and Methods.

\section{HEK-293T P301S hTau cellular Assay.}

HEK-293T P301S hTau cellular assay was performed as described in the Supporting Information Materials and Methods.

\section{In Vitro Autoradiography.}

For autoradiography experiments, fresh-frozen postmortem human brain tissue blocks were acquired from Folio-Conversant and the University of California at San Francisco NDBB. In vitro autoradiography studies were performed as described in the Supporting Information Materials and Methods. Phosphorimaging of tissue sections was performed using Amersham Typhoon (GE Healthcare, MA).

\section{Docking Studies.}

Molecular docking simulations of $\mathbf{4 4}$ and $\mathbf{5 1}$ were performed using the X-ray cocrystal structure of GSK- $3 \beta$ complexed with $\mathbf{1}$ (PDB code: $5 \mathrm{~K} 5 \mathrm{~N}$ ) and GSK- $3 \beta$ complexed with BRD0209 (PDB code: 5KPK) using the FITTED 3.5 program (FORECASTER platform). ${ }^{49-51}$ Docking structures and figures were prepared using Pymol.

\section{Kinase Assays.}

ADPGlo binding studies were performed as previously described. ${ }^{28}$ In vitro radiometric kinase assay including $K_{\mathrm{i}}$ determination and kinome selectivity profiles for selected inhibitors was obtained through $\left[\gamma^{33} \mathrm{P}\right]$ ATP-based enzymatic assays (Reaction Biology Corporation, Malvern, PA) ${ }^{30}$ For additional details, see the Supporting Information 
Materials and Methods. Detailed results for kinome selectivity can also be found in Tables S5-S7. Chemical proteomic (KiNativ) profiling using brain lysate was performed by ActivX Bioscience (La Jolla, CA). Detailed results for the kinativ profiling can be found in Table S8.

\section{CNS Target-Binding Assays.}

Inhibitor $\mathbf{5 1}$ was tested against a panel of 80 binding assays encompassing common targets found in the CNS (LeadProfilingScreen2 Panel, Eurofins Scientific). Detailed results for the $\mathrm{CNS}$ target-binding assay profiling are found in Table $\mathrm{S} 9$. The $\mathrm{IC}_{50}$ s for the five main offtarget of $51\left(5-\mathrm{HT}_{2 \mathrm{~B}}, \mathrm{BZD}, \mathrm{Cl}^{-}\right.$channel (GABA-gated), KOP, NK1) were determined using radio-ligand-binding assays (Eurofins Scientific). $K_{\mathrm{i}}$ values were calculated using the Cheng-Prusoff equation.

\section{MST Assay.}

The MST assay was performed at the Center for Macromolecule Interaction, Harvard University. For additional details, see the Supporting Information Materials and Methods.

\section{hERG Assay.}

hERG assays were performed at Reaction Biology Corporation (Malvern, PA) as previously described.

\section{WNT Reporter Assay.}

The WNT reporter assay was performed as described in the Supporting Information Materials and Methods and as previously described. ${ }^{41}$

\section{NPC Proliferation/Viability Assay.}

The NPC proliferation/viability assay was performed as described in the Supporting Information Materials and Methods.

\section{CRMP2 and pTau Assays.}

The CRMP2 and pTau assays were performed as described in Supporting Information Materials and Methods and as previously described. ${ }^{38,40}$

\section{Western Blot Analysis.}

The Western blot analysis was performed as described in the Supporting Information Materials and Methods.

\section{Radiochemical Syntheses (44).}

Detailed synthetic procedures for all radiotracers are provided in Scheme $\mathrm{S} 4$ and Supporting Information Materials and Methods. Briefly, for the radiosynthesis of $\left[{ }^{11} \mathrm{C}\right] 44,\left[{ }^{11} \mathrm{C}^{1} \mathrm{CH}_{3} \mathrm{OTf}\right.$ (obtained from $\sim 1000 \mathrm{mCi}$ of $\left[{ }^{11} \mathrm{C}^{2} \mathrm{CH}_{3} \mathrm{I}\right.$ ) was bubbled through a reaction solution containing $1.0 \mathrm{mg}$ of the corresponding desmethyl precursor, $5.0 \mu \mathrm{L}$ of $1.0 \mathrm{M}$ methanolic $\mathrm{TBAOH}$, and $100 \mu \mathrm{L}$ of DMF at room temperature for $3 \mathrm{~min}$ at $15 \mathrm{~mL} \mathrm{~min}^{-1}$ with Ar carrier gas. The reaction was quenched with $1000 \mu \mathrm{L} 50 / 50 \mathrm{MeCN}_{10} \mathrm{mM} \mathrm{NH}_{4} \mathrm{OAc}(\mathrm{pH}:$ 7.3) and 
loaded onto HPLC (50/50 MeCN/20 mM NH $4 \mathrm{OAc}(\mathrm{pH}: 7.3)$ (v/v) isocratic; flow rate: 5.0 $\mathrm{mL} / \mathrm{min}$; semipreparative column: Phenomenex Luna C18, $10 \mu \mathrm{m}, 10 \times 250 \mathrm{~mm}, 5 \mathrm{~mL} /$ $\mathrm{min}$ ). The peak between 7.5 and $8.1 \mathrm{~min}$ corresponding to $\left[{ }^{11} \mathrm{C}\right] \mathbf{4 4}$ (Figure S16) was collected and diluted with $50 \mathrm{~mL} \mathrm{H}_{2} \mathrm{O}$, and the solution was transferred through preconditioned C18 1cc Vac Sep Pak. The radiotracer was eluted with $500 \mu \mathrm{L}$ EtOH. The C18 cartridge was then washed with $4.5 \mathrm{~mL}$ buffered saline. Then, the product was passed through a sterile filter and into the product vial. Pure $\left[{ }^{11} \mathrm{C}\right] \mathbf{4 4}$ was obtained in $\sim 1 \%$ nondecay-corrected radiochemical yields at end-of-synthesis based upon $\left[{ }^{11} \mathrm{C}^{1} \mathrm{CH}_{3} \mathrm{I}\right.$, radiochemical purity $>99 \%$ determined by radio-HPLC, $A_{\mathrm{m}}=264 \mathrm{GBq} / \mu \mathrm{mol}(\mathrm{NHP}$ experiment), and $A_{\mathrm{m}}=426 \mathrm{GBq} / \mu \mathrm{mol}$ (rodent experiment). Quality control mobile phase: 50/50 MeCN/10mM NH $4 \mathrm{OAc}(\mathrm{pH}: 7.3)(\mathrm{v} / \mathrm{v})$ isocratic; flow rate: $1.0 \mathrm{~mL} / \mathrm{min}$; QC column: Phenomenex Luna C18, $5 \mu \mathrm{m}, 4.6 \times 150 \mathrm{~mm}$ (Figures S17 and S18).

\section{Rodents and Nonhuman Primate PET Imaging Studies (1, 28, 37, 44).}

All imaging studies were performed in accordance with the standards set by the University Committee on Use and Care of Animals (UCUCA) at the University of Michigan. Rat imaging was done using Sprague Dawley (body weight $\approx 297-339 \mathrm{~g}$ ). NHP imaging was done using a mature female rhesus monkey (Macaca mulatta) (body weight $\approx 8.8 \mathrm{~kg}$ with negligible variation throughout the duration of the study, 15 years of age). Imaging procedure and analysis is described in the Supporting Information Materials and Methods.

\section{Neuropharmacokinetic Analysis.}

NeuroPK was performed at Sai Life Sciences as described in the Supporting Information Materials and Methods.

\section{Statistical Analysis.}

Data were expressed as mean \pm SD. Statistical analysis was performed using GraphPad Prism 7.0c. $p$ values less than 0.05 were considered statistically significant.

\section{Supplementary Material}

Refer to Web version on PubMed Central for supplementary material.

\section{ACKNOWLEDGMENTS}

We thank Dr. Kelly L. Arnett and Harvard University's Center for Macromolecular Interaction for advice regarding MST and Dr. Poguang Wang for HRMS analyses. Funding to S.J.H. provided by the Tau Consortium and MGH Research Scholars Program. We thank Smita Jacob for graphic design. N.V. thanks National Institute on Ageing of the NIH (R01AG054473), the Azrieli Foundation and the Canada Research Chairs Program for support.

\section{ABBREVIATIONS}

\section{AD}

ADME

ADP
Alzheimer's disease

absorption; distribution, metabolism and elimination

adenosine $5^{\prime}$-diphosphate 


\begin{tabular}{|c|c|}
\hline AKT & protein kinase $\mathrm{B}$ \\
\hline$A_{\mathbf{m}}$ & molar activity \\
\hline APP & amyloid precursor protein \\
\hline Ar & argon \\
\hline ATP & adenosine $5^{\prime}$-triphosphate \\
\hline AUClast & area under time-concentration curve to last measurable concentration \\
\hline BBB & blood-brain barrier \\
\hline$B_{\max }$ & binding site density \\
\hline BZD & benzodiazepine \\
\hline CB & cerebellum \\
\hline $\mathbf{C C}$ & corpus callosum \\
\hline Cl & chloro \\
\hline$C_{\max }$ & maximum serum concentration \\
\hline CNS & central nervous system \\
\hline $\mathrm{CPu}$ & caudate putamen \\
\hline CRMP & collapsing response mediator protein \\
\hline Ctx & cortex \\
\hline DMF & dimethylformamide \\
\hline ESI & electrospray ionization \\
\hline EtOH & ethanol \\
\hline Fu_p & unbound brain fraction \\
\hline GABA & $\gamma$-aminobutyric acid \\
\hline GBq & gigabecquerel \\
\hline GM & grey matter \\
\hline GSK-3 & glycogen synthase kinase- 3 \\
\hline hERG & human ether-a-go-go related gene \\
\hline HP & hippocampus \\
\hline HT & hydroxytryptamine \\
\hline $\mathbf{I C}_{\mathbf{5 0}}$ & half-maximum inhibitory concentration \\
\hline
\end{tabular}




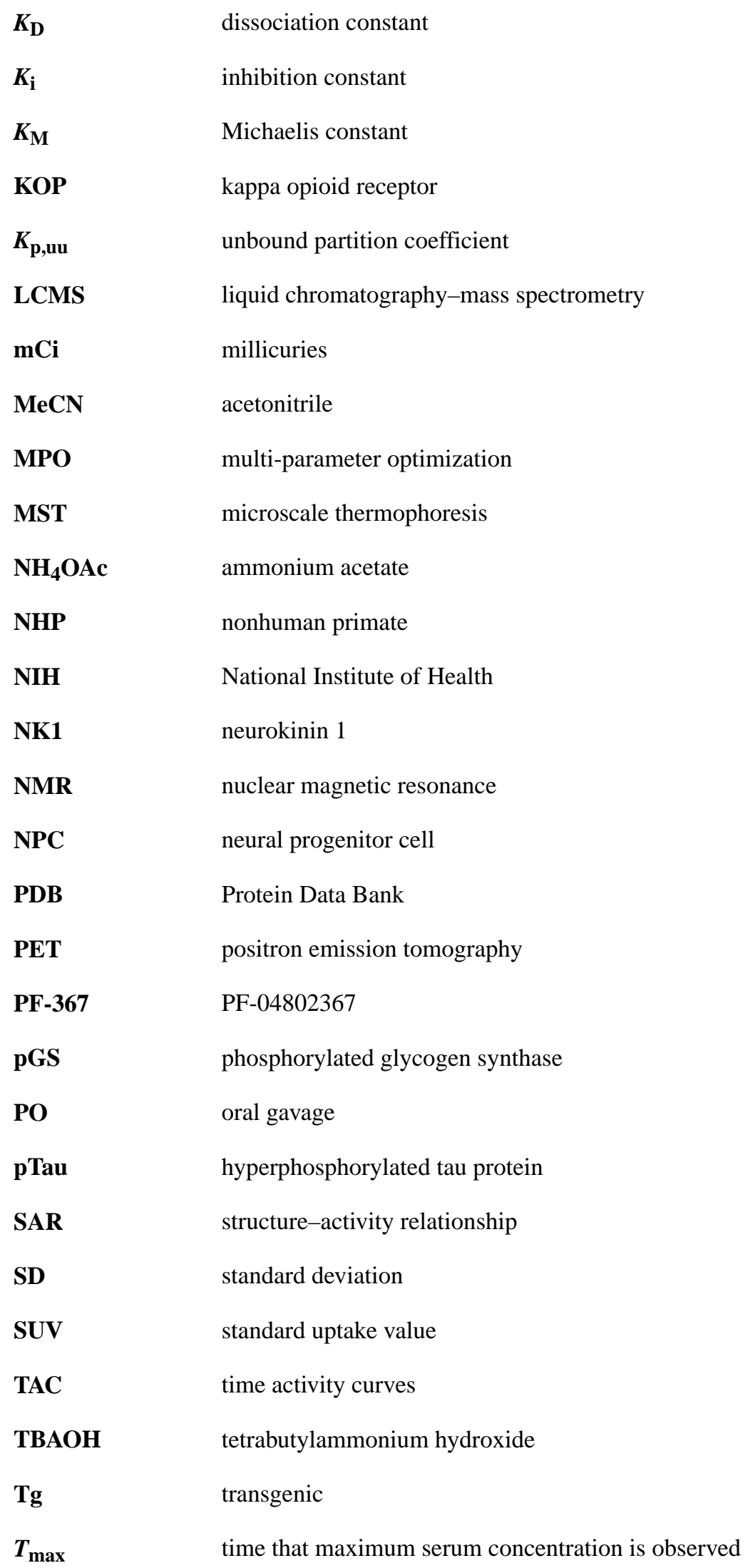




\section{UCUCA University Committee on Use and Care of Animals}

WM white matter

\section{REFERENCES}

(1). Cohen P; Frame S The renaissance of GSK3. Nat. Rev. Mol. Cell Biol. 2001, 2, 769-776. [PubMed: 11584304]

(2). Hur E-M; Zhou F-Q GSK3 signalling in neural development. Nat. Neurosci 2010, 11, 539-551.

(3). Sutherland C What Are thebona fideGSK3 Substrates? Int. J. Alzheimers Dis. 2011, 2011, 1-23.

(4). Cross DAE; Alessi DR; Cohen P; Andjelkovich M; Hemmings BA Inhibition of glycogen synthase kinase-3 by insulin mediated by protein kinase B. Nature 1995, 378, 785-789. [PubMed: 8524413]

(5). Eldar-Finkelman H; Seger R; Vandenheede JR; Krebs EG Inactivation of glycogen synthase kinase- 3 by epidermal growth factor is mediated by mitogen-activated protein kinase/p90 ribosomal protein S6 kinase signaling pathway in NIH/3T3 cells. J. Biol. Chem 1995, 270, 987 990. [PubMed: 7836418]

(6). Woodgett JR Molecular cloning and expression of glycogen synthase kinase-3/factor A. EMBO J. 1990, 9, 2431-2438. [PubMed: 2164470]

(7). Patel P; Woodgett JR Glycogen Synthase Kinase 3: A Kinase for All Pathways? Curr. Top. Dev. Biol 2017, 123, 277-302. [PubMed: 28236969]

(8). Hoeflich KP; Luo J; Rubie EA; Tsao M-S; Jin O; Woodgett JR Requirement for glycogen synthase kinase-3 $\beta$ in cell survival and NF- $\kappa$ B activation. Nature 2000, 406, 86-90. [PubMed: 10894547]

(9). Doble BW; Patel S; Wood GA; Kockeritz LK; Woodgett JR Functional Redundancy of GSK-3a and GSK-3 $\beta$ in Wnt/ $\beta$-Catenin Signaling Shown by Using an Allelic Series of Embryonic Stem Cell Lines. Dev. Cell 2007, 12, 957-971. [PubMed: 17543867]

(10). Hooper C; Killick R; Lovestone S The GSK3 hypothesis of Alzheimer's disease. J. Neurochem 2008, 104, 1433-1439. [PubMed: 18088381]

(11). Ballatore C; Lee VM-Y; Trojanowski JQ Tau-mediated neurodegeneration in Alzheimer's disease and related disorders. Nat. Rev. Neurosci 2007, 8, 663-672. [PubMed: 17684513]

(12). Lei P; Ayton S; Bush AI; Adlard PA GSK-3 in neurodegenerative diseases. Int. J. Alzheimers Dis. 2011, 2011, 1-9.

(13). Phiel CJ; Wilson CA; Lee VM-Y; Klein PS GSK-3 a regulates production of Alzheimer's disease amyloid- $\beta$ peptides. Nature 2003, 423, 435-439. [PubMed: 12761548]

(14). Ryder J; Su Y; Liu F; Li B; Zhou Y; Ni B Divergent roles of GSK3 and CDK5 in APP processing. Biochem. Biophys. Res. Commun 2003, 312, 922-929. [PubMed: 14651959]

(15). Klementiev B; Novikova T; Novitskaya V; Walmod PS; Dmytriyeva O; Pakkenberg B; Berezin V; Bock E A neural cell adhesion molecule-derived peptide reduces neuropathological signs and cognitive impairment induced by A $\beta 25-35$. Neuroscience 2007, 145, 209-224. [PubMed: 17223274]

(16). Hanger DP; Hughes K; Woodgett JR; Brion J-P; Anderton BH Glycogen synthase kinase-3 induces Alzheimer's disease-like phosphorylation of tau: generation of paired helical filament epitopes and neuronal localisation of the kinase. Neurosci. Lett 1992, 147, 58-62. [PubMed: 1336152]

(17). Ishiguro K; Omori A; Takamatsu M; Sato K; Arioka M; Uchida T; Imahori K Phosphorylation sites on tau by tau protein kinase I, a bovine derived kinase generating an epitope of paired helical filaments. Neurosci. Lett 1992, 148, 202-206. [PubMed: 1284442]

(18). Li C; Götz J Tau-based therapies in neurodegeneration: opportunities and challenges. Nat. Rev. Drug Discovery 2017, 16, 863-883. [PubMed: 28983098]

(19). Noble W; Planel E; Zehr C; Olm V; Meyerson J; Suleman F; Gaynor K; Wang L; LaFrancois J; Feinstein B; Burns M; Krishnamurthy P; Wen Y; Bhat R; Lewis J; Dickson D; Duff K Inhibition of glycogen synthase kinase- 3 by lithium correlates with reduced tauopathy and degeneration in vivo. Proc. Natl. Acad. Sci. U.S.A. 2005, 102, 6990-6995. [PubMed: 15867159] 
(20). Lucas JJ; Hernández F; Gómez-Ramos P; Morán MA; Hen R; Avila J Decreased nuclear betacatenin, tau hyper-phosphorylation and neurodegeneration in GSK-3beta conditional transgenic mice. EMBO J. 2001, 20, 27-39. [PubMed: 11226152]

(21). Pei J-J; Tanaka T; Tung Y-C; Braak E; Iqbal K; Grundke-iqbal I Distribution, levels, and activity of glycogen synthase kinase-3 in the Alzheimer disease brain. J. Neuropathol. Exp. Neurol 1997, 56, 70-78. [PubMed: 8990130]

(22). Blalock EM; Geddes JW; Chen KC; Porter NM; Markesbery WR; Landfield PW Incipient Alzheimer's disease: Microarray correlation analyses reveal major transcriptional and tumor suppressor responses. Proc. Natl. Acad. Sci. U.S.A. 2004, 101, 2173-2178. [PubMed: 14769913]

(23). Leroy K; Yilmaz Z; Brion JP Increased level of active GSK-3beta in Alzheimer's disease and accumulation in argyrophilic grains and in neurones at different stages of neurofibrillary degeneration. Neuropathol. Appl. Neurobiol 2007, 33, 43-55. [PubMed: 17239007]

(24). Kremer A; Louis JV; Jaworski T; Van Leuven F GSK3 and Alzheimer's disease: Facts and fiction... Front. Mol. Neurosci 2011, 4, 17. [PubMed: 21904524]

(25). Pandey MK; DeGrado TR Glycogen synthase kinase-3 (GSK-3)-targeted therapy and imaging. Theranostics 2016, 6, 571-593. [PubMed: 26941849]

(26). Cohen P; Goedert M GSK3 inhibitors: development and therapeutic potential. Nat. Rev. Drug Discovery 2004, 3, 479-487. [PubMed: 15173837]

(27). Wagner FF; Bishop JA; Gale JP; Shi X; Walk M; Ketterman J; Patnaik D; Barker D; Walpita D; Campbell AJ; Nguyen S; Lewis M; Ross L; Weïwer M; An WF; Germain AR; Nag PP; Metkar S; Kaya T; Dandapani S; Olson DE; Barbe A-L; Lazzaro F; Sacher JR; Cheah JH; Fei D; Perez J; Munoz B; Palmer M; Stegmaier K; Schreiber SL; Scolnick E; Zhang Y-L; Haggarty SJ; Holson EB; Pan JQ Inhibitors of Glycogen Synthase Kinase 3 with Exquisite Kinome-Wide Selectivity and Their Functional Effects. ACS Chem. Biol 2016, 11, 1952-1963. [PubMed: 27128528]

(28). Liang SH; Chen JM; Normandin MD; Chang JS; Chang GC; Taylor CK; Trapa P; Plummer MS; Para KS; Conn EL; Lopresti-Morrow L; Lanyon LF; Cook JM; Richter KEG; Nolan CE; Schachter JB; Janat F; Che Y; Shanmugasundaram V; Lefker BA; Enerson BE; Livni E; Wang L; Guehl NJ; Patnaik D; Wagner FF; Perlis R; Holson EB; Haggarty SJ; El Fakhri G; Kurumbail RG; Vasdev N Discovery of a highly selective glycogen synthase kinase-3 inhibitor (PF-04802367) that modulates tau phosphorylation in the brain: translation for PET neuroimaging. Angew. Chem., Int. Ed. Engl 2016, 55, 9601-9605. [PubMed: 27355874]

(29). Luo G; Chen L; Burton CR; Xiao H; Sivaprakasam P; Krause CM; Cao Y; Liu N; Lippy J; Clarke WJ; Snow K; Raybon J; Arora V; Pokross M; Kish K; Lewis HA; Langley DR; Macor JE; Dubowchik GM Discovery of isonicotinamides as highly selective, brain penetrable, and orally active glycogen synthase kinase-3 inhibitors. J. Med. Chem 2016, 59, 1041-1051. [PubMed: 26751161]

(30). Anastassiadis T; Deacon SW; Devarajan K; Ma H; Peterson JR Comprehensive assay of kinase catalytic activity reveals features of kinase inhibitor selectivity. Nat. Biotechnol 2011, 29, 1039 1045. [PubMed: 22037377]

(31). Davis MI; Hunt JP; Herrgard S; Ciceri P; Wodicka LM; Pallares G; Hocker M; Treiber DK; Zarrinkar PP Comprehensive analysis of kinase inhibitor selectivity. Nat. Biotechnol 2011, 29, 1046-1051. [PubMed: 22037378]

(32). Wagner FF; Benajiba L; Campbell AJ; Weïwer M; Sacher JR; Gale JP; Ross L; Puissant A; Alexe G; Conway A; Back M; Pikman Y; Galinsky I; DeAngelo DJ; Stone RM; Kaya T; Shi X; Robers MB; Machleidt T; Wilkinson J; Hermine O; Kung A; Stein AJ; Lakshminarasimhan D; Hemann MT; Scolnick E; Zhang Y-L; Pan JQ; Stegmaier K; Holson EB Exploiting an Asp-Glu "switch" in glycogen synthase kinase 3 to design paralog-selective inhibitors for use in acute myeloid leukemia. Sci. Transl. Med 2018, 10, No. eaam8460. [PubMed: 29515000]

(33). Sharma K; Schmitt S; Bergner CG; Tyanova S; Kannaiyan N; Manrique-Hoyos N; Kongi K; Cantuti L; Hanisch U-K; Philips M-A; Rossner MJ; Mann M; Simons M Cell type- and brain region-resolved mouse brain proteome. Nat. Neurosci 2015, 18, 1819-1831. [PubMed: 26523646]

(34). Roy M; Sorokina O; Skene N; Simonnet C; Mazzo F; Zwart R; Sher E; Smith C; Armstrong JD; Grant SGN Proteomic analysis of postsynaptic proteins in regions of the human neocortex. Nat. Neurosci 2018, 21, 130-138. [PubMed: 29203896] 
(35). Shen W; Taylor B; Jin Q; Nguyen-Tran V; Meeusen S; Zhang Y-Q; Kamireddy A; Swafford A; Powers AF; Walker J; Lamb J; Bursalaya B; DiDonato M; Harb G; Qiu M; Filippi CM; Deaton L; Turk CN; Suarez-Pinzon WL; Liu Y; Hao X; Mo T; Yan S; Li J; Herman AE; Hering BJ; Wu T; Martin Seidel H; McNamara P; Glynne R; Laffitte B Inhibition of DYRK1A and GSK3B induces human $\beta$-cell proliferation. Nat. Commun 2015, 6, 8372. [PubMed: 26496802]

(36). Bartoschik T; Maschberger M; Feoli A; André T; Baaske P; Duhr S; Breitsprecher D Microscale Thermophoresis in Drug Discovery Applied Biophysics for Drug Discovery; Wiley, 2017; pp 7392.

(37). Stamos JL; Chu ML; Enos MD; Shah N; Weis WI Structural basis of GSK-3 inhibition by Nterminal phosphorylation and by the Wnt receptor LRP6. eLife 2014, 18, No. e01998.

(38). Cheng C; Fass DM; Folz-Donahue K; MacDonald ME; Haggarty SJ Highly Expandable Human iPS Cell-Derived Neural Progenitor Cells (NPC) and Neurons for Central Nervous System Disease Modeling and High-Throughput Screening. Curr. Protoc. Hum. Genet 2017, 92, 21.8.21 DOI: $10.1002 /$ cphg.33

(39). Zhao WN; Haggerty SJ Discovery of Functional Lithium Mimetics Affecting CRMP2 Phosphorylation in Human iPSC-Derived Neurons. Unpublished results, 2018.

(40). Yoshimura T; Kawano Y; Arimura N; Kawabata S; Kikuchi A; Kaibuchi K GSK-3 $\beta$ Regulates Phosphorylation of CRMP-2 and Neuronal Polarity. Cell 2005, 120, 137-149. [PubMed: 15652488]

(41). Yamashita N; Ohshima T; Nakamura F; Kolattukudy P; Honnorat J; Mikoshiba K; Goshima Y Phosphorylation of CRMP2 (collapsin response mediator protein 2) is involved in proper dendritic field organization. J. Neurosci 2012, 32, 1360-1365. [PubMed: 22279220]

(42). Kim W-Y; Wang X; Wu Y; Doble BW; Patel S; Woodgett JR; Snider WD GSK-3 is a master regulator of neural progenitor homeostasis. Nat. Neurosci 2009, 12, 1390-1397. [PubMed: 19801986]

(43). Zhao W-N; Cheng C; Theriault KM; Sheridan SD; Tsai L-H; Haggarty SJ A High-Throughput Screen for Wnt $/ \beta$-Catenin Signaling Pathway Modulators in Human iPSC-Derived Neural Progenitors. J. Biomol. Screen 2012, 17, 1252-1263. [PubMed: 22923789]

(44). Sasaki A; Shoji M; Harigaya Y; Kawarabayashi T; Ikeda M; Naito M; Matsubara E; Abe K; Nakazato Y Amyloid cored plaques in Tg2576 transgenic mice are characterized by giant plaques, slightly activated microglia, and the lack of paired helical filament-typed, dystrophic neurites. Virchows Arch. 2002, 441, 358-367. [PubMed: 12404061]

(45). Bero AW; Yan P; Roh JH; Cirrito JR; Stewart FR; Raichle ME; Lee J-M; Holtzman DM Neuronal activity regulates the regional vulnerability to amyloid- $\beta$ deposition. Nat. Neurosci 2011, 14, 750-756. [PubMed: 21532579]

(46). Bernard-Gauthier V; Bailey J; Berke S; Schirrmacher R Recent advances in the development and application of radiolabeled kinase inhibitors for PET imaging. Molecules 2015, 20, 2200022027. [PubMed: 26690113]

(47). Ferguson FM; Gray NS Kinase inhibitors: the road ahead. Nat. Rev. Drug Discovery 2018, 17 , 353-377. [PubMed: 29545548]

(48). Zhang L; Villalobos A; Beck EM; Bocan T; Chappie TA; Chen L; Grimwood S; Heck SD; Helal CJ; Hou X; Humphrey JM; Lu J; Skaddan MB; McCarthy TJ; Verhoest PR; Wager TT; Zasadny K Design and selection parameters to accelerate the discovery of novel central nervous system positron emission tomography (PET) ligands and their application in the development of a novel phosphodiesterase 2A PET ligand. J. Med. Chem 2013, 56, 4568-4579. [PubMed: 23651455]

(49). Englebienne P; Moitessier N Docking ligands into flexible and solvated macromolecules. 5. Force-field-based prediction of binding affinities of ligands to proteins. J. Chem. Inf. Model 2009, 49, 2564-2571. [PubMed: 19928836]

(50). Therrien E; Englebienne P; Arrowsmith AG; Mendoza-Sanchez R; Corbeil CR; Weill N; Campagna-Slater V; Moitessier N Integrating Medicinal Chemistry, Organic/Combinatorial Chemistry, and Computational Chemistry for the Discovery of Selective Estrogen Receptor Modulators with Forecaster, a Novel Platform for Drug Discovery. J. Chem. Inf. Model 2012, 52, 210-224. [PubMed: 22133077] 
(51). Corbeil CR; Englebienne P; Yannopoulos CG; Chan L; Das SK; Bilimoria D; L'Heureux L; Moitessier N Docking ligands into flexible and solvated macromolecules. 2. Development and application of FITTED 1.5 to the virtual screening of potential HCV polymerase inhibitors. J. Chem. Inf. Model 2008, 48, 902-909. [PubMed: 18341269] 
A

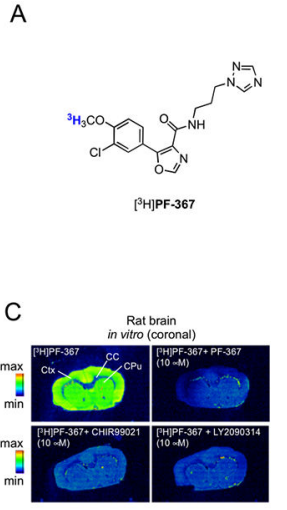

B

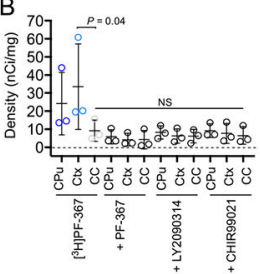

$\mathrm{D}$

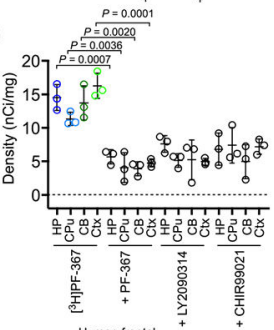

E
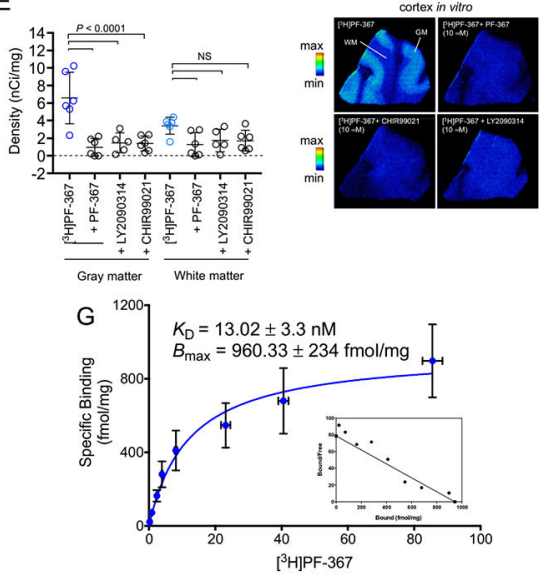

$\mathrm{H}$

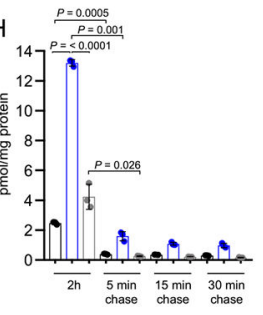

Figure 1.

$\left[{ }^{3} \mathrm{H}\right] \mathrm{PF}-04802367\left(\left[{ }^{3} \mathrm{H}\right] \mathbf{1}\right)$ displays high specific and selective binding to GSK-3 $\alpha / \beta$ in cells and mammalian brains in vitro. (A) Chemical structure of $\left[{ }^{3} \mathrm{H}\right] 1$. (B) Quantification for the in vitro autoradiography of $\left[{ }^{3} \mathrm{H}\right] \mathbf{1}$ in the normal rat brain (20 $\mu \mathrm{m}$ sections, $n=3$ ) under baseline, homologous blocking (nonradioactive 1, $10 \mu \mathrm{M}$ ), and heterologous (LY2090314, $10 \mu \mathrm{M}$ or CHIR99021, $10 \mu \mathrm{M}$ ) conditions. (C) In vitro autoradiography images of $\left[{ }^{3} \mathrm{H}\right] \mathbf{1}$ normal rat brain (coronal sections) under baseline, homologous blocking (nonradioactive 1, $10 \mu \mathrm{M}$ ), and heterologous (LY2090314, $10 \mu \mathrm{M}$ or CHIR99021, $10 \mu \mathrm{M}$ ) conditions. (D) Quantification for the in vitro autoradiography of $\left[{ }^{3} \mathrm{H}\right] \mathbf{1}$ in the normal marmoset brain (20 $\mu \mathrm{m}$ sections, $n=3$ ) under baseline, homologous blocking (nonradioactive $1,10 \mu \mathrm{M}$ ), and heterologous (LY2090314, $10 \mu \mathrm{M}$ or CHIR99021, $10 \mu \mathrm{M}$ ) conditions. (E) Quantification for the in vitro autoradiography of $\left[{ }^{3} \mathrm{H}\right] \mathbf{1}$ in the normal human brain $(20 \mu \mathrm{m}$ sections, frontal cortex, $n=6$ ) under baseline, homologous blocking (nonradioactive 1, $10 \mu \mathrm{M}$ ), and 
heterologous (LY2090314, $10 \mu \mathrm{M}$ or CHIR99021, $10 \mu \mathrm{M}$ ) conditions. (F) In vitro autoradiography images of $\left[{ }^{3} \mathrm{H}\right] \mathbf{1}$ normal human brain under baseline, homologous blocking (nonradioactive 1, $10 \mu \mathrm{M}$ ), and heterologous (LY2090314, $10 \mu \mathrm{M}$ or CHIR99021, $10 \mu \mathrm{M}$ ) conditions. (G) Saturation $\left[{ }^{3} \mathrm{H}\right] \mathbf{1}$ binding curves, Scatchart plot, and $B_{\max }$ determination using the homogenate mouse brain tissue. (H,I) Cell binding assays with $\left[{ }^{3} \mathrm{H}\right] \mathbf{1}$ in HEK-293T P301S hTau cells, overexpressing GSK-3 $\alpha / \beta$ and GSK-3 $\alpha / \beta$ knockdown (shRNA). $\left[{ }^{3} \mathrm{H}\right] \mathbf{1}$ binding under $2 \mathrm{~h}$ baseline and following timecourse chase experiments. $(\mathrm{J})$ Western blot analysis of control, GSK-3 $\alpha / \beta$-overexpressing, and GSK-3 $\alpha / \beta$ shRNA cells used for $\left[{ }^{3} \mathrm{H}\right] 1$-binding studies. Data show mean values \pm standard deviation (SD). Statistical significance was calculated one-way ANOVA followed by Dunnett's test (B,D,E,H). 
A

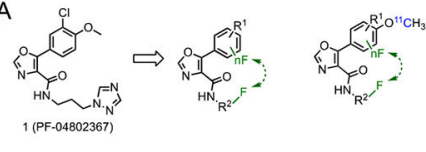

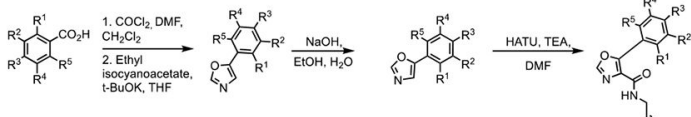

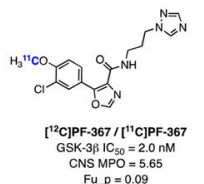

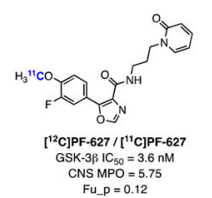

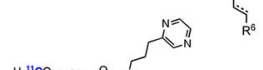

C

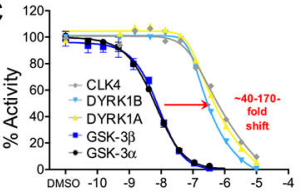

Log [PF-618] (M)
D
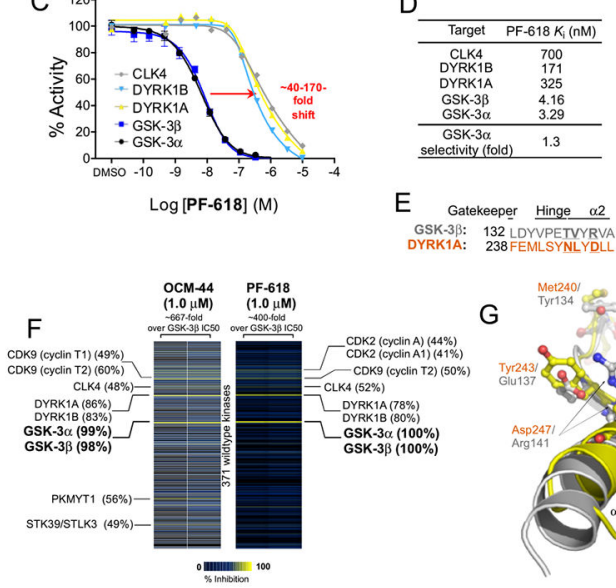

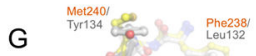

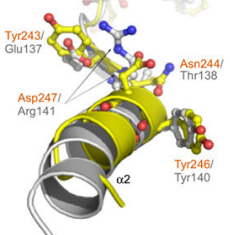
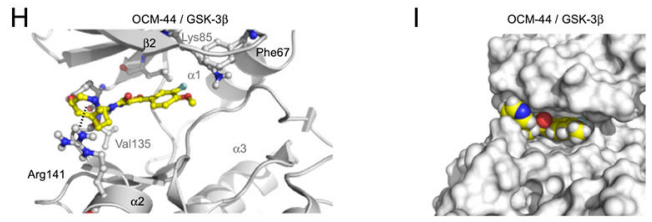

Figure 2.

Evolution and in vitro pharmacological characterization of a highly potent and selective oxazole-4-carboxamide-based GSK-3 inhibitor series. (A) Generalized strategy to synthesize arrays of carboxamide-based GSK-3 inhibitors, as well as structures and properties of selected GSK-3 inhibitors (for details on chemical synthesis, see Schemes S1-S4). IC 50 values determined using an ADP-Glo kinase assay $(n=2)$. (B) Heat map representing key properties [ $\mathrm{IC}_{50}$, central nervous system multiparameter optimization (CNS MPO), and Fu_p] of oxazole-4-carboxamide-based GSK-3 inhibitors including a comparison with previously described GSK-3 PET radiotracers (for structures and detailed parameter values, see Tables S1-S4). IC 50 values determined using an ADP-GloTM kinase assay $(n=2)$. $(\mathrm{C}, \mathrm{D})$ The dose-response curves and binding affinities of the representative inhibitor 28 versus GSK-3 $a$, GSK- $3 \beta(n=3)$, and main kinases off targets DYRK1A, DRYK1B, and CLK4 $(n=1)$ demonstrate high selectivity for GSK-3a/ $\beta$ (radiometric filtration binding assay, $[\mathrm{ATP}]=K_{\mathrm{m}} \mathrm{ATP}$, data show mean values $\pm \mathrm{SD}$ ). (E) Hinge/ $a 2$ region sequence alignment of GSK-3 $\beta$ and the main kinase secondary target DYRK1A. (F) Comprehensive kinase selectivity profile of representative inhibitor 44 and 28 against 371 kinases (kinases are ordered alphabetically, and the activity is relative to control at $1.0 \mu \mathrm{M}$ inhibitor concentration, $n=2$, [ $\left.\gamma \_{ }^{33} \mathrm{P}\right]$ ATP-based enzymatic assay performed by Reaction Biology, for detailed data see Table S5). (G) Overlay of the hinge/ $a 2$ region of GSK-3 $\beta$ (gray, PDB ID code 5K5N) and DYRK1A (yellow, PDB ID code 3ANR). (H) Predicted detailed interaction of $\mathbf{4 4}$ (yellow sticks) with GSK-3 $\beta$ (gray cartoon, PDB ID code $5 \mathrm{~K} 5 \mathrm{~N}$ ). The $\boldsymbol{\pi}$ cation interaction is depicted as a black dash line. (I) Alternate view of the binding mode of 44 (yellow, space fill model) with GSK-3 $\beta$ (surface rendering, PDB ID code 5K5N). CNS MPO; Fu_p, in silico calculated unbound fraction in plasma. 

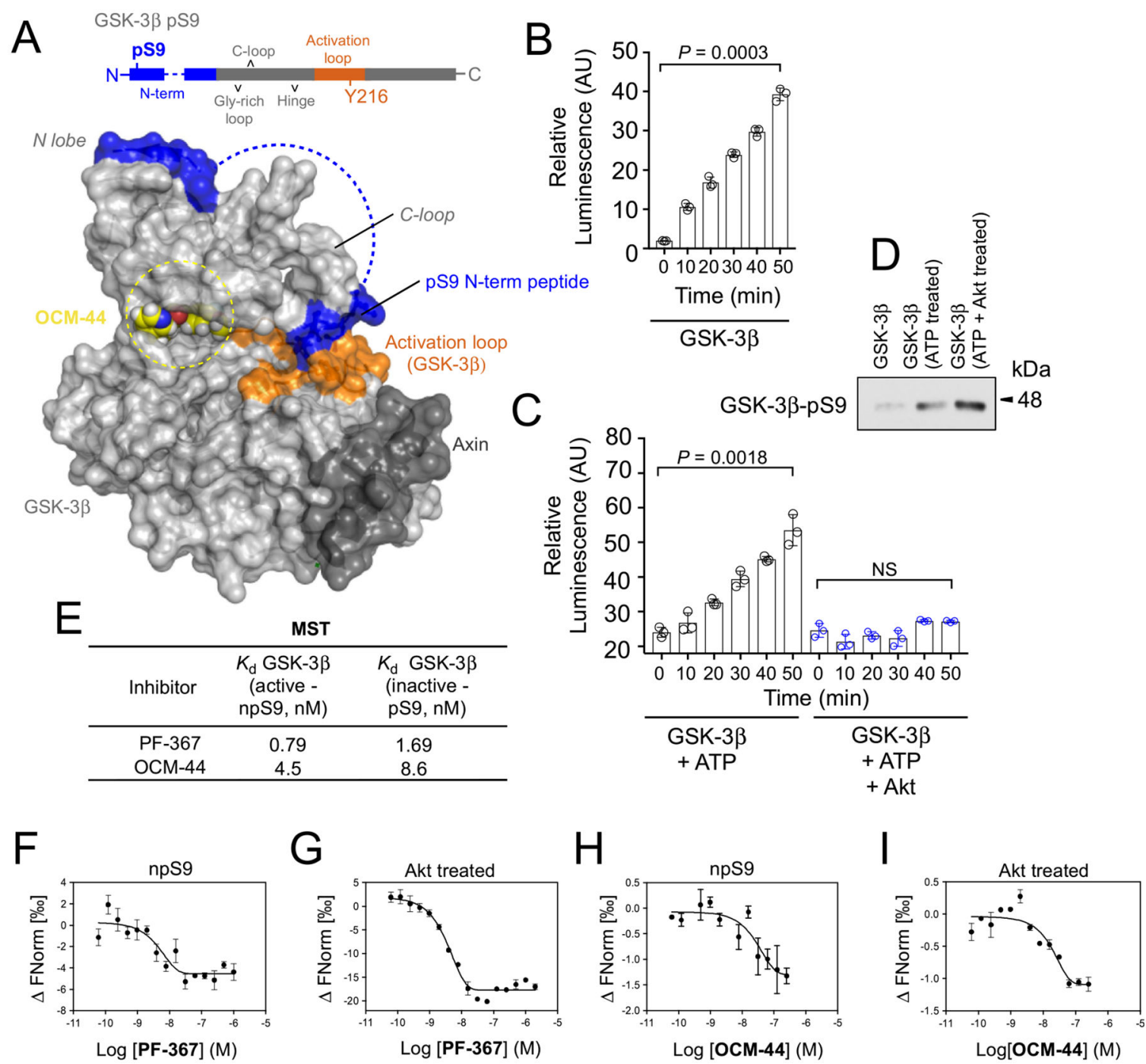

Figure 3.

Oxazole-4-carboxamide-based probes engage both active npS9 and catalytically inactive pS9 GSK-3 $\beta$. (A) Structural basis for the recognition of inhibitors by pS9-GSK-3 $\beta$ (44 is depicted as a representative inhibitor, yellow, space fill model). The predicted binding mode illustrates the distal positioning of $\mathbf{4 4}$ from the activation loop and pS9 auto-inhibitory $\mathrm{N}$ terminal peptide (blue surface) and the negligible ATP-binding site rearrangement upon binding of the $\mathrm{pS} 9$ auto-inhibitory N-terminal peptide of GSK-3 $\beta$ (gray surface, PDB ID code 4NM3). (B) Timecourse analysis showing GSK-3 $\beta$ activity in the presence of low ATP content and GSP2 (ADP-Glo-based kinase assay, $n=3$ ). (C) Timecourse analysis showing GSK- $3 \beta$ activity in the presence of high ATP content and high ATP content combined with AKT (ADP-Glo-based kinase assay, $n=3$ ). (D) Western blot analysis of enzymatic reaction contents (control, ATP treated and ATP + AKT treated). (E) Binding affinities of $\mathbf{1}$ and $\mathbf{4 4}$ measured by MST. (F,G) MST dose-response curves for the binding interaction of between 1 with active npS9-GSK-3 $\beta$ and pS9-GSK-3 $\beta(n=2)$. (H,I) MST dose-response curves for the binding interaction of $\mathbf{4 4}$ with active npS9-GSK-3 $\beta$ and pS9-GSK-3 $\beta(n=2)$. Data show mean values \pm SD. Statistical significance was calculated with paired $t$-test $(B, C)$. 

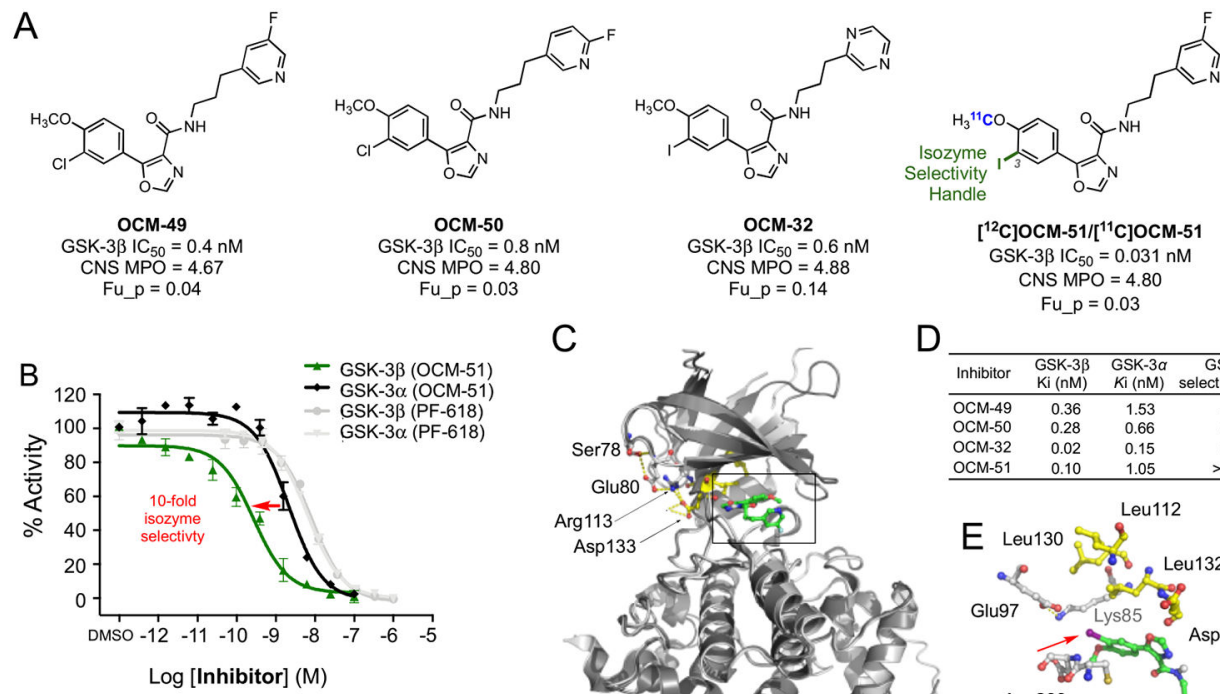

C

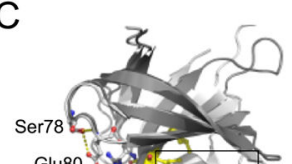

D
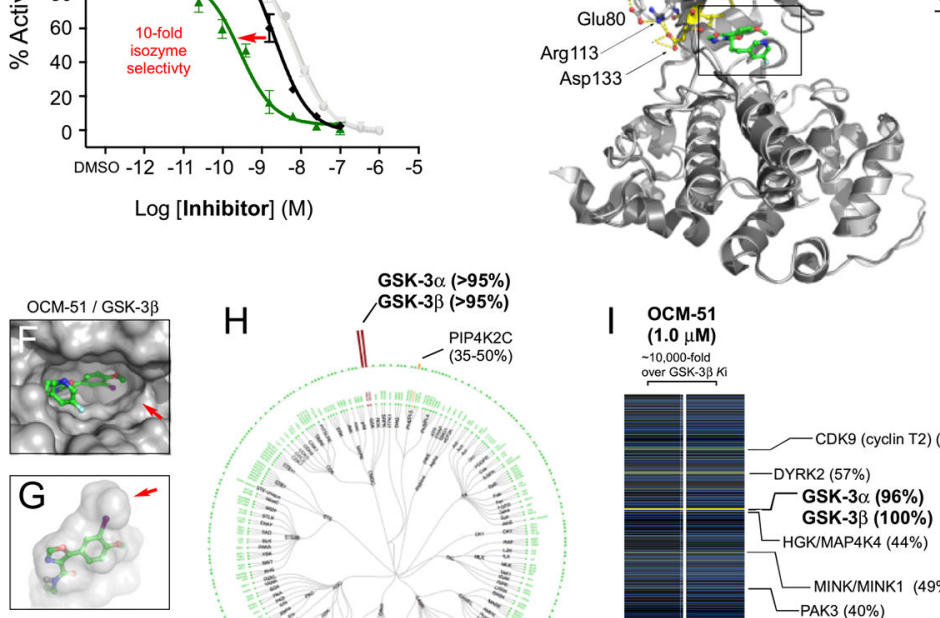

| OCM-51 $(1.0 \mu \mathrm{M})$

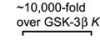
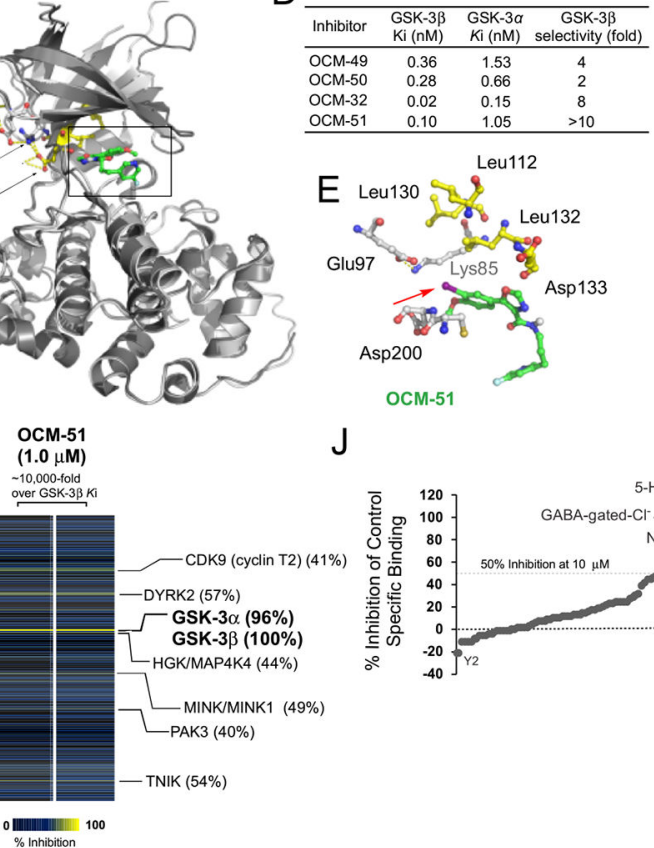

Leu112

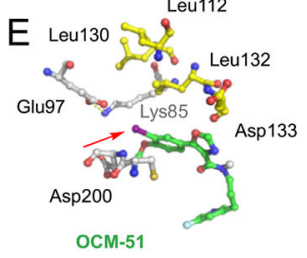

$\mathrm{J}$

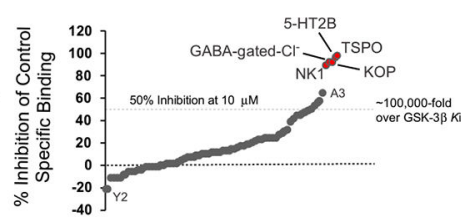

$\mathrm{K}$

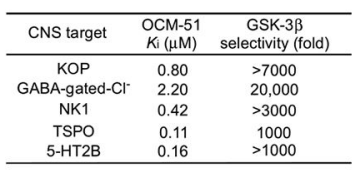

L

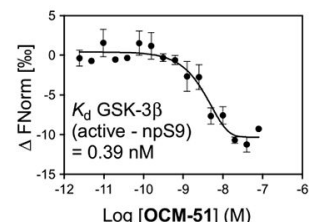

M

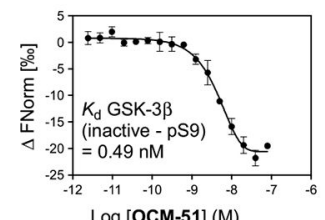

Figure 4.

Structure-guided design and characterization of the GSK-3 $\beta$ isozyme-selective inhibitors $5 \mathbf{5 1}$. (A) Structures and properties of selected GSK-3 inhibitors leading to $\mathbf{5 1}$ (for details on chemical synthesis, see Schemes S1-S4). IC 50 values determined using an ADP-Glo kinase assay $(n=2)$. (B) Dose-response curves of 51 versus GSK-3 $a$ and GSK- $3 \beta(n=3)$

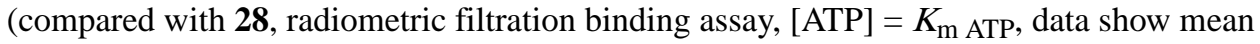
values \pm SD). (C) Predicted binding mode of 51 (green sticks) with GSK-3 $\beta$ (gray cartoon, PDB ID code $5 \mathrm{~K} 5 \mathrm{~N}$ and 5KPK). Highlight on the interaction network associated with Asp133. Hydrogen bonds are depicted as a yellow dash lines. (D) Binding affinity measurements of inhibitors versus GSK-3 $a$ and GSK-3 $\beta(n=3)$. (E-G) Alternate views of the predicted contact between $\mathbf{5 1}$ and the selectivity pocket of GSK-3 $\beta$. (H) Kinativ data from mice brain lysates showing the selective engagement of the representative inhibitor 49 $(1.0 \mu \mathrm{M})$ with GSK-3 ( $n=2$, for detailed data, see Table S5). (I) Comprehensive kinase 
selectivity profile of representative inhibitor $\mathbf{5 1}$ against 371 kinases (kinases are ordered alphabetically, and the activity is relative to control at $1.0 \mu \mathrm{M}$ inhibitor concentration, $n=2$, $\left[\gamma^{3}{ }^{33} \mathrm{P}\right]$ ATP-based enzymatic assay performed by Reaction Biology, for detailed data see Table S9). (J) Selectivity profile of $\mathbf{5 1}(10.0 \mu \mathrm{M})$ against a CNS target panel $(n=2,80$ targets). (K) Binding affinity of $\mathbf{5 1}$ against the five most inhibited CNS targets at a concentration 100000-fold over the $K_{\mathrm{i}}$ of $\mathbf{5 1}$ for GSK-3 $\beta$. (L) MST dose-response curve and binding affinity for the interaction between $\mathbf{5 1}$ with active npS9-GSK- $3 \beta$ and pS9-GSK-3 $\beta$ $(n=2)$. (M) MST dose-response curve and binding affinity for the interaction between $\mathbf{5 1}$ with active npS9-GSK-3 $\beta$ and pS9-GSK- $3 \beta(n=2)$. Data show mean values \pm SD. 

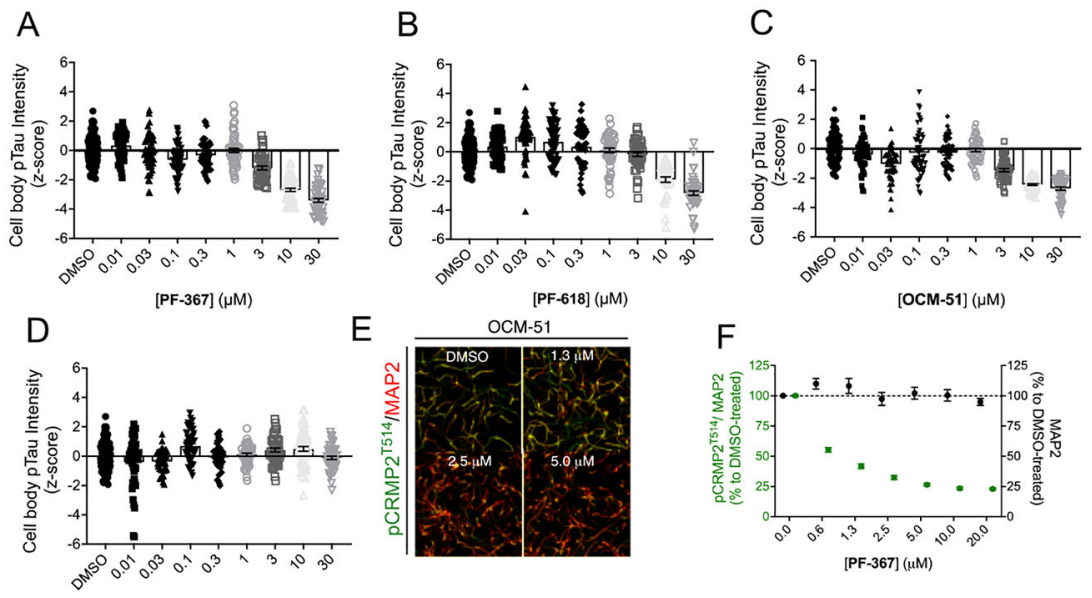

[OCM-51] ( $\mu \mathrm{M})$
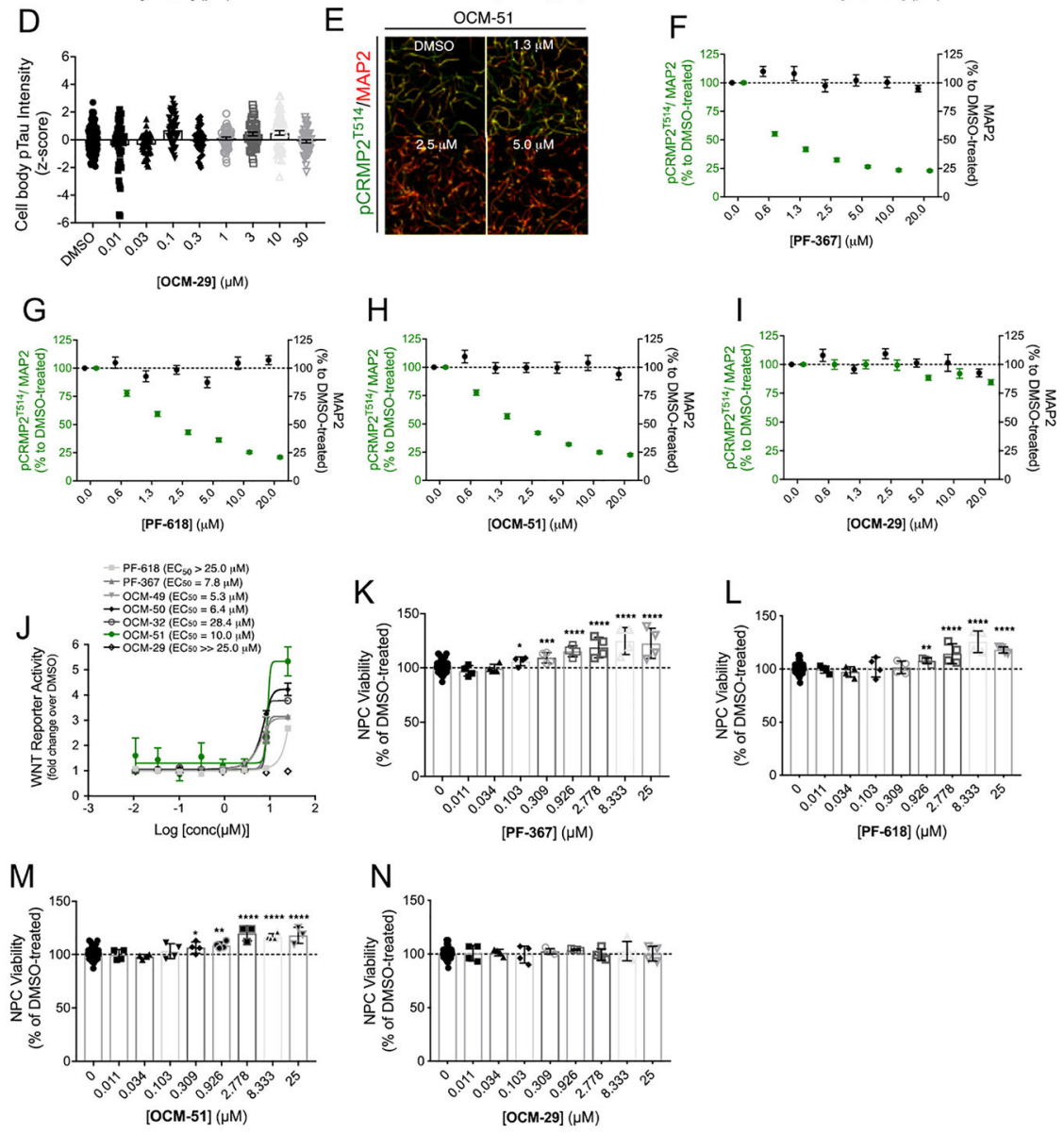

Figure 5.

Selective oxazole-4-carboxamide GSK-3 inhibitors modulate both pTau and pCRMP2 ${ }^{\mathrm{T} 514}$ and display favorable therapeutic index against $\mathrm{Wnt} / \beta$-catenin signaling in human neuronal cells. (A-D) Dose-dependent effect of selected compounds on decreasing phosphorylated tau in human neurons. $20 \mathrm{~h}$ treatment with $\mathbf{1 , 2 8 , 5 1}$, but not 29 dose-dependently decreased mean pTau (PHF-1) intensity in neuronal cell bodies. Each bar is the mean values \pm SEM of 14-21 fields/well. Each dose of each compound was tested in 1 well per plate. Data from 3 plates in total are shown in the figure. $(\mathrm{E}-\mathrm{H})$ High-content imaging assays using human neurons showed that representative inhibitors $(\mathbf{1}, \mathbf{2 8}, \mathbf{5 1})$ reduced CRMP2 phosphorylation in a dose-dependent manner whereas $\mathbf{2 9}$ did not. CRMP2 is a direct substrate of GSK-3 $\beta$. The relative level of $\mathrm{pCRMP} 2^{\mathrm{T} 514}$ intensity was reported as the percentage of the intensity for dimethylsulfoxide (DMSO)-treated (100\%) ( $n=3$ biological replicates, $n=18$ images for analysis. Data show mean values \pm SEM). Relative levels of dendritic marker MAP2 
were not affected by the compound treatments, indicating the health of neurons. (I) Representative images of $\mathbf{5 1}$ treatment on $\mathrm{pCRMP2} 2^{\mathrm{T} 514}$ intensity (green) versus MAP2 intensity (red) illustrate the dose-dependent $\mathrm{pCRMP} 2^{\mathrm{T} 514}$ intensity reduction. (J-M) Proneurogenic potentials measured as NPC proliferation were detected for active oxazole-4carboxamide GSK-3 inhibitors. Graphs show dose-dependent increases from PF-367, PF-618, and $\mathbf{5 1}$ but not 29. NPC proliferation is expressed as the percentage of DMSOtreated $(100 \%)$. $(n=4$. Error bars display SEM. Unpaired $t$-test. $* 0.01 \leq p<0.05, * * 0.001 \leq$ $p<0.01, * * * 0.0001 \leq p<0.001, * * * * p<0.0001$.) (N) Active oxazole-4-carboxamide GSK-3 inhibitors enhanced Wnt/ $\beta$-catenin signaling in human NPCs. Dose-response curves of representative inhibitors $\left(\mathbf{1}, \mathbf{2 8}, \mathbf{5 1}\right.$, and inactive 29) with calculated $\mathrm{EC}_{50} \mathrm{~s}$ are shown. $n=$ 4. Data show mean values \pm SEM. 

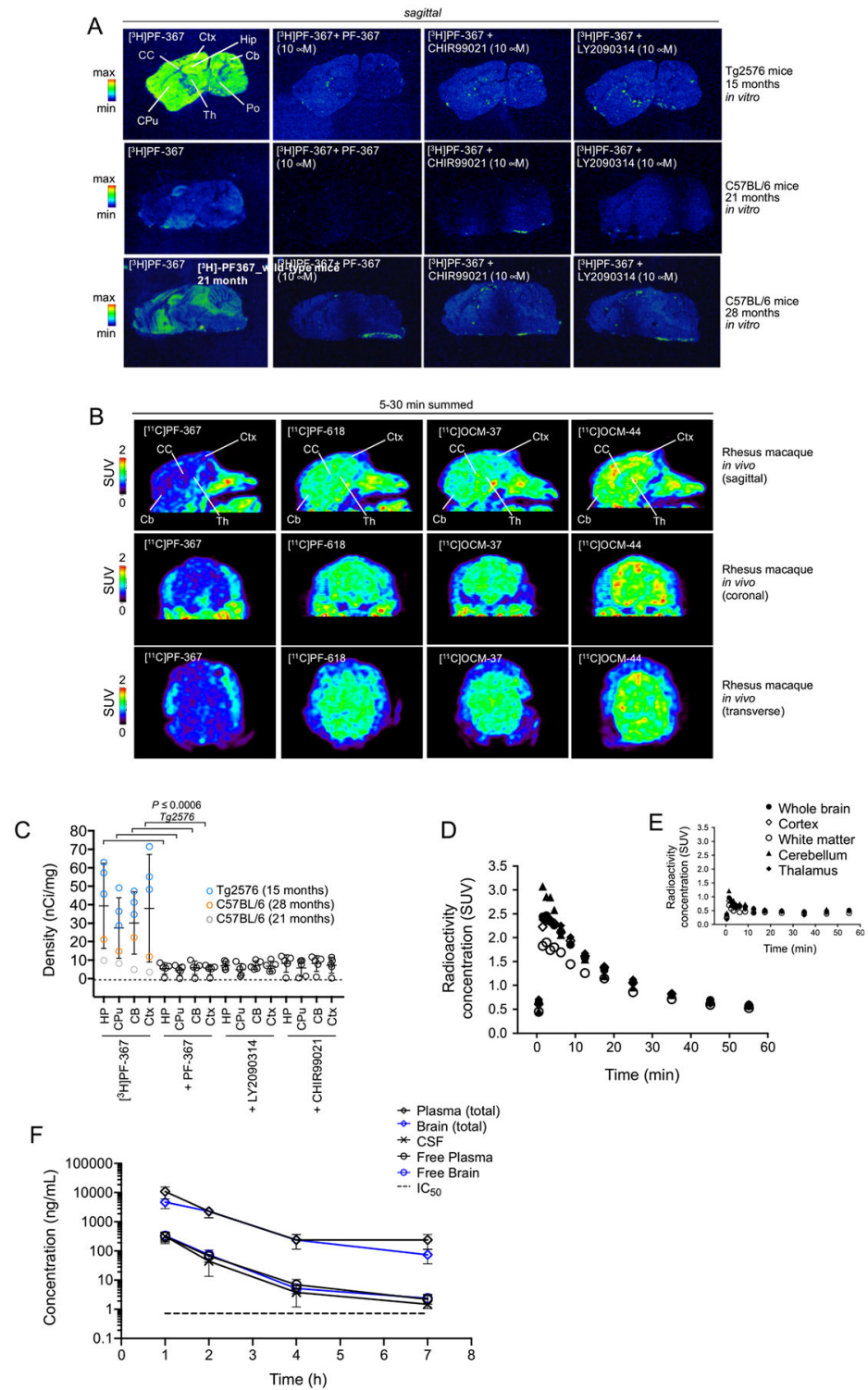

Figure 6.

Oxazole-4-carboxamide-based probes display robust brain exposure properties in vivo and differential target engagement in a mouse model of AD in vitro. (A) In vitro autoradiography images of $\left[{ }^{3} \mathrm{H}\right] \mathbf{1} \mathrm{Tg} 2576$ and C57BL/6 mice under baseline, homologous blocking (nonradioactive 1, $10 \mu \mathrm{M}$ ), and heterologous (LY2090314, $10 \mu \mathrm{M}$ or CHIR99021, $10 \mu \mathrm{M}$ ) conditions. (B) Summed (5 to $30 \mathrm{~min}$ ) PET images showing the distribution of $\left[{ }^{11} \mathrm{C}\right] \mathbf{2 8}$, $\left[{ }^{11} \mathrm{C}\right] \mathbf{3 7}$, and $\left[{ }^{11} \mathrm{C}\right] \mathbf{4 4}$ in comparison with $\left[{ }^{11} \mathrm{C}\right] \mathbf{1}$ in the rhesus monkey brain, following microdose injections. Image intensity displayed as 0-2 SUV scale. (C) Quantification for the in vitro autoradiography of $\left[{ }^{3} \mathrm{H}\right] \mathbf{1}$ in $\mathrm{Tg} 2576(20 \mu \mathrm{m}$ sections, $n=3)$ and C57BL/6 (20 $\mu \mathrm{m}$ sections, $n=2$ ) mice brains under baseline, homologous blocking (nonradioactive 1, 10 $\mu \mathrm{M}$ ), and heterologous (LY2090314, $10 \mu \mathrm{M}$ or CHIR99021, $10 \mu \mathrm{M}$ ) conditions. (D) Representative rhesus monkey regional brain time-activity curves (TACs) after the intravenous injection of $\left[{ }^{11} \mathrm{C}\right] \mathbf{4 4}$. (E) Representative rhesus monkey regional brain time- 
activity curves (TACs) after intravenous injection of $\left[{ }^{11} \mathrm{C}\right] \mathbf{1}$. (F) Mice exposure of $4 \mathbf{4}$ (C57BL/6 mice, $50 \mathrm{mg} / \mathrm{kg}$, single dose PO, $n=3$ ). Data show mean values $\pm \mathrm{SD}$. 